\title{
ION CYCLOTRON AND SPIN-FLIP EMISSIONS FROM FUSION PRODUCTS IN TOKAMAKS
}

\author{
V. Arunasalam, G. J. Greene, and K. M. Young PPPL--2875 \\ Princeton University, Plasma Physics Laboratory \\ DE93 007935 \\ Princeton, N.J. 08543
}

\begin{abstract}
Power emission by fusion products (i.e., protons and/or alpha particles) of tolcamak plasmas in their ion cyclotron range of frequencies (ICRF) and at their spin-flip resonance frequency is calculated for some specific model fusion product velocity-space distribution functions. The background plasma of say deuterium (D) is assumed to be in equilibrium (or nosequilibrium) with a Maxwellian distribution both for the electrons and ions (with a possible vemperature anisotropy and trift velocity for the $\mathrm{D}$ ions). The fusion product velocity distributiors analyzed here are: (1) A monoenergetic velocity space ring distribution. (2) A monoenergetic isouropic velocity space spherical shell distribution.(3) An anisotropic Maxwellian distribution with $T_{\perp} \approx T_{\|}$and with appreciable drift velocity along the confining magnetic field. Single "dressed" test particle spontaneous emission calculations are presented first and the radiation temperature for ion cyclotron emission (ICE) is analyzed both for black-body emission and nonequilibrium conditions. Thresholds for instability and overstability (iLe., negative rediation temperature) conditions are then examined and quasilinear and nonlinear theories of the eloctromagnetic ion cyclotron modes are discussed. Distinctions between "kinetic or causal instabilities" and "hydrodynamic instabilities" are drawn and some numerical estimates are presented for typical tokamak parameters. Semiquantitative remartas are offered on wave accessibility, mode conversion, and parametric decay instabilities as possible mochanisms for spatially localized ICE. Calculations are carried out both for $k_{11}=0$ for $k_{1} \neq 0$. The effects of the temperature anisotropy (i.e., $T_{\perp} \neq T_{11}$ ) and large drift velocities in the parallel direction are also examined. Finally, proton spin-flip resonance emission and absorption calculations are also presented both for thermal equilibrium conditions and for an "inverted" population of states. The method of analysis is the familiar "master equation approach" of nonequilibrium quantum statistical mechanics, based on the Einstein A and B coefficients and the principle of detailed balance. Reasonably good agreement is obrained between theory and experiment.
\end{abstract}

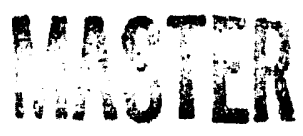




\section{INTRODUCTION}

Quantitative measurements of ion cyclotron emission (ICE) from energetic ions produced by fusion reactions or neutral beam injection promises to be a useful diagnostic on large tokamak devices that are entering the reactor regime of operation. Indeed, the mere qualitative observation of thermal or superthermal radiation in the ion cyclotron range of frequencies (ICRF) provides, in principle, a method for detecting and studying these energetic charged particles in magnetic fusion experiments. Historically, such ICE was first reported by the TFR Groupl during neutral beam injection experiments. Somewhat similar ICE was also observed from neutral beam injected PDX discharges. ${ }^{2}$ However, the first observation of ICE from fusion products came from JET plasmas. 3.4 Subsequently, ICE from fusion products has been reported from TFTR discharges. 5 These fusion pruducts of the primary DD reaction are given by

$$
D+D=\left\{\begin{array}{l}
{ }^{3} \mathrm{He}(0.82 \mathrm{MeV})+\mathrm{n}(2.5 \mathrm{MeV}) \\
\mathrm{T}(1.0 \mathrm{MeV})+\mathrm{p}(3.0 \mathrm{MeV})
\end{array},\right.
$$

and the secondary reactions are given by

$$
\begin{aligned}
& 3_{\mathrm{He}}+\mathrm{D}=\mathrm{p}(14.7 \mathrm{MeV})+{ }^{4} \mathrm{He}(3.7 \mathrm{MeV}), \\
& T+\mathrm{D}={ }^{4} \mathrm{He}(3.6 \mathrm{MeV})+\mathrm{n}(14.7 \mathrm{MeV})
\end{aligned}
$$

There are experimental differences, both qualitative and quantitative, between the features of ICE observed in different tokamaks. These variations may be related to the different experimental configurations employed. The JET experiment uses an ICRF heating antenna as a receiver and this antenna is located at the outer midplane and has significant poloidal and toroidal extent. The TFTR and FDX experiments have used small magnetic probes to detect ICE. These probes have been located both at the outer (TFTR) and inner (PDX) midplane and, more recently, near the top and bottom of the vacuum vessel (TFTR). In general, results from PDX and TFTR experiments are similar to each other but differ in certain respects from the JET results.

Experiments during neutral beam injection in PDX, TFR, JET, and TFTR $1-5$ have all revealed an emission spectrum that includes intense (i.e., $10^{4}$ times the background level), regularly spaced peaks whose narrowness $\left(\Delta \omega / \omega \ll a_{p} / R_{p}\right)$ implies a spatially localized origin (since the ion cyclotron frequency for an ionic species of charge $q_{B}$ and mass $M_{B}$ in the tokamak $B$-field is $\left.\omega_{c B}=q_{B} B / M_{B} c \propto R^{-1}\right)$. Here, $R_{p}$ and $a_{p}$ are the plasma major and minor radius, respectively. Emission is seen at approximately $n \omega_{C B-E}$, where $n$ is a positive integer and $\omega_{C B-E}$ is the cyclotron 
frequency for the ionic species $B$, evaluated at the outer (low-field) plasma edge on the midplane (i.e., at $\mathbf{R}=\mathbf{R}_{\mathrm{p}}+\mathrm{a}_{\mathrm{p}}$ ). In PDX and TFTR with different injected and background species, $B$ is determined to be the injected species; however, explanation of data LFom JET experiments requires $B$ to be background species. Reported widths of the peaks also differ: in PDX and TFTR, $\Delta \omega / \omega$ is typically of the order of 0.005 ; in the JET Experiment, $\Delta \omega / \omega$ is of the order of 0.05 .

Ion cyclotron emission from ohmic discharges in deuterium has been observed in JET 3,4 and TFTR.5 In the JET experiment, this emission consists of a series of harmonically related peaks, as in the beam injection case, occuring near n $\omega_{c d-E}$ (where $\omega_{c d-E}$ is the deuteron cyclotron frequency at the outer low-field plasma edge on the midplane). Note that $\omega_{c d-E}=\omega_{c \alpha-E}$, where $\omega_{c} \alpha-E$ is the corresponding alpha particle cyclotron frequency at the same location. Typical peak widths are broader than in the beam injection case: $\Delta \omega / \omega=0.1$. In contrast, ohmic ICE in the TFTR experiment consists of a sequence of peaks at frequencies $(n+1 / 2) \omega \mathrm{cp}-\mathrm{E}$, with peak widths also of the order of $\Delta \omega / \omega=0.1$ (i.e., broader than the beam injection case by a factor of about 20). Here, $\omega_{c p-E}=2 \omega_{c d-E}$ is the proton cyclotron frequency at the outer low-field plasma edge on the midplane. In addition, both experiments show considerably less intense ICE in the absence of beam injection.

Recent observation of inverted ICE sawtooth oscillations ${ }^{6}$ which coincide with the arrival of the sawtooth heat pulse in the edge plasma in JET give further support to the edge lucalization of the emission. These experimental observations suggest that the charged fusion products provide the free energy to generate the ICE, and these fusion products are apparently localized in the outer, low B-field side plasma edge region of the tokamak discharges.

In TFTR, an additional feature in the frequency-power spectrum of the ICE signal is observed in high power neutral beam injected discharges. ${ }^{7}$ When deuterium neutral beams are injected into a deuterium background plasma, the main sequence of ICE harmonic peaks (occuring at n $\omega_{c d}-E$ ) is accompanied by a broader, background continuum component of the spectrum that begins around the fifth harmonic of $\omega_{\text {cd-E. }}$. This "background emission" exists roughly over the frequency range for which the proton spin-flip resonance is within the plasma. (Note that the proton $\mathrm{g}$ factor is equal to 5.59, so the fifth harmonic of $\omega_{c d-E}$ is near the frequency at which the proton spin-flip resonance is at the outer plasma edge). A spectrum from TFTR that includes these features is shown in Fig. 1.

The amplitude of a single ICE peak $(n=2)$ in JET was found to be proportional to the measured fusion DD reaction rate (based on $2.5 \mathrm{MeV}$ neutron fluxes) over three orders of magnitude in signal intensity 4 (i.e., 10-12 $\leq$ ICE power $P_{I C E} \leq 10^{-9} \mathrm{~W}$ for $10^{10} \leq$ total DD 
reaction rate $\leq 10^{13} \mathrm{sec}^{-1}$ ), supporting a fusion product origin for the emission. Indeed, very recent measurements in JET during DD and DT experiments have shown a linear correlation between ICE power PICE and the total neutron flux extending over six orders in magnitude ${ }^{4}$ (i.e., $10^{-11} \leq \operatorname{PICE}_{\text {ICE }} \leq 10^{-5} \mathrm{~W}$ for $10^{12} \leq$ total neutron source rate $\leq 10^{18} \mathrm{sec}^{-1}$ ); the best fitting relation is $\mathrm{PICE}_{\mathrm{IC}} \propto$ (neutron flux) $^{0.9}$. Further, by keeping the neutral beam power constant and comparing the $n=2$ PICE from plasmas dorninated by DT fusion reactions with the corresponding plasmas dominated by the DD fusion reactions, these very recent JET experiments showed that the ICE signal is related to fusion products, not beam particles. Also, the ICE intensity is anticorrelated with large amplitude edge-localized modes (ELMs); suggesting that the large ELMs terminate the ICE by expelling the fast ions, thereby temporarily extinguishing the source. In both DD and DT plasmas, the ICE emission lines near $\omega=n \omega_{\text {cd-E }}$ each show a fine structure for $\mathrm{n} \leq 7$, being split into a doublet with $\Delta \omega / \omega=0.06$. For $n>7$, the lines merge into a continuum, possibly related to the "broadband background spin-flip emission" feature observed in TFTR.

In TFTR, however, the amplitude of the $n=2$ ICE peak during beam injection does not follow the neutron flux (as observed within a single shot). However, the broadband background continuum component of the spectrum discussed above (between ICE peaks) does follow the time evolution of the neutron flux, over about 2.5 orders of magnitude, with a delay of about $100 \mathrm{msec}$. This delay was observed to be similar to the growth time for DD fusion products. ${ }^{7}$ In a recent JET experiment, where the background continuum emission was not observed, the signal obtained by integrating the entire recorded ICE spectrum (over many harmonics) had a time evolution that similarly followed the neutron flux with a time delay which is approximately equal to the growth time of the fusion product population.

It is the aim of this paper to examine all the direct emission processes tnat can account quantitatively for the observed emission from the fusion product protons and alpha particles in tokamak plasmas in their ion cyclotron range of frequencies (ICRF) and at the proton spin-flip resonance frequency. We obtain closed form analytic expressions for such direct emissions. These direct emissions include both the single "dressed" test particle spontaneous emission and the collective many-body emission from systems at thermodynamic equilibrium (i.e., black-body emission) and also from unstable nonequilibrium systems (i.e., the cyclotron harmonic and spinflip laser and maser emission). Indirect emission processes such as those due to modetransformation and/or mode-conversion, and those due to the parametric decay instabilities, are discussed only qualitatively. For example, according to $S$ tix 8 one may conceive of the excitation of electrostatic ion Berstein waves (ESIBW) and subsequent mode-conversion into the transverse 
electromagnetic ion cyclotron harmonic waves at the resonance and/or cut-off layer near the lower hybrid frequency $\omega_{\mathrm{LH}}$, where $\omega_{\mathrm{LH}}$ is given by $\omega_{\mathrm{LH}}^{-2}=\left[\left(\omega_{\mathrm{ci}}^{2}+\omega_{\mathrm{pi}}^{2}\right)^{-1}+\left(\omega_{\mathrm{ci}} \omega_{\mathrm{ce}}\right)^{-1}\right]$. Here, $\omega_{\mathrm{ci}}, \omega_{\mathrm{ce}}$, and $\omega_{\mathrm{pi}}$ are the ion cyclotron, electron cyclotron, and the ion plasma frequencies, respectively. Most of the existing mode conversion calculations 8.9 near the lower ( $\left.\omega_{\mathrm{LH}}\right)$ and the upper ( $\left.\omega_{\mathrm{UH}}\right)$ hybrid resonance frequencies are, at best, semiquantitative in nature. Here $\omega_{\mathrm{UH}}^{2}=\omega_{\mathrm{ce}}^{2}+\omega_{\mathrm{pe}}^{2}$ and $\omega_{\mathrm{pe}}$ is the electron plasma frequency. Calculations of the coefficients of transmission and reflection at mode conversion, based on the cortinuity connections at the joints of piecewise solutions of the inhomogeneous plasma-wave equation on either side of the mode conversion layer, are extremely difficult to carry out exactly. However, such a mode-conversion theory has the attractive feature of the spatial localizability of the emission in the close neighborhood of this resonance and/or cut-off layer. Indeed, arguing from Stix's analysis, ${ }^{8}$ Ono has recently suggested 10 such a mode-conversion emission as a possible explanation of the localization of observed ICE on the low B-field side edge of the tokamak plasma.

A somewhat similar anomalous electron cyclotron emission (ECE) of up to 25 harmonics was reported in the early literature by Landauer ${ }^{11}$ and other investigators. 12 The amplitudes of the successive harmonics, although they are decreasing, are of the same order of magnitude. But these amplitudes are several orders of magnitude greater than those calculated for a warm plasma with a Maxwellian distribution of electron velocities. Proposed explanations ${ }^{13}$ of these anomalous ECE results have included sheath phenomena, inhomogeneous electric fields, distorted nonequilibrium velocity distribution functions, coupling of electrostatic to electromagnetic oscillations $\backslash$ ia Stix's theory of mode conversion, 8 and gradients in density and magnetic field. However at present, fully satisfactory theoretical explanations of these anomalous ECE results do not exist. Indeed, the ICE problem under study here is closely similar to the familiar Landauer problem. ${ }^{11}$

With this complexity in mind, we examine ICE and also spin-flip emission problems using the well-known "master equation approach" of nonequilibrium quantum statistical mechanics, based on the Einstein A and B coefficients and the principle of detailed balance. ${ }^{14}$ In Sec. II, we examine the theory of spontaneous emission from "dressed" test particles. Section III addresses the linearized theory of the radiative steady state. In Sec. IV, we derive and outline the necessary and sufficient conditions for radiative instability or overstability. Section V discusses quasilinear and nonlinear theories of the radiative steady state. In Sec. VI, we make some general comments on instabilities relating to the distinctions between the "kinetic or causal instabilities" and the "hydrodynamic instabilities." Section VII offers some numerical estimates for typical tokamak parameters. In all cases of Secs. II-VII, we examine not only the ion cyclotron harmonic emission 
and absorption but also the fusion product protons' spin-flip emission and absorption. Section VIII, offers some brief qualitative and semiquantitative rcmarks on wave accessibility, mode conversion and parametric decay instability. Finally, Sec. IX contains our conclusions and summary. We believe that the rather extensive and comprehensive analysis in conjunction with some discussions of relevant side issues presented here not only will be of pedagogical interest (as it is a particle-orbit analysis based on Einstein $A$ and $B$ coefficients for the fundamental emission and absorption processes) but also will serve as a first step towards a full understanding of the observed ICE and spin-flip emission in tokamaks.

\section{THEORY OF SPONTANEOUS EMISSION}

In this section we first examine the spontaneous cyclotron emission both from the background deuterium plasma ions and the fusion product protons and/or alpha particles, and second we then examine the spontaneous spin-flip resonance emission from protons.

\section{A. Cyclotron Emission}

We consider the cyclotron emission of a particle of charge $q_{i}$ and mass $M_{i}$ in a static magnetic field $B=B i_{z}$. Let $A(m)$ be the Einstein's (quantum mechanical) spontaneous emission probability coefficient for the emission of a photon of frequency $\omega \approx m \omega_{c i}$ and wave vector $k$, where $\omega_{c i}=q_{i} B / M_{i} c$ is the particle's cyclotron frequency. Then, it shown elsewhere ${ }^{15-18}$ that in the the classical limit] $h \mathrm{~A}(\mathrm{~m})$ is given by

$$
\hbar A(m)=\left(\frac{4 \pi^{2} q_{i}^{2}}{L^{3} C \omega}\right)\left[v_{\perp} J_{m}^{\prime}\left(\lambda_{i}\right)\right]^{2} \delta\left(\omega-m \omega_{c i}-k_{\| 1} v_{\|}\right)
$$

for emission of the extraordinary $(\mathrm{X})$ mode near the $\mathrm{m}$-th harmonic, and

$$
h A(m)=\left(\frac{4 \pi^{2} q_{i}^{2}}{L^{3} C \omega}\right)\left\{\left[\frac{\left(\mu^{-1} c \cos \theta-v_{\|}\right)}{\sin \theta}\right] J_{m}\left(\lambda_{i}\right)\right\}^{2} \delta\left(\omega-m \omega_{c i}-k_{\|} v_{\| 1}\right)
$$

for emission of the ordinary $(0)$ mode near the $m$-th harmonic, where $\lambda_{i}=k_{\perp} v_{\perp} / \omega_{c i}, J_{m}(\lambda)$ is the Bessel function of order $m, J_{m}^{\prime}(\lambda)=d J_{m}(\lambda) / d \lambda=\left[J_{m-1}(\lambda)-J_{m+1}(\lambda)\right] / 2, \theta$ is the angle between $k$ and $B$ (i.e., $\cos \theta=k_{1} / k$ ), $L^{3}$ is the plasma volume under study, and the "dressing" factor ${ }^{19,20}$ $\left.\mathbf{C}=\left[\mathbf{E}^{*} \cdot(1 / 2 \omega)(\partial / \partial \omega)\left(\omega^{2} \mathbf{K}_{\mathbf{h}}\right) \cdot \mathbf{E}\right) /\left(\mathbf{E}^{*} \cdot \mathbf{E}\right)\right]$. Here, $\mathbf{K}_{\mathbf{h}}$ is the Hermitian portion of the plasma dielectric tensor $\mathbf{K}, \mathbf{E}$ is wave electric field, and $\mathbf{E}^{*}$ is the complex conjugate of $\mathbf{E}$. This factor $\mathbf{C}$ takes account of the screening in the usual "dressed test particle" approach. 21 For $k_{\|} \ll k_{\perp}$ (i.e., $\theta \approx \pi / 2$ ), the index of refraction of the background medium 
$\mu=(\mathrm{ck} / \omega)=\mathrm{C}^{1 / 2}=\left[\left(\mathrm{E}^{*} \cdot \mathrm{K}_{\mathrm{h}} \cdot \mathrm{E}\right) /\left(\mathrm{E}^{*} \cdot \mathrm{E}\right)\right]^{1 / 2} \approx\left[\operatorname{Re}\left(\mathrm{K}_{\mathrm{yy}}+\mathrm{K}_{\mathrm{xy}}^{2} / \mathrm{K}_{\mathrm{xx}}\right)\right]^{1 / 2}$ for the $\mathrm{X}$-mode, and $\mu=(\mathrm{ck} / \omega)=\left[\left(\mathbf{E}^{*} \cdot \mathbf{K}_{\mathrm{h}} \cdot \mathbf{E}\right) /\left(\mathbf{E}^{*} \cdot \mathbf{E}\right)\right]^{1 / 2} \approx\left(\mathrm{R}_{\mathrm{e}} \mathrm{K}_{\mathrm{zz}}\right)^{1 / 2}$ for the O-mode, where Re stands for the real part and we have chosen $k$ to be in the $x-z$ plane. For $k_{\|} \gg k_{\perp}$ (i.e., $\theta \approx 0$ ), $\mu=(\mathrm{ck} / \omega)=\left[\left(\mathbf{E}^{*} \cdot \mathbf{K}_{\mathrm{h}} \cdot \mathbf{E}\right) /\left(\mathbf{E}^{*} \cdot \mathbf{E}\right)\right]^{1 / 2} \approx\left[\operatorname{Re}\left(\mathbf{K}_{\mathrm{xx}} \pm \mathrm{i} \mathbf{K}_{\mathrm{xy}}\right)\right]^{1 / 2}$, where the plus and minus signs correspond to the right and left circular polarizations, respectively. In the $O$ mode result of Eq. (4), $\mu^{-1} c=\omega / k=v_{p h}$ is the phase velocity of the emitted electromagnetic cyclotron harmonic wave in the background medium. In Eqs. (3) and (4) we have neglected the effects of magnetic curvature drifts. Since the $\delta$-functions of these equations are a consequence of the energy and momentum conservation, 18 it is relatively easy to show ${ }^{22}$ that taking account of the magnetic curvature drifts in these equations will result in the replacement of $\delta\left(\omega-m \omega_{c i}-k_{\|} v_{\|}\right)$by $\delta\left(\omega-m \omega_{c i}-k_{\|} v_{\|}-\omega_{\text {mcd }}\right)$ where $\omega_{\text {mcd }}=k \cdot v_{\text {mcd }}=k_{\perp} v_{m c d}$ is the magnetic curvature drift frequency corresponding to the magnetic curvature drift velocity $v_{\text {mcd. }}$. In a subsequent paper we will examine the magnetic curvature drift effects.

For $\lambda_{i}=k_{\perp} v_{\perp} / \omega_{c i}<1, J_{m}\left(\lambda_{i}\right) \approx(1 / m !)\left(\lambda_{i} / 2\right)^{m}$ and $J_{m}^{\prime}\left(\lambda_{i}\right) \approx(1 / m !)(m / 2)\left(\lambda_{j} / 2\right)^{m-1}$ $=\left(\mathrm{m} / \lambda_{\mathrm{i}}\right) \mathrm{J}_{\mathrm{m}}\left(\lambda_{\mathrm{i}}\right)$. Hence, $\left[\mathrm{J}_{\mathrm{m}+1}^{\prime}\left(\lambda_{\mathrm{i}}\right) / \mathrm{J}_{\mathrm{m}}^{\prime}\left(\lambda_{\mathrm{i}}\right)\right] \approx\left(\lambda_{\mathrm{i}} / 2 \mathrm{~m}\right)$ and $\left[\mathrm{v}_{\|} \mathrm{J}_{\mathrm{m}}\left(\lambda_{\mathrm{i}}\right) / \mathrm{v}_{\perp} \mathrm{J}_{\mathrm{m}}^{\prime}\left(\lambda_{\mathrm{i}}\right)\right] \Rightarrow$ $\left[\left(v_{\|} / v_{\perp}\right)\left(\lambda_{i} / m\right)\right]=\left(\lambda_{i} / m\right)$ for $v_{\perp}=v_{\|}$. Thus for $k_{\|}=0$, it is readily seen from Eqs. (3) and (4) that the ratio of the $O$ mode contribution to the intensity of each emission line near $\omega \approx m \omega_{c i}$ to that of the $\mathrm{X}$ mode $\approx\left(\lambda_{\mathrm{i}} / \mathrm{m}\right)^{2}=\left(\mathrm{v}_{\perp} / \mathrm{v}_{\mathrm{ph}}\right)^{2}$; while the ratio of the intensity of the $\mathrm{X}$ mode emission lines at $\omega \approx(m+1) \omega_{c i}$ to that at $\omega \approx m \omega_{c i}$ is approximately equal to $\left(\lambda_{i} / 2 m\right)^{2} \approx\left(v_{\perp} / 2 v_{p h}\right)^{2}$. That is, the ratio of the $O$ to $X$ mode contributions to the intensity of the $m$-th. harmonic $\approx(1 / 4)$ the ratio of the intensities of the $(m+1)$ th to the $m$-th harmonics. Further, it should be noted from Eq. (4) that the $O$ mode emission lines near $\omega=m \omega_{c i}$ will all show a fine structure, 18 being split into a doublet with a minimum around $\left(\mu^{-1} \mathrm{c} \cos \theta\right)=\left(v_{\mathrm{ph}} \cos \theta\right)=\left(\omega-\mathrm{m} \omega_{\mathrm{ci}}\right) / \mathrm{k}_{\|}$. Thus for $\left(\mathrm{v}_{\perp} / \mathrm{v}_{\mathrm{ph}}\right)^{2}$ $<1$, the $O$ mode contribution to each of the ICE lines at $\omega \approx m \omega_{c i}$ is small compared to that of the $X$ mode and hence the double-humped nature $18,2:$ of the "Trubnikov $O$ mode factor" will barely show up as a fine structure doublet splitting near the line centers. This expected picture is consistent with the JET observations mentioned earlier. However, since the fusion product ICE occurs in the background cooler thermal plasma medium, an alternative interpretation ${ }^{4}$ is the self absorption of the ICE line by the cooler background medium that have a relatively small Doppler width. We will see later that such self absorption is only possible at the fundamental frequency where the expected optical depth $\tau$ of the background medium is larger than unity, and for the higher harmonics such self absorption is impossible since $\tau \ll 1$. The diamagnetic drift frequency rotation of the plasma occurs only in one direction and these effects do not affect the $\delta$-functions of Eqs. (3) and (4). The diamagnetic effects alter the equilibrium distribution function via the guiding 
center perpendicular canonical momentum. 22 Consequently, the diamagnetic drift frequency cannot give rise to such doublet splitting of ICE lines as suggested in Ref. 4.

In Eqs. (3) and (4) we have used the conventional "box normalization" procedure. Hence the total spontaneous emission probability for the emission of a photon of frequency $\omega \approx$ ma $\omega_{c i}$ is given by

$$
\Sigma_{k} A(m) \rightarrow\left(\frac{L \mu}{2 \pi c}\right)^{3} \int d \Omega \int d \omega \omega^{2} A(m)
$$

where $d \Omega$ is the element of solid angle. Since $n \omega$ is the photon energy, the classical coefficient of spontaneous emission $\eta(m)$ of Eqs. (6.13) and (6.14) of Bekefi ${ }^{16}$ may be obtained from Eqs. (3-5) by using the relation

$$
\Sigma_{k}[\hbar \omega A(m)]=\int d \Omega \int d \omega \eta(m),
$$

where the transformation from Bekefi's mks system of units to our Gaussian system of units requires the replacement $\varepsilon_{0} \rightarrow(4 \pi)^{-1}$, and $d \Omega=2 \pi \sin \theta d \theta$ is the element of solid angle. Note that the Einstein $A$ and $B$ coefficients refer to the quantum mechanical transition probability for the emission of a photon while Bekefi's $\eta(m)$ coefficients represent the classical differential rate at which energy is emitted per unit solid angle per unit frequency interval, and this difference accounts for the relations of Eqs. (5) and (6). Hence, from Eqs. (5) and (6), Bekefi's classical coefficient of spontaneous emission for this test particle is

$$
\eta(m)=\left(\frac{L \mu \omega}{2 \pi c}\right)^{3} n A(m)
$$

If $n_{i}$ is the test particle number density and if $\left(2 \pi v_{\perp}\right) f\left(v_{\perp}, v_{\|}\right)=\left(2 \pi v_{\perp}\right) f_{\perp}\left(v_{\perp}\right) f_{\|}\left(v_{\|}\right)$is the normalized test particle velocity distribution function, then the total spontaneously radiated power per unit volume of these test particles in a particular harmonic $\mathrm{m}$ is given by

$$
P(m)=n_{i} \int d \Omega \int d \omega \int d v_{\perp}\left(2 \pi v_{\perp}\right) \int d v_{\|} \eta(m) f_{\perp}\left(v_{\perp}\right) f_{\|}\left(v_{\|}\right)
$$

Here we have made the reasonable assumption that the emitting test particles (i.e., the fusion ions) are nonrelativistic so that $f\left(v_{\perp}, v_{\|}\right)$is decomposable ${ }^{18}$ as the product $f_{\perp}\left(v_{\perp}\right) f_{\| 1}\left(v_{\|}\right)$. Such a 
decomposition is possible only in a homogeneous plasma model even for the nonrelativistic case. ${ }^{22}$ In a subsequent paper we will examine the effects of magnetic curvature and diamagnetic drifts where such a decomposition is not possible. In the plasma physics literature $16,24,25$ it is well known that in general it is extremely difficult if not impossible to carry out these cralculations analytically (i.e., the Bessel function averages over the velocity distribution functions) even with this decomposition of $f\left(v_{\perp}, v_{\|}\right)$for arbitrary angle $\theta$. Hence, for simplicity we will now examine the cases of $\theta=0, \theta \approx 0, \theta=\pi / 2$, and $\theta \approx \pi / 2$, respectively.

Case 1: $\theta=0$, i.e., $k_{\perp}=0$

Here since $k_{\perp}=0, \lambda_{i}=k_{\perp} v_{\perp} / \omega_{c i}=0, J_{m}\left(\lambda_{i}\right)=0$ for $m \geq 1, J_{1}^{\prime}\left(\lambda_{i}\right)=J_{0}(v) / 2=1 / 2$, and $J_{m}^{\prime}\left(\lambda_{i}\right)=0$ for $m \geq 2$. In the $O$ mode $A(m)$ of Eq. (4), the contribution from the component of the electric field $E=E_{z} i_{z} \propto\left(\mu^{-1} c \cos \theta-v_{\|}\right) J_{m}\left(\lambda_{i}\right)=0$. But, the contribution from the component of the electric field $E=E_{x} i_{x} \propto\left[\cos \theta\left(\mu-1 c \cos \theta-v_{\|}\right) / \sin \theta\right]$ $J_{m}\left(\lambda_{i}\right)=\left(\mu^{-1} c-v_{\|}\right)\left(k v_{\perp} / 2 \omega_{c i}\right)=\left(v_{\perp} / 2 \omega_{c i}\right)\left(\mu^{-1} c k-k v_{\|}\right)=\left(v_{\perp} / 2 \omega_{c i}\right)\left(\omega-k v_{\|}\right)=$ $\left(v_{\perp} / 2 \omega_{c i}\right) \omega_{c i}=v_{\perp} / 2$ for $m=1$, and is zero for $m \geq 2$, where we have used the $\delta$-function condition that $\omega-\omega_{c i}-k_{\|}=0$ for $m=1$ and $k=k_{\|}$since $\cos 0=1$. In Eq. (3), $v_{\perp} J_{m}^{\prime}\left(\lambda_{i}\right)=v_{\perp} / 2$ for $m=1$ and is zero for $m \geq 2$. Thus we see that for $\theta=0$, there is no emission for $m \geq 2$. There is ony a circularly polarized wave emission at the fundamental for $m=1$, and from Eqs. (3) and (4), we obtain

$$
\begin{aligned}
A(1) & =\left(\frac{4 \pi^{2} q_{i}^{2}}{L^{3} C \pi \omega}\right)\left|\frac{v_{\perp}\left(i_{x} \pm i i_{y}\right)}{2}\right|^{2} \delta\left(\omega-\omega_{c i}-k v_{\|}\right) \\
& =\left(\frac{4 \pi^{2} q_{i}^{2}}{L^{3} C h \omega}\right)\left|\frac{v_{1}^{2}}{2}\right| \delta\left(\omega-\omega_{c i}-k v_{\|}\right) .
\end{aligned}
$$

Thus, the Bekefi's classical coefficient of spontaneous emission for this test particle is $\eta(1)=(L \mu \omega / 2 \pi c)^{3} \hbar A(1)=\left(\mu^{3} / C\right)\left(q_{i}^{2} \omega^{2} / 4 \pi c^{3}\right) v_{\perp}^{2} \delta\left(\omega-\omega_{c i}-k v_{\|}\right) \approx\left(\mu q_{i}^{2} \omega^{2} / 4 \pi c^{3}\right) v_{\perp}^{2} \delta(\omega-$ $\left.\omega_{\mathrm{ci}}-k v_{\|}\right)$since $C \approx \mu^{2}$, and $\eta(m \geq 2)=0$. Since $v_{\perp}^{2}=(v \sin \theta)^{2}, \int d \Omega(\sin \theta)^{2}=8 \pi / 3$. Hence, from Eq. (8) we obtain the total spontaneusly radiated power per unit volume of these test particles in a given harmonic $m$ as 


$$
\begin{aligned}
P(1) & \approx\left(\frac{2 \mu n_{i} q_{i}^{2}}{3 c^{3}}\right) \int d \omega \omega^{2} \int d v_{\perp}\left(2 \pi v_{\perp}\right) f_{\perp}\left(v_{\perp}\right) v_{\perp}^{2} \int d v_{\|} f_{\|}\left(v_{\|}\right) \delta\left(\omega-\omega_{c i}-k v_{\|}\right) \\
& =\left(\frac{2 \mu n_{i} q_{1}^{2}<v_{\perp}^{2}>}{3 c^{3}}\right) \int d \omega \omega^{2}\left(\frac{1}{k}\right) f_{\| l}\left[v_{\|}=\frac{\left(\omega-\omega_{c i}\right)}{k}\right] \\
& =\left(\frac{2 n_{i} q_{i}^{2}<v_{\perp}^{2}>}{3 c^{2}}\right) \int d \omega \omega f_{\|}\left[v_{\|}=\frac{\left(\omega-\omega_{c i}\right)}{k}\right],
\end{aligned}
$$

and $P(m \geq 2)=0$. Here the angular brackets refer to a statistical average i.e., $\left\langle v_{\perp}^{2}\right\rangle=\int d v_{\perp}\left(2 \pi v_{\perp}\right) f_{\perp}\left(v_{\perp}\right) v_{\perp}^{2}=\left(2 \kappa T_{\perp i} / M_{i}\right)$, where $T_{\perp i}$ is the perpendicular temperature of these test particles and $k$ is the Boltzmann constant. In deriving Eq. (10) from Eq. (9) we have to integrate over all solid angles $\Omega$ to obtain the total radiated power. Since this is a dipole emission we have taken a standard dipole emission distribution in $d \Omega$, i.e., we have set $\int \mathrm{d} \Omega[]=2 \pi \int_{0}^{\pi} \sin$ $\theta d \theta(\sin \theta)^{2}\{\}=(8 \pi / 3)\{\}$, where we have written [ ] $=(\sin \theta)^{2}\{\}$. For cyclotron motion of the test ions with a Larmor radius $\rho_{L}$, the acceleration $a=v_{\perp}^{2} / \rho_{L}=\omega_{c i} v_{\perp}$, and hence the classical spontaneously radiated power $16,17.26$ by this test particle is $\left(2 q_{i}^{2} a^{2} / 3 c^{3}\right)=\left(2 q_{i}^{2} \omega_{c i}^{2} v_{\perp}^{2} / 3 c^{3}\right)$. This the result of Eq. (10) simply reflects the classical Doppler broadened spontaneous emission from a system of such test particles in a background medium of index of refraction $\mu$. This at least confirms the correctness of our Eqs. (3-8).

Case 2: $\theta \approx 0$, i.e., $k_{\perp} \ll k_{\|}$

Here since $k_{\perp} \ll k_{\|}, \lambda_{i}=k_{\perp} v_{\perp} / \omega_{c i} \ll 1, J_{m}\left(\lambda_{i}\right) \approx(1 / m !)\left(\lambda_{i} / 2\right)^{m}=(1 / m !)$ $\left(k_{\perp} / 2 \omega_{c i}\right)^{m} v_{\perp}^{m}$, and $j_{m}\left(\lambda_{i}\right) \approx(1 / m !)(m / 2)\left(\lambda_{j} / 2\right)^{m-1}=(1 / m !)(m / 2)\left(k_{\perp} / 2 \omega_{c i}\right)^{m-1} v_{\perp}^{m-1}$. We are particularly interested in the resonance of the fast Alfven wave of the background deuterium plasma with the $m$-th harmonic of the energetic fusion ion products (such as the protons and alpha particles), and hence the background index of refraction may be written

$$
\mu=\frac{c k}{\omega}=K_{h}^{1 / 2} \approx\left[1+\left(\frac{4 \pi n_{d} M_{d} c^{2}}{B^{2}}\right)\right]^{1 / 2}=\frac{c}{V_{A}},
$$

where the fast Alfven wave phase velocity $V_{A}=\omega / k=c /\left(1+4 \pi n_{d} M_{d} c^{2} / B^{2}\right)^{1 / 2} \approx$ $B /\left(4 \pi n_{d} M_{d}\right)^{1 / 2}, M_{d}$ is the mass of a deuteron, and $n_{d}$ is the number density of the background deuterium plasma ions. That is, we are inter sted in $\omega \approx m \omega_{\text {ci }} \leq \omega_{L H}$ where $\omega_{L H}$ is the lower 
hybrid frequency of the background plasma. In this case the allowed electromagnetic waves in the background medium are the fast hydromagnetic Alfven waves with the dispersion relation $\omega \approx k V_{A}$. Hence, for the $m$-th harmonic resonance of the test ions $\omega \approx m \omega_{c i},\left(k_{\perp} / 2 \omega_{c i}\right) \approx$ $(m k \sin \theta / 2 \omega)=\left(m \sin \theta / 2 V_{A}\right), J_{m}\left(\lambda_{i}\right) \approx(1 / m !)\left(m v_{\perp} \sin \theta / 2 V_{A}\right)^{m}$, and $J_{m}^{\prime}\left(\lambda_{i}\right) \approx(1 / m !)(m / 2)$ $\left(m v_{\perp} \sin \theta / 2 V_{A}\right)^{m-1}$. Thus on making use of Eqs. (3) and (4) in Eq. (8), we obtain the spontaneously radiated power per unit volume of these test particles in a given harmonic $m$ as

$$
\begin{aligned}
& P(m) \approx\left(\frac{4 \mu n_{i} q_{1}^{2}}{3 c^{3}}\right) \int d \omega \omega^{2} \int d v_{\perp}\left(2 \pi v_{\perp}\right) f_{\perp}\left(v_{\perp}\right)\left[\left(\frac{1}{m !}\right)^{2}\left(\frac{V_{A}}{\sin \theta}\right)^{2}\left(\frac{m v_{\perp} \sin \theta}{2 v_{A}}\right)^{2 m}\right] \\
& \int d v_{n} f_{\| 1}\left(v_{n}\right)\left[1+\left(\cos \theta-\frac{v_{H}}{v_{A}}\right)^{2}\right] \delta\left(\omega-m \omega_{c i}-k_{\|} v_{H}\right) \\
& =\left(\frac{4 n_{i} q_{i}^{2}}{3 c^{2} \cos \theta}\right)\left[\frac{m^{2 m}(\sin \theta)^{2 m-2} v_{A}^{2}}{(m !)^{2} 2^{2 m}}\right]\left[\left\langle\left(\frac{v_{1}}{V_{A}}\right)^{2 m}\right\rangle\right] \int d \omega \omega \\
& \left\{1+\left[\cos \theta-\frac{\left(\omega-m \omega_{c i}\right)}{V_{A} k \cos \theta}\right]^{2}\right\} f_{\| l}\left[v_{\| 1}=\frac{(\omega-1)}{k \cos \theta} \frac{\left.\omega_{c i}\right)}{k} .\right.
\end{aligned}
$$

For $\theta=0$, the factor $\left\{1+\left[\cos \theta-\left(\omega-m \omega_{c i}\right) / V_{A} k \cos \theta\right]^{2}\right\} \approx 2$ since $\omega \approx m \omega_{c i}$. Thus, it is relatively easy to show that Eq. (12) reduces to Eq. (10) for the case of $\theta=0$. For that case, we found that there is only emission at the fundamental cyclotron frequency corresponding to $\mathrm{m}=1$, and there is no emission at the harmonics corresponding to $m \geq 2$. But for $\theta \approx 0$ corresponding to a finite value of $k_{\perp} \ll k_{\|}$, there is emission at all the higher harmonics and the emission at the $\mathrm{m}$-th harmonic is proportional to $\left(\left\langle\mathrm{v}_{\perp} / \mathrm{V}_{\mathrm{A}}\right\rangle\right)^{2 \mathrm{~m}}=\left(\left\langle\mathrm{v}_{\perp}\right\rangle / \mathrm{V}_{\mathrm{A}}\right)^{2 \mathrm{~m}}$.

Case 3: $\theta=\pi / 2$, i.e., $k_{\|}=0$

Here since $k_{\|}=0, \mu=c k / \omega \approx c / V_{A}$, and by the $\delta$ functions of Eqs. (3) and (4) $\omega=m \omega_{c i}, \lambda_{i}=k_{\perp} v_{\perp} / \omega_{c i}=m k v_{\perp} / \omega \approx m v_{\perp} / v_{A}$. Thus on making use of Eqs. (3) and (4) in Eq. (8), we obtain the spontaneously radiated power per unit volume of these test particles in a given harmonic $m$ as 


$$
\begin{aligned}
P(m) \approx & \left(\frac{4 \mu n_{i} q_{i}^{2}}{3 c^{3}}\right) \int d \omega \omega^{2} \int d v_{\perp}\left(2 \pi v_{\perp}\right) f_{\perp}\left(v_{\perp}\right) \int d v_{\|} f_{\| l}\left(v_{\| l}\right) \\
& \left.\left\{\left[v_{\perp} J_{m}^{\prime}\left(\frac{m v_{\perp}}{v_{A}}\right)\right]^{2}+\left[v_{\| l} J_{m}\left(\frac{m v_{\perp}}{v_{A}}\right)\right]^{2}\right\} \delta\left(\omega-m \omega_{c i}\right) \approx\left(\frac{4 n_{i} q_{i}^{2} \omega_{c i}^{2} v_{A}}{3 c^{2}}\right)<G_{m}\right\rangle,
\end{aligned}
$$

where

$$
G_{m}\left[\left(\frac{v_{\perp}}{v_{A}}\right),\left(\frac{v_{\|}}{v_{A}}\right)\right]=\left\{\left[\left(\frac{m v_{\perp}}{v_{A}}\right) J_{m}^{\prime}\left(\frac{m v_{\perp}}{v_{A}}\right)\right]^{2}+\left[\left(\frac{m v_{\|}}{v_{A}}\right) J_{m}\left(\frac{m v_{\perp}}{v_{A}}\right)\right]^{2}\right\}
$$

$\mu \approx c / N_{A}$, and the angular brackets refer to the statistical average over the perpendicular and the parallel velocity distribution functions. In the $G_{m}$ of Eq. (14) the first term that is proportional to $\mathrm{J}_{\mathrm{m}}^{\prime}$ is the contribution of the extraordinary mode and the second term that is proportional to $v_{1} \mathrm{~J}_{\mathrm{m}}$ is the contribution of the ordinary mode. Since $x J_{m}^{\prime}(x)=m J_{m}(x)-x J_{m+1}(x), G_{m}$ of Eq. (14) can be rewritten as

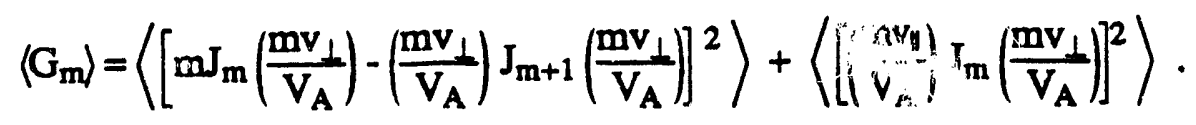

Chu and Sperling 27 have analyzed this problera using the monoenergetic velocity space ring distribution of the form $\left(2 \pi v_{\perp}\right) f_{\perp}\left(v_{\perp}\right)=\delta\left(v_{\perp}-v_{\alpha}\right)$ and $f_{\|}\left(v_{\|}\right)=\delta\left(v_{\|}\right)$. For this distribution function, our Eq. (14) or (14a) becomes

$$
\left\langle G_{m}\right\rangle=\left[\left(\frac{m V_{\alpha}}{V_{A}}\right) J_{m+1}\left(\frac{m V_{\alpha}}{V_{A}}\right)-m J_{m}\left(\frac{m V_{\alpha}}{V_{A}}\right)\right]^{2}
$$

Our results of Eqs. (13) and (15), differ slightly from the corresponding Chu and Sperling27 results of their Eqs. (23) and (24), respectively. For example, their Eq. (23) yields a value for $P(m)$ which is $\left(2 \pi^{2}\right)(3 / 4)=\left(3 \pi^{2} / 2\right)$ times larger than that given by our Eq. (13). Their Eq. (24) contains an extra term $\left(\mathrm{m}^{2} \omega_{\mathrm{c}} / \omega_{\mathrm{cb}}\right) \mathrm{J}_{\mathrm{m}}\left(\mathrm{mV}_{\alpha} / \mathrm{V}_{\mathrm{A}}\right)$ inside the square irackets on the right hand side compared to the corresponding ones of our Eq. (15), where $\omega_{\mathrm{cb}}$ is the cyclotron frequency of ions of the background medium. We do not know the origin of this factor. For example, if the background medium is vacuum, then $\omega_{\mathrm{cb}}=0$ since there are no ions in vacuum. But this terma is then infinite. Our Eqs. (13) and (14), and hence Eqs. (14a) and (15), agree exactly with the Trubnikov formulas. ${ }^{16,17}$ Further, we have shown in Case 1 of this section that the total emission 
agrees with the well-known classical electrodynamic fnrmula for the emission from an accelerated charge particle, 26 i.e., $P=\left(2 q^{2} a^{2} / 3 c^{3}\right)$, where $q$ and a are the particle's charge and acceleration, respectively. This shows the correctness of the factor $4 / 3$ on the right side of our Eq. (13) compared to the corresponding factor of $2 \pi^{2}$ in Eq. (23) of Chu and Sperling. Hence, we believe our Eqs. (13-15) is fully correct:

Case 4: For $\theta=\pi / 2$, i.e., $k_{\|} \ll k_{\perp}$

Here since $k=k_{\perp}, \omega=m \omega_{c i}, \mu=c / V_{A}$ and $V_{A}=\omega / k=m \omega_{c i} / k$, we may write $\lambda_{i}=k_{\perp} v_{\perp} / \omega_{c i}=k \sin \theta v_{\perp} / \omega_{c i}=m v_{\perp} / v_{A}$. Also in Eq. (4) we can set $\sin \theta \approx \sin \pi / 2 \approx 1$. Then on making use of Eqs. (3) and (4) in Eq. (8), we obtain the spontaneously radiater power pes unit volume of these test particles in a given harmonic $m$ as

$$
\begin{aligned}
& P(m)=\left(\frac{4 \mu n_{i} q_{1}^{2}}{3 c^{3}}\right) \int d \omega \omega^{2} \int d v_{\perp}\left(2 \pi v_{\perp}\right) f_{\perp}\left(v_{\perp}\right) \int d v_{11} f_{11}\left(v_{11}\right) \\
& \left\{\left[v_{\perp} J_{m}^{\prime}\left(\frac{m v_{\perp}}{v_{A}}\right)\right]^{2}+\left[(\sin \theta)^{-1}\left(v_{A} \cos \theta-v_{k}\right) J_{m}\left(\frac{m v_{\perp}}{v_{A}}\right)\right]^{2} \mid \delta\left(\omega-m \omega_{c i}-k \cos \theta v_{n}\right)\right. \\
& -\left(\frac{4 n_{i} q_{i}^{2} \omega_{c i}^{2} V_{A}}{3 c^{2}}\right) \int d \omega<\widetilde{G}_{m}\left[v_{\| l}=\frac{\left(\omega-m \omega_{c i}\right)}{k \cos \theta}\right]> \\
& \left(\frac{\omega}{m \omega_{c_{i}}}\right)^{2}\left(\frac{1}{k \cos \theta}\right) f_{1}\left[v_{11}=\frac{\left(\omega-m \omega_{c_{i}}\right)}{k \cos \theta}\right]
\end{aligned}
$$

where

$$
\begin{aligned}
\tilde{G}_{m}\left[\left(\frac{v_{d}}{v_{A}}\right),\left(\frac{v_{1}}{V_{A}}\right), \theta\right] & =\left\{\left[\left.\left(\frac{m v_{A}}{v_{A}}\right) j_{m}^{\prime}\left(\frac{m v_{d}}{v_{A}}\right)\right|^{2}\right\}\right. \\
& +\left\{\left[m \cos \theta-m\left(\frac{v_{11}}{v_{A}}\right)\right](\sin \theta)^{-1} J_{m}\left(\frac{m v_{1}}{v_{A}}\right)\right\}^{2} .
\end{aligned}
$$

In the limit $\theta \rightarrow \pi / 2, \cos \theta \rightarrow 0$, and $\sin \propto \int d v_{\|} f_{\|}\left(v_{\|}\right)=1$, it is relatively easy to show that Eqs. (16) and (17) reduce to Eqs. (13) and (14), respectively, as they should. 


\section{B. Spin-Flip Emission}

We stated earlier that in TFTR when deuterium beams were injected into a deuterium background plasma the main sequence of ICE harmonic peaks, which occur at the multiples of the deuterium cyclotron frequency $\omega_{c d-E}$ evaluated at tis outer low-field side plasma edge, seems to ride on a broader background continuum component of the spectrum that begins around the fift: harmonic of $\omega_{\text {cd-E. }}$. This "background continuum" emission exists roughly over the frequenc" range for which the proton spin-flip resonance is within the plasma. Since the proton gyromagnetic ratio or the $\mathrm{g}$ factor is equal to 5.59 , the fifth harmonic of $\omega_{c d-E}$ is near the frequency at which the proton spin-flip resonance is at the outer low-field side plasma edge. Hence, we now wish to examine the spontaneous spin-flip resonance emission from the fusion product protons as a possible mechanism for the observed background continuum spectrum seen in Fig. 1.

We, therefore, consider a Fenni particle such as a proton of mass $\mathrm{M}_{\mathrm{p}}$, charge $\mathrm{qp}_{\mathrm{p}}$, and spin $\mathrm{s}=$ $(\mathrm{h} / 2) \sigma$ in a uniform magnetic field $B=B \mathbf{i}_{z}$. Here $\sigma$ is the familiar Pauli spin matrix. The proton spin-flip resonance frequency $\omega_{s p}$ is then given by

$$
\omega_{s p}=\left(\frac{g_{p} q_{p} B}{2 M_{p} c}\right)=\left(\frac{g_{p}}{2}\right) \omega_{c p}=g_{p} \omega_{c d}
$$

where $g_{p}=5.59$ is the proton $g$ factor, and $\omega_{c p}$ and $\omega_{c d}$ are the proton and the deuteron cyclotron froquencies, respectively. The energy-level spectrum of this proton may be written 28

$$
E\left(m_{s}, v\right)=m_{s} \hbar \omega_{s p}+\frac{M_{p} v^{2}}{2}
$$

corresponding to the eigenstates $1 m_{s}, v>$, where $v$ is the proton's translational velocity, and $m_{s}$ can have only the two values $m_{3}=+1 / 2$ (spin-up) and $m_{3}=-1 / 2$ (spin-down). The spin-flip emission and absorption occurs when the proton makes a transition from the state $\operatorname{lm}_{\mathbf{s}}, \mathrm{v}>$ to another state $1 \mathrm{~m}_{3}^{\prime}, v^{\prime}>$ such that

$$
m= \pm\left(m_{s}^{\prime}-m_{s}\right)= \pm 1
$$

Let $A_{s}(m)$ be the Einstein's (quantum mechanical) spontaneous emission probability coefficient for the emission of a spin-flip photon of frequency $\omega \approx m \omega_{s p}= \pm \omega_{s p}$, and wave vector $k . A_{s}(m)$ is given by 28 


$$
A_{s}(m)=\left(\frac{4 \pi^{2} c^{2} k^{2}}{L^{3} C h \omega}\right)\left(\frac{g_{p} q_{p} \hbar}{4 M_{p} c}\right)^{2}|1 \pm i|^{2} \delta\left(\omega-m \omega_{s p}-k \cdot v\right)
$$

where we have neglected the Compton recoil velocity corrections of $\pm \hbar k / 2 \mathrm{M}_{\mathrm{p}}$ to the proton velocity v. The results of Eqs. (5-7) are also applicable to this spin-flip case. Let $f\left(m_{s}, v\right)=f_{s}\left(m_{s}\right) f_{v}(v)$ of normalized probability per unit volume that the protons in this box

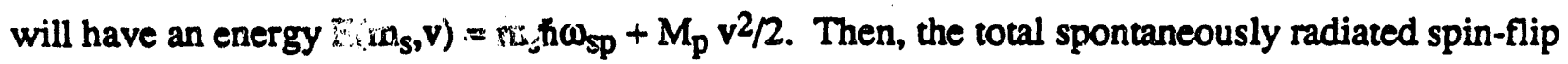
power per unit volume of these test fusion product protons in a given polarization $m= \pm 1$, corresponding to the spin-flip frequencies $\omega \approx \pm \omega_{\text {sp }}$, is given by

$$
P_{s}(m)=n_{p} f_{s}\left(m_{s}= \pm \frac{1}{2}\right) \int d \Omega \int d \omega \int d v f_{v}(v) \eta_{s}(m),
$$

where $\eta_{s}(m)$ is related to $A_{s}(m)$ by the same relation of Eq. (7), and $n_{p}$ is the proton number density. For a nondegenerate classical system at thermodynamic eanilibrium

$$
f_{s}\left(m_{s}\right)=\left[2 \cosh \left(\frac{\hbar \omega_{s p}}{2 K T_{s}}\right)\right]^{-1} \exp \left(\frac{-m_{s} \hbar \omega_{s p}}{K T_{s}}\right)
$$

and

$$
f_{v}(v)=\left(\frac{M_{p}}{2 \pi K T_{v}}\right)^{1 / 2} \exp \left[\frac{-M_{p}\left(v-V_{d}\right)^{2}}{2 K T_{v}}\right]
$$

where $T_{S}$ and $T_{V}$ are the temperatures appropriate to the spin and the translational degrees of freedom of the fusion protons, $V_{d}$ is their drift velocity, and $x$ is the Boltzmann constant. Thus, from Eqs. (7), (21), (22-24), we obtain the spontaneously radiated spin-flip power per unit volume of these test fusion product protons in the polarization $m=+1\left(i . e ., \omega \approx+\omega_{s p}\right)$ as

$$
\begin{aligned}
P_{s}(+1) & \left.\approx\left(\frac{4 \mu n_{p} q_{p}^{2}}{3 c^{3}}\right) f_{s}\left(+\frac{1}{2}\right)\left(\frac{g_{p}^{2}}{8}\right) \int d \omega \omega^{2}\left(\frac{\hbar k}{M_{p}}\right)^{2} \int d v f_{v}(v) \delta(\omega) \cdot \omega_{s p}-k \cdot v\right) \\
& =\left(\frac{4 \mu n_{p} q_{p}^{2}}{3 c^{3}}\right)\left(\frac{g_{p}^{2}}{8}\right) f_{s}\left(+\frac{1}{2}\right) \int d \omega \omega^{2}\left(\frac{\hbar k}{M_{p}}\right)^{2}|k|^{-1} f_{v}\left[|v|=\frac{\left(\omega-\omega_{s p}\right)}{k}\right] .
\end{aligned}
$$

It is physically instructive to compare the spin-flip result of Eq. (25) with the cyclotron result of Eqs. (10), (12) or (13). It is clear that the cyclorion emission is proportional to the square of the 
average perpendicular velocity of the particle $\left\langle v_{\perp}^{2}\right\rangle$, while the spin-flip emission is proportional to the square of the particle's Compton recoil velocity ( $\left.\pi \mathrm{k} / \mathrm{M}_{\mathrm{p}}\right)^{2}$. In order to make a rough consparison of the strength of spin-flip emission with that of the cyclotron emission, we will neglect the Doppler broadening effects in Eq. (25). Then, $\delta\left(\omega-\omega_{\text {sp }}-\mathbf{k} \cdot \mathbf{v}\right)$ tends to $\delta\left(\omega-\omega_{\text {sp }}\right)$ in Eq. (25). Since $\mu=c k / \omega \approx c / V_{A}$, we obtain the approximate spin-flip power as

$$
\begin{aligned}
P_{s}(+1) & \approx\left(\frac{4 n_{p} q_{p}^{2} \omega_{s p}^{2} V_{A}}{3 c^{2}}\right)\left(\frac{g_{p}^{2}}{8}\right) f_{s}\left(+\frac{1}{2}\right)\left(\frac{\hbar \omega_{s p}}{M_{p} V_{A}^{2}}\right)^{2} \\
& \approx\left(\frac{4 n_{p} q_{p}^{2} \omega_{c p}^{2} V_{A}}{3 c^{2}}\right)\left[\left(\frac{1}{2}\right)\left(\frac{g_{p}}{2}\right)^{6}\left(\frac{\hbar \omega_{c p}}{M_{p} V_{A}^{2}}\right)^{2} f_{s}\left(+\frac{1}{2}\right)\right] .
\end{aligned}
$$

Thus, from Eqs. (13) and (26), we find that

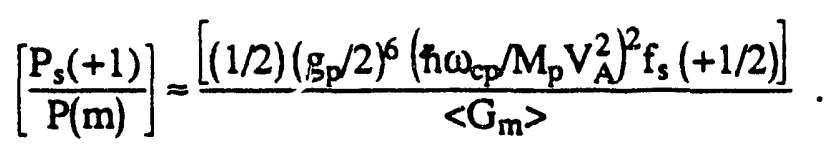

Since $\left\langle G_{1}\right\rangle \approx 2\left\langle\left(v_{\perp} / V_{A}\right)^{2}\right\rangle$, we find that $\left[P_{s}(+1) / P(1)\right] \approx 4.37 \times 10^{-24}$ for the typical tokamak parameters of Sec. VII if we assume that $f_{s}(+1 / 2) \approx 1 / 2$. The correctness of the result of Eq. (27) can be understood from first principles in the following way: The quantum mechanical photonproton interaction Hamiltonian that is responsible for transitions in which only one cyclotron light quanturs. is involved is $\mathrm{H}_{c \text {-int }}=-\left(\mathrm{q}_{\mathrm{p}} \mathbf{v} \cdot \mathbf{A} / \mathrm{c}\right)$, while the corresponding one for which only one spin-flip light quantum is involved is $H_{s-i n t}=\left(g_{p} q_{p} s \cdot \nabla \times A / 2 M_{p} c\right)=\left(g_{p} q_{p} h \sigma \cdot \nabla \times A / 4 M_{p} c\right)=$ $\left(g_{p} q_{p} \hbar \sigma \cdot k \times A / 4 M_{p} c\right)$. That is, $\left(H_{s-i n} / H_{c-i n t}\right)=\left(g_{p} \hbar k / 4 M_{p} v\right)$. The Einstein $A$ coefficient is proportional to $\mid \mathrm{H}_{\text {int }} t^{2}$, the $\Sigma_{k} \mathrm{~A}$ is proportional to $\int \mathrm{d} \omega \omega^{2} \mathrm{~A}$ which in turn is proportional to $\omega^{3} \mathrm{~A}$, and the emitted power $P$ is proportional to $\Sigma_{k} \hbar \omega A$. Also from Eq. (18), $\omega_{s p}=\left(g_{p} / 2\right) \omega_{c p}$. Hence, the ratio of the of the spin-flip emission to the cyclotron emission $\left(P_{s} / P_{c}\right)$ is proportional to $\omega^{4}\left|\mathrm{H}_{\mathrm{int}}\right|^{2}$ which in turn is proportional to $\left[\left(\mathrm{g}_{\mathrm{p}} / 2\right)^{4}\left(\mathrm{~g}_{\mathrm{p}} \hbar \mathrm{k} / 4 \mathrm{M}_{\mathrm{p}} \mathrm{v}_{\perp}\right)^{2}\right]$ $=\left\{\left[\left(\mathrm{g}_{\mathrm{p}} / 2\right)^{6}\left(\hbar \omega_{\mathrm{cp}} / \mathrm{M}_{\mathrm{p}} \mathrm{V}_{\mathrm{A}}^{2}\right)^{2}\right] / 4<\left(\mathrm{v}_{\perp} / \mathrm{V}_{\mathrm{A}}\right)^{2}>\right\} \approx\left\{\left[\left(\mathrm{g}_{\mathrm{p}} / 2\right)^{6}\left(\hbar \omega_{\mathrm{cp}} / \mathrm{M}_{\mathrm{p}} \mathrm{V}_{\mathrm{A}}^{2}\right)\right] / 2<\mathrm{G}_{\mathrm{m}}>\right\}$, since $\omega$ $=\omega_{\mathrm{cp}} \approx \mathrm{k} \mathrm{V}_{\mathrm{A}}$. Thus, we see the correctness of the result of Eq. (27) follows trivially from the fundamental physical principles. Earlier we have shown that our result of Eq. (10) agrees exactly with the well-known classical emission formula from an accelerated charged particle $\left(2 q^{2} a^{2} / 3 c^{3}\right)$, as found in standard text books on classical electromagnetic theory. ${ }^{26}$ 


\section{Evaluation of Statistical Averages for Various Distributions}

In this section we wish to carry out the explicit evaluation of the statistical averages implied by the angular brackets $\langle\cdots\rangle$ in the earlier sections. These angular brackets refer to statistical averages over the appropriate perpendicular and parallel velocity space distribution functions. First, we consider the monoenergetic velocity space ring (or, equivalently, a cylindrical shell) distribution of Chu and Sperling. 27 Second, we consider the monoenergetic isotropic velocity space spherical shell distribution of Dendy, Lashmore-Davies, and Kam, ${ }^{29}$ and third, we consider the conventional anisotropic $\left(T_{\perp} \neq T_{11}\right)$ Maxwell-Boltzmann distribution function. These monoenergetic distribution functions are a good approximation to the newly born fusion products such as protons and alpha particles, while the anisotropic Maxwelliar. disiribution can well describe the spontaneous emission from the background deuterium plasma ions. We should point out that, after some period of time from their birth, these fusion products will relax towards the slowingdown distribution function 30 of the form $f(v)=\left(A_{d} / v_{c}\right)\left[1+\left(v / v_{c}\right)\right]^{-3}$ for $v<v_{m}$ and $f(v)=0$ for $v>v_{m}$, where $v_{m}=\left(2 E_{\alpha} / M_{\alpha}\right)^{1 / 2}$ is the maximum velocity corresponding to the birth energy $E_{\alpha}$ of the fusion product of mass $M_{\alpha}, v_{c}=\left(3 \pi^{1 / 2} Z_{\text {eff }} M_{e} / M_{\alpha}\right)^{1 / 3} v_{e}$, and $A_{0}=3 /\left\{4 \pi \ell n\left[1+\left(v_{c} / v_{m}\right)^{-3}\right]\right\}$ Here, $v_{e}=\left(2 x T_{e} / M_{e}\right)^{1 / 2}$ is the electron thermal speed, and $Z_{\text {eff }}=\Sigma_{i}\left(n_{i} Z_{i}^{2} / n_{e} M_{i}\right)$ is the effective value of the ionic charge. For the sake of analytical simplicity, we will only consider the monnenergetic ring, monoenergetic spherical shell, and Maxwellian distributions here. The spontaneous emiss on depends only on the average moments of the distribution function [such as, for exam $m_{k i}, 2 m$-thmoment $\left\langle\left(v_{\perp}^{2 m}\right)>\right.$ ] and is hence very insensitive to the slopes and shapes of the distribution function, while the balance between the induced emission and absorption which determines the growth or damping of the cyclotron harmonic waves (to be discussed in a later section) can be a sensitive function of the slope and shape of the distribution function. For these reasons, the introduction of a small thermal spread to the above two monoenergetic cases considered here will not appreciably alter our conclusions about the spontaneously emitted power.

\section{Case 1: Monoenergetic Ring Distribution}

Chu and Sperling 27 have used a monoenergetic ring or, equivalently, a cylindrical shell distribution in perpendicular velocity space and this may be written

$$
f(v)=\left(2 \pi v_{\perp}\right)^{-1} \delta\left(v_{\perp}-v_{i}\right) \delta\left(v_{\|}\right)
$$


Here, of course, the statistical averaging is trivial and $\left\langle v_{\perp}^{2}\right\rangle=V_{i}^{2}$ in Eq. (10); $\left\langle v_{\perp}^{2 m}\right\rangle=V_{i}^{2 m}$ in Eq. (12); and $\left.\left\langle v_{i}^{2}\right\rangle=0,<\left[J_{m}\left(m v_{\perp} / V_{A}\right)\right]^{2}\right\rangle=\left[J_{m}\left(m V_{i} / V_{A}\right)\right]^{2}$, $<\left[\left(m v_{\perp} / V_{A}\right) J_{m}^{\prime}\left(m v_{\perp} / V_{A}\right)\right]^{2}>=\left[\left(m V_{i} / V_{A}\right) J_{m}^{\prime}\left(m V_{i} / V_{A}\right)\right]^{2}$ in Eq. (14).

\section{Case 2: Monoenergetic Isotropic Spherical Shell Distribution}

Dendy, Lashmore-Davies, and $\mathrm{Kam}^{29}$ have used a monoenergetic isotropic velocity space spherical shell distribution as given by $f(v)=\left(4 \pi v^{2}\right)^{-1} \delta\left(v-V_{i}\right)$. However, in our treatment we have thus far retained the $(\perp, I)$ anisotropy and hence we will adopt this spherical shell distribution as given by

$$
f(v)=\left(2 \pi v_{\perp}\right)^{-1} \delta\left(v_{\perp}-v_{i}\right) \delta\left(v_{\|}-2^{-1 / 2} v_{i}\right)
$$

Note that according to Eq. (29), $v_{x}=v_{y}=v_{z}=2-1 / 2 v_{i}$. Hence Eq. (29) represents a monoenergetic isotropic velocity space spherical shell distribution with a mean velocity $v=\left(v_{x}^{2}+v_{y}^{2}+v_{z}^{2}\right)^{1 / 2}=(3 / 2)^{1 / 2} v_{i}$. That is, Eq. (29) is equivalent to the distribution $f(v)=\left(4 \pi v^{2}\right)^{-1} \delta\left[v-(3 / 2)^{1 / 2} v_{i}\right]$. In this case, all the perpendicular velocity averages are the same as the previous case 1 , and in Eq. (14) $\left\langle v_{\|}^{2}\right\rangle$ becomes $V_{i}^{2} / 2$. That is, $\left\langle G_{m}\right\rangle=\left\{\left[\left(m V_{j} / V_{A}\right) J_{m}^{\prime}\left(m V_{j} / V_{A}\right)\right]^{2}+\left[\left(m V_{j} / V_{A}\right) J_{m}\left(m V_{i} / V_{A}\right)\right]^{2} / 2\right\}$. The behavior of $\left\langle G_{m}>\right.$ as $a$ function of the harmonic number $m$ for $\left(V_{i} / V_{A}\right)$ values of $0.5,1.0,1.5,2.0,2.5$, and 3.0 are shown in Figs. 2(a), 2(b), 2(c), 2(d), 2(e) and 2(f), respectively. Fo. the typical tokamak parameters used in Sec. VII, the value of $\left(V_{j} / V_{A}\right)=2.28$. These plots are similar to those given by Chu and Sperling 27 but for a finite $\left\langle v_{\|}^{2}\right\rangle=2-1\left\langle v_{\perp}^{2}\right\rangle=v_{i}^{2} / 2$ instead of $\left\langle v_{\|}\right\rangle=0$ as assumed by $\mathrm{Chu}$ and Sperling. As we stated earlier, even if we introduce a thermal spread of $\varepsilon=\left[<(\Delta v)^{2}>\right] \ll v_{i}^{2}$ to the $\delta$ function distributions of Eqs. (28) and (29), the above results of cases 1 and 2 for the spontaneously emitted power by the fusion products will not be appreciably altered. That is, even if we replace $\delta\left(v-V_{i}\right)$ by the function $f(v)=\left\{(\pi \varepsilon)^{-1 / 2} \exp \left[-\left(v-V_{i}\right)^{2} / \varepsilon\right]\right\}$ where $\varepsilon \ll V_{i}$, the above results will not change significantly. Of course, the spectrum will no longer consist of sharp $\delta$-function lines at each harmonic but will be Doppler broadened with a full width at half maximum of $(\Delta \omega / \omega)=\left(2 k_{\|} \varepsilon^{1 / 2} / \mathrm{m} \omega_{c i}\right)$. However, the "strength" of every harmonic resonance $m$ is unchanged. By "strength" of the resonance we mean the frequency integral under the resonance curve. The reason for this is that in the limit $\varepsilon \rightarrow 0$, $f(v)=\left\{(\pi \varepsilon)^{-1 / 2} \exp \left[-\left(v-V_{i}\right)^{2} / \varepsilon\right]\right\} \rightarrow \delta\left(v-V_{i}\right)$. Thus, the Doppler shift of $\delta\left(\omega-m \omega_{c i}-k_{\|} V_{\|}\right)$ does a one to one mapping of $v_{\|}$to $v_{\|}=\left(\omega-m \omega_{c i}\right) / k_{\|}$, preserving the normalization condition integrals and the resonance strength. Note that the thermal spreading of the perpendicular $\delta$-function velocity distribution gives no change at all and it is only the spreading of the parallel $\delta$-function velocity distribution that will cause the Doppler broadening. 


\section{Case 3: Anisotropic Maxwell-Boltzmann Distribution}

As we stated earlier this distribution can well describe the background deuterium plasma ions and may be written $f\left(v_{\perp}, v_{\|}\right)=f_{\perp}\left(v_{\perp}\right) f_{\|}\left(v_{\|}\right)$, where

$$
f_{\perp}\left(v_{\perp}\right)=\left(\frac{M_{i}}{2 \pi \kappa T_{\perp i}}\right) \exp \left[\frac{-M_{i} v_{\perp}^{2}}{2 \kappa T_{\perp i}}\right] ; 0 \leq v_{\perp} \leq \infty
$$

and

$$
f_{\|}\left(v_{\|}\right)=\left(\frac{M_{i}}{2 \pi \kappa T_{\| i}}\right)^{1 / 2} \exp \left[\frac{-M_{i}\left(v_{\|}-V_{z}\right)^{2}}{2 \kappa T_{\| i}}\right] ;-\infty \leq v_{\|} \leq \infty .
$$

Here, $V_{z}$ is a drift velocity in the $z$ direction. In performing the integration over $v_{\perp}$, we will make use of the following formula given by Watson 31

$$
\int_{0}^{\infty} \rho d \rho J_{n}\left(s_{1} \rho\right) J_{n}\left(s_{2} \rho\right) \exp \left(-\rho^{2}\right)=\left(\frac{1}{2}\right) \exp \left[-\frac{s_{1}^{2}+s_{2}^{2}}{4}\right] I_{n}\left(\frac{s_{1} s_{2}}{2}\right),
$$

where $I_{n}(x)=i^{-n} J_{n}(i x)$ is the modified Bessel function of the first kind. From this formula may be derived the three integrals necessary for the $v_{\perp}$ integration. These are:

$$
\begin{aligned}
& \int_{0}^{\infty} \rho d \rho\left[J_{n}(s \rho)\right]^{2} \exp \left(-\rho^{2}\right)=\left(\frac{1}{2}\right) \exp \left(\frac{-s^{2}}{2}\right) I_{n}\left(\frac{s^{2}}{2}\right) \\
& \int_{0}^{\infty} \rho^{2} d \rho\left[2 J_{n}(s \rho) J_{n}(s \rho)\right] \exp \left(-\rho^{2}\right)=\left(\frac{s}{2}\right) \exp \left(\frac{-s^{2}}{2}\right)\left[I_{n}^{\prime}-I_{n}\right]
\end{aligned}
$$

and

$$
\int_{0}^{\infty} \rho^{3} d \rho\left[J_{n}^{\prime}(s \rho)\right]^{2} \exp \left(-\rho^{2}\right)=\left(\frac{s^{2}}{8}\right) \exp \left(\frac{-s^{2}}{2}\right)\left[\left(2+\frac{4 n^{2}}{s^{4}}\right) I_{n}-I_{n}^{\prime}\right]
$$

In Eqs. (34) and (35), the prime denotes a derivative with respect to the argument and the argument of $I_{n}$ and $I_{n}^{\prime}$ is $\left(s^{2} / 2\right)$. Note that in Eq. $\left.(34),\left[2 J_{n}(s \rho) J_{n}^{\prime}(s \rho)\right]=\left[d J_{n}^{2}(s \rho)\right] / d(s \rho)\right]$. 
Hence, in Eq. (10), $\left\langle v_{\perp}^{2}\right\rangle=\left(2 \kappa \mathrm{T}_{\perp \mathrm{i}} / \mathrm{M}_{\mathrm{i}}\right)$; in $\mathrm{Fq} .(12),\left\langle\mathrm{v}_{\perp}^{2 \mathrm{~m}}\right\rangle=\left(2 \kappa \mathrm{T}_{\perp \mathrm{i}} / \mathrm{M}_{\mathrm{i}}\right)^{\mathrm{m}} \mathrm{m}$ !; and in Eq. (14), $\left\langle v_{\|}^{2}\right\rangle=\left[\left\langle\left(v_{\|}-V_{z}\right)^{2}\right\rangle+2\left\langle v_{\|}\right\rangle V_{z}-V_{z}^{2}\right]=\left[\left(2 \kappa T_{\| i} / M_{i}\right)+V_{z}^{2}\right]$ since $\left\langle\mathrm{v}_{\|}\right\rangle=\mathrm{v}_{\mathrm{z}},\left\langle\left[\mathrm{J}_{\mathrm{m}}\left(\mathrm{mv_{ \perp }} / \mathrm{V}_{\mathrm{A}}\right)\right]^{2}\right\rangle=\left[\exp \left(-\mathrm{s}_{\mathrm{m}}^{2} / 2\right) \mathrm{I}_{\mathrm{m}}\left(\mathrm{s}_{\mathrm{m}}^{2} / 2\right)\right],\left\langle\left[\left(\mathrm{mv}_{\perp} / \mathrm{V}_{\mathrm{A}}\right) \mathrm{J}_{\mathrm{m}}^{\prime}\left(m v_{\perp} / \mathrm{v}_{\mathrm{A}}\right)\right]^{2}\right\rangle$ $=\left(s_{m}^{4} / 4\right) \exp \left(-s_{m}^{2} / 2\right)\left[\left(2+4 m^{2} / s_{m}^{4}\right) I_{m}\left(s_{m}^{2} / 2\right)-I_{m}^{\prime}\left(s_{m}^{2} / 2\right)\right]$ where $s_{m}=\left(m / V_{A}\right)\left(2 \kappa T_{\perp i} / M_{i}\right)^{1 / 2}$. Thus for $\theta=\pi / 2$ and $\theta \approx \pi / 2$, the spontaneously radiated power per unit volume of a Maxwellian distribution of particles in a given harmonic $m$ is given by Eqs. (13) and (16), where $<G_{m}>$ and $<\widetilde{G}_{m}>$ of Eqs. (14) and (17) become

$$
<G_{m}>\approx \exp \left(\frac{-s_{m}^{2}}{2}\right)\left\{m^{2} I_{m}+\left(\frac{s_{m}^{4}}{4}\right)\left(2 I_{m}-I_{m}^{\prime}\right)+\left[\frac{V_{z}^{2}+\left(2 \kappa T_{l i j} / M_{i}\right)}{V_{A}^{2}}\right] m^{2} I_{m}\right\},
$$

and

$<\widetilde{G}_{m}>\approx \exp \left(\frac{-s_{m}^{2}}{2}\right)\left\{m^{2} I_{m}+\left(\frac{s_{m}^{4}}{4}\right)\left(2 I_{m}-I_{m}^{\prime}\right)+\left[\cos \theta-\frac{\left(\omega-m \omega_{c i}\right)}{\omega \cos \theta}\right]^{2}(\sin \theta)^{-2} m^{2} I_{m}\right\}$,

respectively. Here again the argument of $I_{n}$ and $I_{n}^{\prime}$ is $\left(s_{m}^{2} / 2\right)$ where $s_{m}=\left(m_{\perp} / \omega\right)\left(2 \kappa T_{\perp i} / M_{i}\right)^{1 / 2}=$ $(m k \sin \theta / \omega)\left(2 \kappa T_{\perp i} / M_{i}\right)^{1 / 2} \approx\left(m / V_{A}\right)\left(2 \kappa T_{\perp i} / M_{i}\right)^{1 / 2}$ for near perpendicularly propagating fast Alfven waves, and the prime denotes a derivative with respect to the argument.

\section{LINEARIZED THEORY OF RADIATIVE STEADY STATE}

In this section we first examine the linearized theory of the radiative steady state and/or radiative equilibrium due to the cyclotron harmonic emission and absorption by both the background deuterium plasma ions and the fusion product protons and/or alpha particles. Second, we then examine the similar radiative steady state of spin-flip emission and absorption of photons by the fusion product protons. In both cases we will indicate the conditions for instability leading to the cyclotron harmonic laser or maser and the spin-flip laser or maser, respectively.

\section{A. For Cyclotron Emission and Absorption}

Thus far we have only examined the spontaneous emission of radiation from individual "dressed test particles." This spontaneous emission analysis applies equally well both for background deuterium plasma ions and for newly born fusion products such as protons and alpha particles. For the newly born fusion product ions, the above spontaneous emission calculations of the monoenergetic velocity space ring and/or the monoenergetic isotropic velocity space spherical shell distributions are appropriate, while for the background deuterium plasma ions those of the Maxwellian distribution calculations should apply. These spontaneous emissions depend only on 
the various moments of the distribution function and are very insensitive to the nature of the slopes and shapes of these distribution functions. However, the growth or damping of the cyclotron harmonic fast Alfven waves under consideration can depend sensitively on the slopes and shapes of the distribution functions since these are determined by the balance between induced or stimulated emission and absorption. Thus the collective cooperative emission from a statistical system is a consequence of a detailed balance between these three processes of spontaneous emission, induced or stimulated emission and absorption. Indeed at the radiative steady state and/or at radiative equilibrium, the total cooperative spontaneous plus stimulated emissions exactly balances the total cooperative absorption, and the system as a whole emits from the surface as a black-body which can be characterized by a radiation temperature $T_{r}(\omega, k)$. It is this problem of the radiative steady state and/or the radiative equilibrium which we wish to examine in this section.

An analogous problem of the radiation temperature for electron cyclotron emission from equilibrium and nonequilibrium plasmas has been recontly analyzed in the literature by one of the authors (V.A.). ${ }^{18}$ From the resuits found in Ref. 18, it is relatively easy io show that the rate of increase of photons $\left[\mathrm{dN}\left(\omega \approx \mathrm{m} \omega_{\mathrm{ci}}, \mathrm{k}\right) / \mathrm{dt}\right]_{\mathrm{sem}}$ in. the box of volume $\mathrm{L}^{3}$ under consideration due to the spontaneous emission of photons of wave vector $k$ near the m-th harmonic (i.e., $\omega \approx m \omega_{c i}$ ) may be written

$$
\left[\frac{d N\left(\omega \approx m \omega_{c i}, k\right)}{d t}\right]_{s e m}=\int d v_{\perp}\left(2 \pi v_{\perp}\right) \int d v_{\|}\left[L^{3} n_{i} A(m)\right] f_{\perp}\left(v_{\perp}\right) f_{\|}\left(v_{k}\right)=\left\langle\left[L^{3} n_{i} A(m)\right]\right\rangle
$$

and the rate of decrease of these protons $\left\{\left[\mathrm{dN}\left(\omega \approx m \omega_{\mathrm{ci}}, \mathrm{k}\right) / \mathrm{dt}\right]_{\mathrm{ab}}-\left[\mathrm{dN}\left(\omega \approx \mathrm{m} \omega_{\mathrm{ci}}, \mathrm{k}\right) / \mathrm{dt}\right]_{\mathrm{iem}}\right\}$ in this box due to absorption minus the induced emission of photons of wave vector $k$ near the $m$-th harmonic is given by

$$
\begin{aligned}
&\left.\int\left[\frac{d N\left(\omega \approx m \omega_{c i}, k\right)}{d t}\right]_{a b}-\left[\frac{d N\left(\omega \approx m \omega_{c i}, k\right.}{d t}\right]_{i e m}\right]=-\left\{\int d v_{\perp}\left(2 \pi v_{\perp}\right) \int d v_{\|}\left[L^{3} n_{i} A(m)\right]\right. \\
& {\left.\left[\frac{m \hbar \omega_{c i}}{M_{i} v_{\perp}}\left(\frac{\partial}{\partial v_{\perp}}\right)+\left(\frac{\hbar k_{\|}}{M_{i}}\right)\left(\frac{\partial}{\partial v_{\|}}\right)\right] f_{\perp}\left(v_{\perp}\right) f_{\| l}\left(v_{\| l}\right)\right\} N\left(\omega \approx m \omega_{c i}, k\right) } \\
&=<\left\{\left(\frac{m \hbar \omega_{c i}}{M_{i} v_{\perp}}\right)\left(\frac{\partial}{\partial v_{\perp}}\right)\left[L^{3} n_{i} A(m)\right]+\left(\frac{\hbar k_{\| l}}{M_{i}}\right)\left(\frac{\partial}{\partial v_{\|}}\right)\left[L^{3} n_{i} A(m)\right]\right\} N\left(\omega \approx m \omega_{c i}, k\right) \\
& \equiv 2 \gamma\left(\omega \approx m \omega_{c i}, k\right) N\left(\omega \approx m \omega_{c i}, k\right),
\end{aligned}
$$


where $\gamma\left(\omega \approx m \omega_{c i}, k\right)$ is the damping rate of the ion cyclotron harmonic fast Alfven waves whose optical depth $\tau$ may be writien as

$$
\tau\left(\omega \approx m \omega_{c i}, k\right)=\int d \ell\left(\frac{\gamma}{V_{A}}\right)
$$

since the group velocity of these fast Alfven waves is the same as their phase velocity $V_{A}$, and $d \ell$ is the incremental path length of the radiation 6 .ld in the medium under study. In arriving at the second equality of Eq. (39) we have carried out two integrations by parts, one over $d v_{\perp}$ and the other $\mathrm{dv} \|$. It should be noted from the A(m)'s of Eqs. (3) and (4) that the particle-wave resonance occurs only in the parallel velocity distribution function at the parallel cyclotron phase velocity $v_{p}=v_{\|}=\left(\omega-m \omega_{c i}\right) / k_{\|}$. Hence, contrary to the intuitive expectations 3,22 the damping rate $\gamma$ of Eq. (39) is not sensitive to the shapcs and slopes of the perpendicular velocity space distribution function. It is, however, weakly sensitive to the slope of the parallel velocity space distribution around $v_{\|}=v_{p} \approx\left(\omega-m \omega_{c i}\right) / k_{\|}$, and this usually manifests itself as the familiar cyclotron overstability terms $18,24,25$ in the conventional hot plasma theory. 24,25

At the radiative steady state, the rate of increase of photons due to the spontaneous emission of Eq. (38) is exactly equal to the rate of decrease of these photons due to absorption minus the induced emission of Eq. (39). That is, at the radiative steady state the net rate of increase of photons of frequency $\omega$ and wave vector $k$ given by 14

$$
\left[\frac{\mathrm{dN}(\omega, k)}{\mathrm{dt}}\right]=\left[\frac{\mathrm{dN}(\omega, k)}{\mathrm{dt}}\right]_{\mathrm{sem}}-\left\{\left[\frac{\mathrm{dN}(\omega, k)}{\mathrm{dt}}\right]_{\mathrm{ab}}-\left[\frac{\mathrm{dN}(\omega, k)}{\mathrm{dt}}\right]_{\mathrm{iem}}\right\}
$$

is equal to zero. Then the radiation temperature $T_{r}\left(\omega=m \omega_{c i}, k\right)$ for these fast Alfven waves may be defined as

$$
\begin{aligned}
& \left(\frac{\kappa T_{r}}{n \omega}\right)=\frac{\left[d N\left(\omega \approx m \omega_{c i}, k\right) / d t\right]_{s e m}}{\left[2 \gamma\left(\omega \approx m \omega_{c i}, k\right)\right]} \\
& =\frac{\left\langle\left[L^{3} n_{i} A(m)\right]\right\rangle}{\left\langle\left(m \hbar \omega_{c i} / M_{i} v_{\perp}\right)\left(\partial / \partial v_{\perp}\right)\left[L^{3} n_{i} A(m)\right]+\left(\hbar k_{\|} / M_{i}\right)\left(\partial / \partial v_{\|}\right)\left[L^{3} n_{i} A(m)\right]\right\rangle} .
\end{aligned}
$$


Here again we wish to emphasize that the Dirac's $\delta$ functions of Eqs. (3) and (4) yield the conventional cyclotron and/or Landau damping which depends on the slope of the parallel velocity distribution function at $v_{\|} \approx v_{p} \approx\left(\omega-m \omega_{c i}\right) / k_{\|}$, and this in turn yields the familiar cyclotron overstability terms. Since the relevant physical quantities depend only on the moments of $f_{\perp}\left(v_{\perp}\right)$ as in Eq. (42), for example, these quantities are very insensitive to the slope of the perpendicular velocity distribution function.

If the receiving antenna is only sensitive to a given $\mathrm{k}$ direction (i.e., is one dimensional) then the received radiated power in a frequency bandwidth $\Delta \omega / 2 \pi$ is $P(m)=(\Delta \omega / 2 \pi)\left(\kappa T_{r}\right)(1-\zeta)[1-\exp (-\tau)] /[1-\zeta \exp (-\tau)]$, where $\tau$ is the optical depth and $\zeta$ is the wall reflection coefficient. If the antenna is two dimensional, then the received radiated power in the frequency bandwidth $\Delta \omega$ centered around the frequency $\omega$ is $P(m) \propto(\omega \Delta \omega)$, and if the antenna is three dimensional then it is given by $P(m) \propto\left(\omega^{2} \Delta \omega\right)$. These differences in $\mathrm{P}(\mathrm{m})$ are of course due to the fact ${ }^{16}$ that in one dimension the phase space volume is $\mathrm{dk}$, in two dimensions it is $2 \pi \mathrm{kdk}$, and in three dimensions it is $4 \pi \mathrm{k}^{2} \mathrm{dk}$. This phase space volume is the measure of the number of plane waves per unit volume per unit frequency interval $d \omega$, and by the classical equipartition theorem the energy per wave is $k T$. However, since this cyclotron harmonic emission for $m \geq 2$ is purely due to the finite value of $k_{\perp}$ and most of this emission is in the $k_{\perp}$ direction, it would appear that the system under study would tend to behave as a two dimensional black-body system even if the antenna is three dimensionally sensitive.

It may be noted from Eqs. (38), (39), and (41) that if the absorption exceeds the induced emission, then $\gamma$ is positive (i.e., the cyclotron harmonic fast Alfven waves are damped) and the system will reach a radiative steady state with a finite value for the wave energy density $\varepsilon\left(\omega \approx m \omega_{c i}, k\right)=\hbar \omega N\left(\omega \approx m \omega_{c i}, k\right) / L^{3}$. In this linearized theory, when the absorption is exactly equal to the induced emission, the system is at marginal stability (i.e. $\gamma=0$ ) and it is relatively easy to see from Eqs. (38), (39), and (41) that the steady state wave energy density $\varepsilon(\omega, k)=\hbar \omega N(\omega, k) / L^{3}$ tends to infinity. If the distribution function $f\left(v_{\perp}, v_{\|}\right)$is such that the induced emission exceeds the absorption in Eq. (39), then the system is unstable and behaves as a cyclotron harmonic fast Alfven wave laser or maser. In a subsequent section we will present the appropriate coupled pair of quasilinear equations that will govern the time evolution of such a laser or maser system.

\section{B . For Spin-flip Emission and Absorption}

The analogous problem of the electrodynamic properties of a gas of spin $1 / 2$ particles in a uniform external magnetic field has again been analyzed previously by one of the authors 
(V.A.). ${ }^{28}$ The distribution function $f\left(m_{S}, v\right)=f_{S}\left(m_{S}\right) f_{v}(v)$, and let $F_{S}(m)=\left[f_{S}\left(m_{S}\right)-f_{S}\left(-m_{S}\right)\right]$ be the fractional excess of particles between the spin-up and the spin-down states. Then it is relatively easy to show from Eq. (74) of Ref. 28 that the rate of increase of spin-flip excitations due to spontaneous emission is given by

$$
\left[\frac{d N\left(\omega \approx m \omega_{s p}, k\right)}{d t}\right]_{s e m}=\int d v\left[L^{3} n_{p} A_{s}(m)\right] f_{s}\left(m_{s}\right) f_{v}(v)
$$

and the rate of decrease due to the absorption minus the induced emission is

$$
\begin{aligned}
& \left.\int\left[\frac{d N\left(\omega \approx m \omega_{s p}, k\right)}{d t}\right]_{a b}-\left[\frac{d N\left(\omega \approx m \omega_{s p}, k\right)}{d t}\right]_{i e m}\right]=-\int d v\left[L^{3} n_{p} A_{s}(m)\right] \\
& {\left[f_{s}\left(m_{s}\right)-f_{s}\left(-m_{s}\right)\right] f_{v}(v) N\left(\omega=m \omega_{s p}, k\right)=-\int d v\left[L^{3} n_{p} A_{s}(m)\right]} \\
& F_{s}(m) f_{v}(v) N\left(\omega \approx m \omega_{s p}, k\right)=2 \gamma_{s}\left(\omega \approx m \omega_{s p}, k\right) N\left(\omega \approx m \omega_{s p}, k\right),
\end{aligned}
$$

where the Einstein coefficient $A_{S}(m)$ is given in Eq. (21), and we have neglected the Compton recoil terms. In Eq. (44), when $m=+1, m_{s}=+12$; when $m=-1, m_{s}=-1 / 2$; and $\gamma_{s}$ is the damping rate of the spin-flip excitations. The optical depth of these spin-flip excitations may be written

$$
\tau_{s}\left(\omega \approx m \omega_{s p}, k\right)=\int \mathrm{dl} \cdot\left(\frac{\gamma_{s}}{V_{g s}}\right),
$$

where $\mathrm{dl}$ and $\mathrm{V}_{\mathrm{gs}}=\partial \omega / \partial \mathrm{k}$ are the incremental path length and the group velocity, respectively, of the spin-flip excitations in the background medium under study. Since for frequencies $\omega$ which are less than the lower hybrid 24,30 frequency $\omega_{\text {LH }}$ of the background deuterium plasma the only allowed electromagnetic wave in this background medium is the hydromagnetic Alfven waves with $\omega \approx k V_{A}$, t seems reasonable here to take $V_{g s}$ as equal to $V_{A}$ and not as it's free space value of $c$. The physical reason for this is that these spin-flip excitations are (in the view of the conventional Bloch's equations for spin-flip resonance ${ }^{33}$ ) indeed either right or left circularly polarized 
transverse electromagnetic waves which are commensurable to the allowed Alfven waves of the background medium.

Here again, at the radiative steady state the spontaneous emission exactly balances the absorption minus the induced emission, i.e., the net rate of increase of the spin-flip excitations as given by Eq. (41) vanishes. Then the radiation temperature for these spin-flip excitations may be defined as

$$
\left(\frac{k T_{s r}}{\hbar \omega}\right)=\frac{\left[d N\left(\omega \approx m \omega_{s p}, k\right) / d t\right]_{s e m}}{\left[2 \gamma\left(\omega \approx m \omega_{s p}, k\right)\right]} .
$$

If $F_{s}(m)=\left[f_{s}\left(m_{s}\right)-f_{s}\left(-m_{s}\right)\right]>0, \gamma>0$, and the system will reach a radiative steady state with a finite value for the spin-flip excitation energy density $\varepsilon\left(\omega \approx m \omega_{s p}, k\right)=$ $k T_{s r}\left(\omega \approx m \omega_{s p}, k\right)=\hbar \omega N\left(\omega \approx m \omega_{s p}, k\right) / L^{3}$. At marginal stability $F_{S}(m)=\left[f_{S}\left(m_{s}\right)\right.$ $\left.f_{S}\left(-m_{S}\right)\right]=0$, i.e., the populations of the spin-up and the spin-down states become equal, $\gamma=0$, the steady state wave energy density and the radiation temperature tends to infinity. When $F_{S}(m)<0$, the population is inverted, and we have a spin-flip laser or maser which we will again examine in a later section.

\section{Evaluation of Optical Depths and Radiation Temperatures for Various Distributions}

In this section we wish to carry out the explicit evaluation of the optical depths $\tau$ and the corresponding radiation temperatures $T_{r}$ both for the cyclotron harmonic waves with the various distribution functions mentioned earlier and for the spin-flip excitations with the thermodynamic equilibrium distribution of Eqs. (23) and (24). Let us first examine the case of cyclotron harmonic fast Alfven waves. 


\section{Case 1: Cyclotron Harmonic Waves with $\theta=\pi / 2$}

Since the optical depth $\tau$ will take it's largest value for $\theta=\pi / 2$, i.e., $k=k_{\perp}$ and $k_{\|}=0$, for the purposes of our estimate we will only examine this case and the case of $\theta \approx \pi / 2$ here. Our main purpose in studying the case of $\theta \approx \pi / 2$ is to examine the effects of finite value of $k_{\|}$. Then on making use of Eqs. (3), (4) and (28) in Eq. (39) and carrying out two integration by parts (one over $d v_{\perp}$ and one over $\left.d v_{\|}\right)$, we obtain

$$
2 \gamma\left(\omega \approx m \omega_{c i}, k_{\perp}\right) \approx\left(\frac{\pi \omega_{p i}^{2}}{\mu^{2}}\right)\left\{\frac{S_{m}^{-1} \partial\left[S_{m}^{\prime} J_{m}^{\prime}\left(S_{m}\right)\right]^{2}}{\partial S_{m}}\right\} \delta\left(\omega-m \omega_{c i}\right)
$$

for the monoenergetic velocity space ring distribution of Eq. (28). Here $\mu^{2}=(c k / \omega)^{2} \approx\left(c / V_{A}\right)^{2} \approx C, S_{m}=\left(m V_{i} / V_{A}\right), \omega_{p i}=\left(4 \pi n_{i} q_{i}^{2} / M_{i}\right)^{1 / 2}$ is the ion plasma frequency, and the prime in the Bessel function denotes the derivative with respect to the argument. Since for the tokamak discharge $m \omega_{c i}=m q_{i} B / M_{i} c \propto R^{-1}, d \omega=-d\left(m \omega_{c i}\right)=\left(m \omega_{c i} / R_{c}\right) d R$, where $R$ is the major radius of the torus and $R_{c}$ is the major radius of the cyclotron resonance layer. Thus, on making use of Eq. (47) in Eq. (40) we obtain the optical depth $\tau$ of this cyclotron resonance layer in the tokamak discharges as

$$
\tau\left(\omega=m \omega_{c i}, k_{\perp}\right)=\left(\frac{R_{c}}{m \omega_{c i}}\right) \int d \omega\left(\frac{\gamma}{V_{A}}\right)=\left(\frac{\pi R_{c} \omega_{p i}^{2}}{2 \mu^{2} m \omega_{c i} V_{A}}\right)\left(\frac{S_{m}^{-1} \partial\left[S_{m} J_{m}^{\prime}\left(S_{m}\right)\right]^{2}}{\partial S_{m}}\right)
$$

Similarly, from Eqs. (3), (4), (28), and (42) we obtain the radiation temperature $T_{r}$ for these cyclotron harmonic waves appropriate to the velocity space ring distribution of Eq. (28) as

$$
k T_{r}\left(\omega \approx m \omega_{c i}, k_{\perp}\right)=\frac{\left(M_{i} V_{A}^{2} / m^{2}\right)\left[S_{m} J_{m}^{\prime}\left(S_{m}\right)\right]^{2}}{S_{m}^{-1} \partial\left[S_{m} J_{m}^{\prime}\left(S_{m}\right)\right]^{2} \partial S_{m}}
$$

For the monoenergetic isotropic spherical shell distribution of Eq. (29) we obtain

$$
2 \gamma\left(\omega=m \omega_{c i}, k_{\perp}\right) \approx\left(\frac{\pi \omega_{p i}^{2}}{\mu^{2}}\right)\left(\frac{S_{m}^{-1} \partial\left\{\left[S_{m} J_{m}^{\prime}\left(S_{m}\right)\right]^{2}+\left[S_{m} J_{m}\left(S_{m}\right)\right]^{2} / 2\right\}}{\partial S_{m}}\right) \delta\left(\omega-m \omega_{c i}\right)
$$

for the damping rate, 


$$
\tau\left(\omega=m \omega_{c i}, k_{\perp}\right)=\left(\frac{\pi R_{c} \omega_{p i}^{2}}{2 \mu^{2} m \omega_{c i} V_{A}}\right)\left(\frac{S_{m}^{-1} \partial\left[\left[S_{m} J_{m}^{\prime}\left(S_{m}\right)\right]^{2}+\left[S_{m} J_{m}\left(S_{m}\right)\right]^{2} / 2\right]}{\partial S_{m}}\right)
$$

for the optical depth, and

$$
K T_{r}\left(\omega \approx m \omega_{c i}, k_{\perp}\right) \sim\left(\frac{M_{i} V_{A}^{2}}{m^{2}}\right) \frac{\left[S_{m} J_{m}^{\prime}\left(S_{m}\right)\right]^{2}+\left[S_{m} J_{m}\left(S_{m}\right)\right]^{2} / 2}{\left.\partial\left[S_{m} J_{m}^{\prime}\left(S_{m}\right)\right]^{2}+\left[S_{m} J_{m}\left(S_{m}\right)\right]^{2} / 2\right) / \partial S_{m}}
$$

for the radiation temperature of these cyclotron harmonic waves.

For the anisotropic Maxwell-Boltzmann velocity space distribution of Eqs. (30) and (31), it is relatively easy to show that

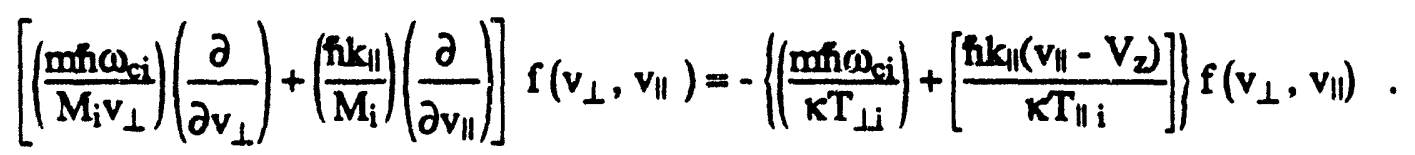

Thus, on making use of Eqs. (3), (4), (14), (30-36), and (j3) in Eqs. (39), (40), and (42) we obtain

$$
2 \gamma\left(\omega=m \omega_{c i}, k_{\perp}\right)=\left(\frac{\pi \omega_{p i}^{2}}{\mu^{2}}\right)\left(\frac{V_{A}^{2} M_{i}}{m^{2} K T_{\perp i}}\right)<G_{m}>\delta\left(\omega-m \omega_{c i}\right)
$$

for the damping rate,

$$
\tau\left(\omega=m \omega_{c i}, k_{\perp}\right)=\left(\frac{\pi R_{c} \omega_{p i}^{2}}{2 \mu^{2} m \omega_{c i} V_{A}}\right)\left(\frac{V_{A}^{2} M_{i}}{m^{2} K T_{\perp i}}\right)<G_{m}>
$$

for the optical depih, and

$\kappa T_{r}\left(\omega=m \omega_{c i}, k_{\perp}\right)=\left[\frac{(\hbar \omega)\left(\pi \omega_{p i}^{2} M_{i} / \mu^{2}\right)\left(V_{A} m\right)^{2}<G_{m}>\delta\left(\omega-m \omega_{c i}\right)}{\left(m \hbar \omega_{c i} / K T_{\perp j}\right)\left(\pi \omega_{p i}^{2} M_{i} / \mu^{2}\right)\left(V_{A} m\right)^{2}<G_{m}>\delta\left(\omega-m \omega_{c i}\right)}\right]=K T_{\perp j}$

for the radiation temperature, respectively, for the Maxwellian distribution of Eqs. (30) and (31). Here the $\left\langle\mathrm{G}_{\mathrm{m}}\right\rangle$ is given by Eq. (36). It is interesting to note from Eq. (56) that at thermodynamic equilibrium, the radiation temperature $T_{r}=T_{\perp i}$ implying that the ion cyclotron harmonic radiation 
field is in thermodynamic equilibrium with the radiating ions so as to satisfy the classical equipartition theorem as it should. According to the classical equipartition theorem 14 of course the energy per degree of freedom is $\mathrm{kT} / 2$. The radiation field has two degrees of freedom, one corresponding to its kinetic energy and the other to its potential energy, so that its energy density $=2\left(k T_{d} / 2\right)=k T_{r}$. This radiation is due to the perpendicular cyclotron motion of the particles which also has two degrees of freedom, one corresponding to its motion along the $x$ axis and the other to its motion along the $y$ axis, so that its average kinetic energy is $2\left(\kappa T_{\perp i} / 2\right)=\kappa T_{\perp j}$.

\section{Case 2: Cyclotron Harmonic Waves with $\theta \approx \pi / 2$}

In this case it can be seen from Eqs. (3) and (4) that for the monoenergetic velocity space ring distribution of Eq. (28) the damping rate $\gamma$, the optical depth $\tau$, and the radiation temperature $T_{\mathrm{r}}$ are given by Eqs. (47), (48), and (49), respectively, with the following replacement: $\left[S_{m} J_{m}^{\prime}\left(S_{m}\right)\right]^{2}$ is now replaced by $\left\{\left[S_{m} J_{m}^{\prime}\left(S_{m}\right)\right]^{2}+\left[(m \cos \theta / \sin \theta) J_{m}\left(S_{m}\right)\right]^{2}\right\}$. Similarly, for the isotropic velocity space spherical shell distribution of Eq. (29) these variables $\gamma, \tau$, and $T_{r}$ are given by the Eqs. (50), (51), and (52), respectively, with the following replacement: $\left[S_{m} J_{m}\left(S_{m}\right)\right]^{2}$ is now replaced by $\left\{\left[\left(m \cos \theta-2^{-1 / 2} S_{m}\right) / \sin \theta\right] J_{m}\left(S_{m}\right)\right\}^{2}$. Here again for the anisotropic Maxwell-Boltzmann velocity space distribution of Eqs. (30) and (31), it can be shown that $\gamma, \tau$, and $T_{r}$ are given by Eqs. (54), (55), and (56), respectively, with the following replacement: $\left\langle G_{m}\right\rangle$ is now replaced by $<\widetilde{G}_{m}>$ of Eq. (37) and $\delta\left(\omega-m \omega_{c i}\right)$ is now replaced by $\left\{(k \cos \theta)^{-1} f_{\|}\left[v_{\|}=\left(\omega-m \omega_{c i}\right) / k \cos \theta\right]\right\}$. It may be noted from Eq. (31) that when $(k \cos \theta) \rightarrow 0$, the factor $\left\{(k \cos \theta)^{-1} f_{\|}\left[v_{\|}=\left(\omega-m \omega_{c i}\right) / k \cos \theta\right]\right\} \rightarrow \delta\left(\omega-m \omega_{c i}\right)$ and hence the result of Eq. (55) is valid for $\theta \approx \pi / 2$ if we take $\left\langle\widetilde{G}_{m}\right\rangle \approx\left\langle G_{m}\right\rangle$ after the frequency integration.

In a similar way from the results found above, one can obtain closed form expressions for the damping rate $\gamma$, the optical depth $\tau$, and the radiation temperature $T_{r}$ for the cases of $\theta=0$ and $\theta=0$. As we stated earlier, the largest values of these variables occur for the case of $\theta=\pi / 2$ which we have discussed in detail here. This follows because the cyclotron harmonic emission and absorption at $\omega \approx m \omega_{c}$ for $m \geq 2$ occur only due to the finite value of $k_{\perp}$, and the Bessel functions $J_{m}\left(k_{\perp} v_{\perp} / \omega_{c i}\right)$ take large values when $k_{\perp}=m \omega_{c i} / v_{\perp}$. We have also discussed the case of $\theta \approx \pi / 2$ in order to illustrate the effects of finite $k_{\|}$via the cyciotron overstability type terms. Indeed, from the formulae given here one can obtain the closed form expressions for all these parameters $\gamma, \tau$, and $T_{r}$ for any arbitrary angle $\theta$. 


\section{Case 3: For Spin-Flip Excitations}

-r the case of fusion product proton spin-flip excitations, on making use of Eqs. (21), (23) and (24) in Eqs. (44), (45), and (46) we obtain

$$
\begin{aligned}
2 \gamma_{s}\left(\omega \approx m \omega_{s p}, k\right) \approx & {\left[\left(\frac{\pi \omega_{p i}^{2}}{\mu^{2}}\right)\left(\frac{g_{p}^{2}}{8}\right)\left(\frac{\hbar k}{M_{p} V_{A}}\right) F_{s}(m)\right] } \\
& \left\{|k|^{-1}\left(\frac{M_{p}}{2 \pi K^{\prime} T_{v}}\right)^{1 / 2} \exp \left[\frac{-M_{p}\left(\omega-m \omega_{s p}\right)^{2}}{|k|^{2} 2 K T_{v}}\right]\right\}
\end{aligned}
$$

for their damping rate,

$$
\tau\left(\omega \approx m \omega_{s p}, k\right)=\left(\frac{\pi R_{s} \omega_{p i}^{2}}{2 \mu^{2} \omega_{s p} V_{A}}\right)\left(\frac{g_{p}^{2}}{8}\right)\left(\frac{\hbar k}{M_{p} V_{A}}\right) F_{s}(m)
$$

for their optical depth in tokamak B fields, and

$$
\begin{aligned}
k T_{r}\left(\omega \approx m \omega_{s p}, k\right)=\left(\hbar \omega_{s p}\right) f_{s}\left(m_{s}\right)\left[F_{s}(m)\right]^{-1}=\left(\hbar \omega_{s p}\right) f_{s}\left(m_{s}\right)\left[f_{s}\left(m_{s}\right)-f_{s}\left(-m_{s}\right)\right]^{-1} \\
=\left(\hbar \omega_{s p}\right)\left[1-\frac{f_{s}\left(-m_{8}\right)}{f_{s}\left(m_{s}\right)}\right]^{-1}=\left(\hbar \omega_{s p}\right)\left(\frac{2 k T_{s}}{2 \hbar \omega_{s p}}\right)=k T_{s} \text { for } \hbar \omega_{s p} \ll k T_{s},
\end{aligned}
$$

for the radiation temperature, respectively. In Eq. (57) we have written $\omega / k \approx V_{A}$, i.e., $\left(\hbar k^{2} / M_{p} \omega\right) \approx\left(\hbar k / M_{p} V_{A}\right)=\left(\hbar \omega / M_{p} V_{A}^{2}\right)$. In Eq. (58) we have used the fact that the tokamak confining magnetic field $B \propto R^{-1}$ and the major radius of the spin-flip resonance layer is $\mathbf{R}_{\mathbf{s}}$. In Eq. (59) we have divided both the numerator and the denominator by the common factor $\left\{\left(\pi \omega_{p i}^{2} / \mu^{2}\right)\left(g_{p}^{2} / 8\right)\left(\pi k^{2} / M_{p} c \omega\right)|k|^{-1} f_{v}\left[|v|=\left(\omega-\omega_{s p}\right) /|k|\right]\right\}$. Note that in Eq. (57), when $T_{v} \rightarrow 0$ the factor $\left(|k|^{-1}\left(M_{p} / 2 \pi k T_{v}\right)^{1 / 2} \exp \left[-M_{p}\left(\omega-m \omega_{s p}\right)^{2} /|k|^{2} 2 k T_{v}\right\} \rightarrow \delta\left(\omega-\omega_{s p}\right)\right.$; and for $B \propto R^{-1}, d R=-\left(R d \omega_{s p} / \omega_{s p}\right)=\left(R d \omega / \omega_{s p}\right)$. Thus the result of Eq. (58) follows trivially from Eqs. (45) and (57) since $V_{g s}=V_{A}$. Here again we see from Eq. (59) that the spin-flip excitations obey the classical equipartition theorem at thermodynamic equilibrium as they should of course. 


\section{CONDITIONS FOR RADIATIVE INSTABILITY AND/OR OVERSTABILITY}

In this section we wish to examine briefly the physical conditions for which the system under study can become unstable both for the cyclotron harmonic emission and for the spin-flip emission.

\section{Case 1: For Cyclotron Harmonic Emission}

It is well known that in the linearized theory the spontaneous emission is independent of the intensity of the radiation field present in the system while both the induced emission and the direct absorption depend on the intensity of the radiation field the system is bathed in. In Dirac's quantum theory of emission and absorption of a single photon, 34 this linearized theory corresponds to the use of the Fermi golden rule approximation in addition to the usual random phase approximation in the conventional time dependent quantum perturbation theory. 35,36 Thus it is seen from Eqs. (39) and (41) that the most general condition for linear radiative instability is that

$$
\left[\frac{\mathrm{dN}(\omega, k)}{\mathrm{dt}}\right]=\left[\frac{\mathrm{dN}(\omega, k)}{\mathrm{dt}}\right]_{\mathrm{sem}}-2 \gamma(\omega, k) \mathrm{N}(\omega, k) \geq 0 .
$$

That is, for linear radiative instability in quantum nonequilibrium statistical mechanics we require that the spontaneous emission exceeds the absorption minus the induced emission, i.e., $2 \gamma(\omega, k) N(\omega, k) \leq[d N(\omega, k) / d t]_{s e m}$. In classical plasma kinetic theory it is extremely difficult if not impossible to calculate this spontaneous emission, and thus one usually assumes that the intensity or the energy density of the radiation field $\varepsilon(\omega, k)=\hbar \omega N(\omega, k) / L^{3}$ is large enough so that one can always neglect the spontaneous emission. Thus, for linear radiative instability in classical plasma kinetic theory we will assume that $[\mathrm{dN}(\omega, \mathrm{k}) / \mathrm{dt}]_{\text {sem }}=0$ and we only require that $\gamma(\omega, k) \leq 0$, i.e., we only require that the induced emission exceeds the absorption. Under these conditions the system will behare as an ion cyclotron harmonic laser or maser for the fast Alfven waves with $\omega=m \omega_{c i}$. Thus from Eq. (39) the classical instability and/or the overstability condition necessary for the cyclotron harmonic laser or maser action may be written

$$
\begin{aligned}
\int d v_{\perp}\left(2 \pi v_{\perp}\right) \int d v_{\|}\left[L^{3} n_{i} A(m)\right]\left[\left(\frac{m \omega_{c i}}{M_{i} v_{\perp}}\right)\left(\frac{\partial}{\partial v_{\perp}}\right)\right. & \left.+\left(\frac{\hbar k_{\|}}{M_{i}}\right)\left(\frac{\partial}{\partial v_{\| 1}}\right)\right] f\left(v_{\perp}, v_{\| l}\right) \\
& =-2 \gamma\left(\omega \approx m \omega_{c i}, k\right) \geq 0 .
\end{aligned}
$$


If the total distribution function $f\left(v_{\perp}, v_{\|}\right)$can be decomposed as a product $f_{\perp}\left(v_{\perp}\right) f_{\|}\left(v_{\|}\right)$, then by doing an integration by parts over $d v_{\perp}$ in Eq. (61) it is relatively easy to see that the sign of the net result for $\gamma$ is not at all sensitive to the sign of the slope $\left[\partial f_{\perp}\left(v_{\perp}\right) / \partial v_{\perp}\right]$ anywhere in the range $0 \leq v_{\perp} \leq \infty$, since $\int_{0}^{\infty} d v_{\perp}\left(2 \pi v_{\perp}\right) f_{\perp}\left(v_{\perp}\right)=1$. The reason for this is that the $\delta$ functions of the $A(m)$ 's of Eqs. (3) and (4) do not select or favor any particular value of $v_{\perp}$ as a particle-wave resonance value in $f_{\perp}\left(v_{\perp}\right)$. These $\delta$ functions do however imply the particle-wave resonance in the $f_{\|}\left(v_{\|}\right)$at $v_{\|}=\left(\omega-m \omega_{c i}\right) / k_{\| l}$ and hence in Eq. (61) the only term whose sign can depend on the slope of the distribution function is the term $\left(\hbar k_{\|} / M_{i}\right)\left[\partial f_{\|}\left(v_{\|}\right) / \partial v_{\| l}\right]$. Thus, for example, if we use the anisotropic Maxwell-Boltzmann distributions of Eqs. (30) and (31) or equivalently if we make use of Eq. (53) in Eq. (61), we may write the necessary condition for the cyclotron harmonic maser as

$$
\left[\left(\frac{m \omega_{c i}}{T_{\perp i}}\right)+\frac{\left(\omega-m \omega_{c i}-k_{\|} V_{z}\right)}{T_{\| i}}\right] \leq 0 .
$$

This is the familiar cyclotron overstability condition as found in Stix. ${ }^{24}$ For $\omega \approx m \omega_{\text {ci }}$, we see from Eq. (62) that for a cyclotron harmonic maser we need a large enough drift velocity $V_{z}$ such that $V_{z} \geq\left(m \omega_{c i} T_{\| i} / k_{\|} T_{\perp i}\right)=\left(V_{A} / \cos \theta\right)\left(T_{\| i} / T_{L i}\right)$. For example, for $T_{\perp i}=T_{\| i}, \omega=m \omega_{c i}$ and $k_{\|} \approx k$ then for maser action $V_{2} \geq \omega / k \sim V_{A}$. For $k_{\|}=0$, Eq. (62) yields $\left(\omega / m_{c_{i}}\right)$ $\leq\left(1-T_{\| i} / T_{\perp i}\right)$. This in turn implies that $\omega \leq m \omega_{c i}$ and $T_{\perp i} \geq T_{i l i}$. It may be pointed out that the above analysis also applies equally well to the slow, short wave length electrostatic ion Bernstein waves (ESIBW). For ESIBW, $k \approx k_{\perp}$ and the phase velocity $\omega / k \approx m \omega_{c i} / k_{\perp}=m \omega_{c i} \rho_{i} / k_{\perp} \rho_{i}=m\left(2 \kappa T_{\perp i} / M_{i}\right)^{1 / 2}\left(k_{\perp} \rho_{i}\right)^{-1}=m\left(2 \kappa T_{\perp j} / M_{i}\right)^{1 / 2}$ since $\left(k_{\perp} \rho_{i}\right)$ is of order unity for IBW of interest. In the literature Harris, 37 Davidson and $W u, 38$ Gaffey, Thompson, and Liu, 39 and others 40 have examined somewhat similar plasma wave instabilities and/or negative energy waves.

\section{Case 2. For Spin-Flip Emission}

Here again the most general condition for linear radiative instability is the same as that given by Eq. (60). That is, in quantum nonequilibrium statistical mechanics we require that the spontaneous emission of spin-flip excitations exceeds the absorption minus the induced emission for the linear radiative instability, i.e., $2 \gamma_{s}\left(\omega \approx \omega_{\text {sp }}, k\right) N\left(\omega \approx \omega_{s p}, k\right)$ $\leq\left[\mathrm{dN}\left(\omega \approx \omega_{\mathrm{sp}}, \mathrm{k}\right) / \mathrm{dt}\right]_{\mathrm{sem}}$. Just as in the classical plasma kinetic theory we will assume that the energy density of the radiation field due to these spin-flip excitations is large enough so that we can always neglect the spontaneous emission. Thus the necessary condition for the spin-flip laser or 
maser action is that $\gamma_{s}(\omega, k) \leq 0$, i.e., we require that the induced emission exceed the absorption. Thus for example from Eq. (44) we see that $\gamma \leq 0$ if and only if

$$
F_{s}(m)=\left[f_{s}\left(m_{s}\right)-f_{s}\left(-m_{s}\right)\right] \geq 0
$$

That is, for a spin-flip maser or laser the statistical population of the spin-up and the spin-down states must be inverted. 41,42 However, in Eq. (44) we have neglected the Compton recoil contribution to $\gamma_{s}$. Thus, from Eq. (74) of Ref. 28, retaining this Compton recoil contribution to $\gamma_{s}$, we obtain

$$
\begin{aligned}
2 \gamma_{s}(\omega, k) & =\frac{[d N(\omega, k) / d t]_{a b}-[d N(\omega, k) / d t]_{i e m}}{N(\omega, k)} \\
& =-\int d v\left[L^{3} n_{p} A_{s}(m)\right]\left[f_{s}\left(m_{s}\right) f_{v}\left(v+\frac{\hbar k}{M_{p}}\right)-f_{s}\left(-m_{s}\right) f_{v}(v)\right] \\
& \approx-\int d v\left[L^{3} n_{p} A_{s}(m)\right]\left[F_{s}(m)+f_{s}\left(m_{s}\right)\left(\frac{\hbar k}{M_{p}}\right) \cdot \nabla_{v}\right] f_{v}(v),
\end{aligned}
$$

where $F_{s}(m)=\left[f_{s}\left(m_{s}\right)-f_{s}\left(-m_{s}\right)\right]$, and we have Taylor series expanded $f_{v}\left(v+\hbar k / M_{p}\right) \approx f_{v}(v)+\left(\hbar k / M_{p}\right) \cdot \nabla_{v} f_{v}(v)+\cdots=f_{v}(v)+\left(\hbar k / M_{p}\right) \cdot\left[\partial f_{v}(v) / \partial v\right]+\cdots$. From Eq. (64) we see that taking account of the Compton recoil effects, the necessary condition for a spin-flip laser or maser may be written

$$
\left[F_{s}(m)+f_{s}\left(m_{s}\right)\left(\frac{\hbar k}{M_{p}}\right) \cdot \nabla_{v}\right] f_{v}(v) \geq 0
$$

If we now make use of Eqs. (23) and (24) in Eq. (65), the necessary condition for spin-flip overstability or instability becomes

$$
\left[\left(\frac{m \omega_{s p}}{k T_{s}}\right)+\left(\frac{\omega-m \omega_{s p}-k \cdot V_{d}}{k T_{v}}\right)\right] \leq 0 .
$$


It is instructive to compare the spin-flip overstability or spin-flip maser condition of Eq. (66) with the corresponding cyclotron harmonic overstability or the cyclotron harmonic maser condition of Eq. (62). The spin temperature $\Gamma_{S}$ takes the role of the perpendicular ion temperature $T_{\perp i}$ while the kinetic translational temperature $T_{V}$ plays the same role as the parallel ion temperature $T_{\| i}$ and $k \cdot V_{d}$ replaces $k_{\|} V_{z}$. Further, for $\omega \approx m \omega_{s p}$ we see from Eq. (66) that for a spin-flip laser or maser we need a large enough drift velocity $V_{d}$ such that $\left(k \cdot V_{d}\right) \geq\left(m \omega_{s p} T_{v} / T_{s}\right)$. For $\left(k \cdot V_{d}\right)=0$, Eq. (66) yields $\left|\left(\omega / m \omega_{s p}\right)\right| \leq\left(1-T_{v} / T_{s}\right)$. This in turn implies that $|\omega| \leq\left|m \omega_{s p}\right|$ and $T_{s} \geq T_{v}$.

\section{QUASILINEAR AND/OR NONLINEAR RADIATIVE STEADY STATE}

In Sec. III we have examined the linearized theory of the radiative steady state. In this linearized theory we have made the intrinsic assumption that the particle distribution function and/or the statistical population of the quantum states is completely unaffected during the entire time evolution of the total system of particles and their radiation field towards its linear radiative steady state. That is, the linearized steady state is determined purely by the steady state solution of the photon master equation [i.e., our Eq. (41)] of nonequilibrium statistical mechanics assuming of course a fixed given particle distribution function and the sufficiency of the Fermi golden rule approximation in addition to the usual random phase approximation to the standard time dependent quanturn perturbation theory. However, according to the rules of nonequilibrium statistical mechanics the time evolution of the coupled photon-particle system is in general determined by a coupled set of master equations, one [such as for example our Eq. (41)] describing the time evolution of the radiation field or photon (i.e., the field oscillators) distribution function and the other describing the time evolution of the emitting and absorbing particle (i.e., the particle oscillators) distribution function. This is because the processes of emission and absorption of photons occur only as a consequence of the emitting or absorbing particle changing its statistical state. Thus the time evolution of the photon distribution function will depend on the instantaneous value of the particle distribution function and the time evolution of the particle distribution function will in turn depend on the instantaneous value of the photon distribution function. It is this coupling between the particle oscillators and the field oscillators which we wish to examine in this section.

If we use the Fermi golden rule approximation to obtain the Einstein A and B coefficients, then this coupled pair of equations describing the photon-particle system form the basis of the conventional quasilinear theory of the radiative steady state. We will see that in this quasilinear approximation the master equation for the particle oscillators will reduce in the classical limit to a 
Fokker-Planck equation in which there will appear the usual velocity space diffusion and dynamical friction terms. The diffusion term will depend on the instantaneous value of the radiation field intensity since it is a consequence of induced emission minus absorption, while the dynamical friction term is independent of the field intensity since it is a consequence of spontaneous emission. In the conventional classical plasma kinetic theory approach it is extremely difficult if not impossible to derive this dynamical friction term.

However, this Fermi golden rule approximation only makes use of the first term of the series solution of the integral equation for the quantum mechanical transition probabilities due to Heitler and $\mathrm{Ma} .35,36$ In the modern formal quantum theory of scattering phenomeia in quantum electrodynamics (QED) this Heitler-Ma integral equation formalism is sometimes referred to as the Dyson's $S$ and/or T matrix formalism. 34,43 Basically, according to Heitler and Ma the quantum mechanical transition probability $j(f ; i)$ from the initial state $l i>$ of energy $E_{i}$ to a final state $\mid f>$ of energy $E_{f}$ due to an interaction Hamiltonian $T_{\text {int }}$ may be written

$$
j(f ; i)=\left(\frac{2 \pi}{h}\right)|<f| T|i>|^{2} \delta\left(E_{f}-E_{i}\right),
$$

where

$$
\langle\mathrm{f}|\mathrm{T}| \mathrm{i}\rangle=\left\langle\mathrm{f}\left|\mathrm{T}_{\mathrm{int}}\right| \mathrm{i}\right\rangle+\Sigma_{\mathrm{f}^{\prime} \neq \mathrm{i}}\left\{\frac{\left.\left[<\mathrm{f}\left|\mathrm{T}_{\mathrm{int}}\right| \mathrm{f}^{\prime}><\mathrm{f}^{\prime}|\mathrm{T}| \mathrm{i}\right\rangle\right]}{\left(E_{\mathrm{i}}-E_{\mathrm{f}^{\prime}}\right)}\right\},
$$

where $\mid f^{\prime}>$ is an intermediate state of energy $E_{f}$ '. The series solution of the integral Eq. (68) may be written

$$
\begin{aligned}
& \left.<\mathrm{f}|\mathrm{T}| \mathrm{i}\rangle=<\mathrm{f}\left|\mathrm{T}_{\mathrm{int}}\right| \mathrm{i}\right\rangle+\Sigma_{\mathrm{f} \neq \mathrm{i}}\left\{\frac{\left[\left\langle\mathrm{f}\left|\mathrm{T}_{\text {int }}\right| \mathrm{f}\right\rangle\left\langle\mathrm{f}^{\prime}\left|\mathrm{T}_{\mathrm{int}}\right| \mathrm{i}\right\rangle\right]}{\left(\mathrm{E}_{\mathrm{i}}-\mathrm{E}_{\mathrm{f}^{\prime}}\right)}\right\} \\
& +\Sigma_{\mathrm{f}} \neq \Sigma_{\mathrm{f}} \Sigma_{\mathrm{f}} \neq \mathrm{i}\left\{\frac{\left.\left[<\mathrm{f}\left|\mathrm{T}_{\text {int }}\right| \mathrm{f}^{\prime}><\mathrm{f}\left|\mathrm{T}_{\text {int }}\right| \mathrm{f} "><\mathrm{f}{ }^{\prime \prime}\left|\mathrm{T}_{\text {int }}\right| \mathrm{i}\right\rangle\right]}{\left[\left(E_{\mathrm{i}}-\mathrm{E}_{\mathrm{f}}\right)\left(\mathrm{E}_{\mathrm{i}}-E_{\mathrm{f}}{ }^{\prime \prime}\right)\right]}\right\}+\cdots,
\end{aligned}
$$

where $\mid f^{\prime \prime}>$ is another intermediate state of energy $E_{f}$ ". Furthermore, our Einstein's coefficients $A(m)^{\prime} s$ of Eqs. (3) and (4) are due to the interaction Hamiltonian $\mathrm{H}_{\text {int }}=-\left(\mathrm{q}_{\mathrm{i}} \mathbf{p} \cdot \mathbf{A} / \mathrm{M}_{\mathrm{i}} \mathrm{c}\right)=-\left(\mathrm{q}_{\mathrm{i}} \mathbf{v} \cdot \mathbf{A} / \mathrm{c}\right)$ which is responsible for transitions in which only one light quantum is involved. Here $\mathbf{A}$ is the vector potential of the transverse radiation field in the box of 
volume $\mathrm{L}^{3}$ expressed in terms of its creation and annihilation operators. However, for the higher order Heitler-Ma terms of Eq. (69) we must not only include the two-photon interaction Hamiltonian $\mathrm{H}^{\prime}{ }_{\text {int }}=\left(\mathrm{q}_{\mathrm{i}}^{2} / 2 \mathrm{M}_{\mathrm{i}} \mathrm{c}^{2}\right) \mathbf{A}^{2}$ that is responsible for scattering of transverse photons but also include the interaction due to the longitudinal and scalar photons. ${ }^{20}$ Then these higher order Heitler-Ma terms will yield all the nonlinear processes 36 such as the nonlinear Landau damping, nonlinear mode coupling, nonlinear decay interactions, linear and nonlinear mode conversion 20 (i.e., absorption of a transverse or a scalar photon and the subsequent emission of a longitudinal or transverse photon, respectively), etc. Thus, if we make use of the nonlinear Einstein A and B coefficients calculated with the full use of the Heitler-Ma time dependent perturbation theory, then the resultant coupled pair of master equations obtained by using the principle of detailed balance will in principle determine the nonlinear radiative steady state of the coupled particle-photon (both the transverse and longitudinal and scalar photons) system. It is not very clear to us whether or not the bounce frequency effects due to particle trapping, 44,45 the Karplus-Schwinger nonlinear resonance broadening, 46,41 and Dupree's 47 turbulent velocity space diffusion broadening are also embodied in this Heitler-Ma theory. 35,36

Although for the sake of completeness we have indicated the physical principles underlying the development of the full mathematical quantum theory of turbulence in sufficient detail so as to be physically instructive, pedagogically speaking, we will now only indicate the coupled set of quasilinear equations here. Further, we are here interested in the classical limit of this coupled pair of quasilinear equations. 48 On making use of Eq. (39) of Ref. 48 in Eqs. (16) and (17) of this reference we obtain

$$
\begin{aligned}
& {\left[\frac{\partial \varepsilon(\omega, k)}{\partial t}\right]_{m}=\int d v_{\perp}\left(2 \pi v_{\perp}\right) \int d v_{\|}\left[L^{3} n_{i} \hbar \omega A(m)\right]\left\{\left[\varepsilon(\omega, k) Q_{m}\right]+1\right\} f\left(v_{\perp}, v_{\| l}\right)} \\
& \quad=\left(\frac{\pi \omega_{p i}^{2} M_{i}}{\mu^{2}}\right)\left(\frac{V_{A}}{m}\right)^{2} \int d v_{\perp}\left(2 \pi v_{\perp}\right) \int d v_{\|} \widetilde{G}_{m}
\end{aligned}
$$

for the rate of growth of the energy density $\varepsilon(\omega, k)=\hbar \omega N(\omega, k) / L^{3}$ of the cyclotron harmonic Alfven waves near the m-th harmonic, and 


$$
\begin{gathered}
{\left[\frac{\partial f\left(v_{\perp}, v_{\|}\right)}{\partial t}\right]_{m}=\Sigma_{k}\left[\left(\frac{4 \pi^{2} q_{i}^{2}}{L^{3} \mu^{2}}\right)\left(\frac{V_{A}}{m}\right)^{2} \widetilde{G}_{m}\right]\left\{\left[\varepsilon(\omega, k) Q_{m} \delta\left(\omega-m \omega_{c i}-k_{\|} v_{\|}\right) Q_{m} f\left(v_{\perp} v_{\|}\right)\right]\right.} \\
\left.+\left[Q_{m} \delta\left(\omega-m \omega_{c i}-k_{\|} v_{\|}\right) f\left(v_{\perp}, v_{\| 1}\right)\right]\right\}=\int d \Omega \int d \omega\left[\left(\frac{\mu q_{1}^{2} \omega^{2}}{2 \pi c^{3}}\right)\left(\frac{v_{A}}{m}\right)^{2} \widetilde{G}_{m}\right] \\
\left\{\left[\varepsilon(\omega, k) Q_{m} \delta\left(\omega-m \omega_{c i}-k_{\|} v_{\|}\right) Q_{m} f\left(v_{\perp}, v_{\| l}\right)\right]+\left[Q_{m} \delta\left(\omega-m \omega_{c i}-k_{\|} v_{\|}\right) f\left(v_{\perp}, v_{\|}\right)\right]\right\}
\end{gathered}
$$

for the rate of change of the particle distribution function due to the emission and absorption of the $\mathrm{m}$-th cyclotron harmonic waves, where we have made use of Eq. (5), $\widetilde{\mathrm{G}}_{\mathrm{m}}$ is given in Eq. (17), and the linear diffierential operator

$$
\mathrm{Q}_{\mathrm{m}}=\left(\frac{\mathrm{m} \omega_{\mathrm{ci}}}{\omega}\right)\left[\left(\frac{1}{\mathrm{M}_{\mathrm{i}} \mathrm{v}_{\perp}}\right)\left(\frac{\partial}{\partial v_{\perp}}\right)+\left(\frac{k_{\|}}{\mathrm{M}_{\mathrm{i}} \mathrm{m} \omega_{\mathrm{ci}}}\right)\left(\frac{\partial}{\partial \mathrm{v}_{\|}}\right)\right] .
$$

The coupled set of classical quasilinear equations, Eqs. (70) and (71), for the wave energy density and the particle distribution function, respectively, provides a simple physical picture of the way the quasilinear steady state is established between the ions (i.e., the particle oscillators) and their cyclotron harmonic Alfven wave radiation field (i.e., the field oscillators). The rate of change of the wave energy density depends on the instantaneous value of the particle distribution function, and the rate of change of the particle distribution function in turn depends on the instantaneous value of the wave energy density. The first term of Eq. (70) represents the rate of damping (the so-called cyclotron damping; that is, the rate of absorption minus the rate of stimulated or induced emission) of these Alfven waves, while the second term of this equation gives the appropriate classical rate of spontaneous emission of the electromagnetic waves. It is seen that Eq. (71) is a Fokker-Planck equation whose first and second term represent a velocity space "diffusion" and "dynamical friction," respectively. This diffusion is proportional to the intensity of the radiation field $\varepsilon(\omega, \mathbf{k})$ and is a consequence of the difference between the induced emission and absorption while the dynamical friction is a consequence of the sporitaneous emission. Arunasalam ${ }^{49}$ had earlier made use of Eq. (71) to study the ion cyclotron resonance heating of plasmas and the associated longitudicul cooling which was first predicted using numerical simulation and basic thermodynamic arguments by Busnardo-Neto, Dawson, Kamimura, and Lin 50 and subsequently verified experimentally by Rapozo, de Assis and Busnardo-Neto. 51 Since there is increasing 
interest in ion cyclotron resonance heating (ICRH) of tokamak plasmas that are entering the reactor regime of operation, we wish to draw attention to the fact that an initially isotropic $\left(T_{\perp}=T_{11}\right)$ plasma will be forced into an anisotropic $\left(T_{\perp} \neq T_{\| 1}\right)$ state in ICRH, thus altering the ratio of trapped to circulating particles and the associated stability of tokamak plasmas. In particular, because the high temperature fusion plasmas are practically collisionless, it is extremely difficult (if not impossible) to avoid this $\left(T_{\perp}, T_{\| 1}\right)$ anisotropy in ICRH experiments. We should also point out that, in the literature, these types of coupled quasilinear equations were first derived by Pines and Schrieffer 52,19 for electron-plasmon and electron-phonon systems.

In a similar way from Eqs. (74), (75a), and (75b) of Ref. 28, the appropriate coupled pair of quasilinear equations for spin-flip emission and absorption may be written

$$
\begin{aligned}
& {\left[\frac{\partial \varepsilon(\omega, k)}{\partial t}\right]_{m} \approx \int d v\left[L^{3} n_{p} A_{s}(m)\right]\left\{\left[\varepsilon(\omega, k) F_{s}(m) f_{v}(v)\right]+(\hbar \omega) f_{s}\left(m_{s}\right) f_{v}(v)\right\}=\int d v\left[\frac{\pi \omega_{p i}^{2} g g_{p}^{2 \hbar \omega}}{8 M_{p} c^{2}}\right]} \\
& \left\{\left[\varepsilon(\omega, k) \delta\left(\omega-m \omega_{s p}-k \cdot v\right) F_{s}(m) f_{v}(v)\right]+\left[(\hbar \omega) \delta\left(\omega-m \omega_{s p}-k \cdot v\right) f_{s}\left(m_{s}\right) f_{v}(v)\right]\right\}
\end{aligned}
$$

for the rate of growth of the energy density $\varepsilon(\omega, k)=\hbar \omega_{\mathrm{sp}} N(\omega, k) / \mathrm{L}^{3}$ of the spin-flip excitations of $\omega \approx \mathrm{m} \omega_{\mathrm{sp}}= \pm \omega_{\mathrm{sp}}$,

$$
\begin{gathered}
{\left[\frac{\partial\left[F_{s}(m) f_{v}(v)\right]}{\partial t}\right]_{m}=-\Sigma_{k}\left(\frac{2}{L^{3} n_{p} \hbar \omega}\right)\left[\frac{\pi \omega_{p i}^{2} g_{p}^{2} \hbar \omega}{8 M_{p} c^{2}}\right]} \\
\left\{\left[\varepsilon(\omega, k) \delta\left(\omega-m \omega_{s p}-k \cdot v\right) F_{s}(m) f_{v}(v)\right]+\left[(\hbar \omega) \delta\left(\omega-m \omega_{s p}-k \cdot v\right) f_{s}\left(m_{s}\right) f_{v}(v)\right]\right\}
\end{gathered}
$$

for the rate of change of the difference in the population of the two spin states $F_{s}(m)=\left[f_{s}\left(m_{s}\right)-f_{s}\left(-m_{s}\right)\right]$, and $\left[\partial f_{v}(v) / \partial t\right]_{m} \approx 0$. Here we have assumed that $\hbar k \ll M_{p v}$ and hence neglected some Compton recoil effect terms. The $\Sigma_{k}$ in Eq. (74) is handled as an integral over $d \omega$ as illustrated in Eq. (5). The second terms in Eqs. (73) and (74), which are independent of $\varepsilon(\omega, \mathrm{k})$, are due to spontaneous emission and can usually be neglected in solving for the quasilinear steady state since the energy $\varepsilon^{\infty}(\omega, \mathrm{k})$ in the spin-flip excitations at this quasilinear steady state is considerably larger than their thermal equilibrium value of $\varepsilon^{\circ}(\omega, k) \approx \kappa T_{s}$.

A detailed study of the nonlinear stability of spin-flip excitations leading to the understanding of the spin-flip laser experiments of Patel and Shaw 53 has been presented elsewhere by 
Arunasalam. 41,42 Following the procedure outlined in these Refs, 41 and 42, one can show that Eqs. (73) and (74) can be combined to yield

$$
\frac{\partial\left[F_{s}(m)+\left(8 \mu^{3} \alpha \omega_{s p}^{2} / \pi \omega_{p i}^{2}\right)\left(\kappa T_{v} / M_{p} c^{2}\right)^{1 / 2}\left[d\left(\omega \approx m \omega_{s p}\right) / M_{p} c^{2}\right]\right\}}{\partial t} \approx 0
$$

where $\alpha=\left(q_{p}^{2} / h c\right) \approx(1 / 137)$. In deriving Eq. (75) we first neglect the spontaneous emission term of Eq. (74) and integrate Eq. (74) over $d v$ using the fact that $\left[\partial f_{v}(v) / \partial t\right]_{m}=0$. assume that $\int \mathrm{dv}$ and $\Sigma_{\mathrm{k}}$ processes commute, then make use of Eq. (73) neglecting the spontaneous emission term of Eq. (73) to substitute and eliminate $\int d v[]$ in terms of $[\partial \varepsilon(\omega, k) / \partial t]_{m}$, use Eq. (5) to go from $\Sigma_{k}\left[\right.$ ] to $\int d \omega[$ ], and in evaluating the integral over $d \omega$ obey the restrictions imposed by the Dirac $\delta$ function $\delta\left(\omega-m \omega_{s p}-k \cdot v\right)$. That is, the limits of the integral over $\omega$ are given by $\omega_{s p}\left(1-v_{0} / c\right) \leq \omega \leq \omega_{s p}\left(1+v_{0} / c\right)$, where the thermal speed $v_{0}=\left(\kappa T_{v} / M_{p}\right)^{1 / 2}$, and we make use of the approximation $\left[\left(1+v_{0} / c\right)^{3}-\left(1-v_{0} / c\right)^{3}\right] \approx 6 v_{0} / c$. It is clear from Eqs. (73-7.5) that $F_{s}(m) \approx 0$ at the quasilinear steady state, i.e., as $t \rightarrow \infty, F_{s}(m) \rightarrow F_{8} \infty=0$, $[\partial \varepsilon(\omega, k) / \partial t]_{m} \rightarrow 0$, and $\left[\partial F_{s}(m) f_{v}(v) / \partial t\right]_{m} \rightarrow 0$, where of course we have neglected the spontaneous emission terms which are independent of $\varepsilon(\omega, k)$. Thus at the quasilinear steady state the populations of the spin-up and the spin-down states are equal to each other and this is in accordance with the principle of minimum entropy production. 39,14

If at time $t=0$, the difference in the population of the two spin states $F_{s}(m)=F_{s}^{\circ}$, the spin-flip excitations energy $\varepsilon\left(\omega \approx m \omega_{\mathrm{sp}}\right) \approx \varepsilon^{0}$, and at the quasilinear steady state $F_{s}(m)=F_{s}^{\infty}=0$ and $\varepsilon\left(\omega \approx m \omega_{s p}\right) \approx \varepsilon^{\infty}$, then from Eq. (75) we obtain

$$
\varepsilon^{\infty}-\varepsilon^{\circ}=F_{s}^{o} M_{p} c^{2}\left(\frac{M_{p} c^{2}}{K T_{v}}\right)^{1 / 2}\left(\frac{\pi \omega_{p i}^{2}}{8 \mu^{3} \alpha \omega_{s p}^{2}}\right)
$$

where the thermal equilibrium initial value $\varepsilon^{0} \approx k T_{s} \approx k T_{v}$, and $\varepsilon^{\infty} \gg \varepsilon^{0}$. Hence, at the quasilinear steady state the spin-flip excitations energy density is enhanced by the factor 


$$
\begin{gathered}
\beta=\left[\frac{\left(\varepsilon^{\infty}-\varepsilon^{\circ}\right)}{\varepsilon^{0}}\right]=\left(\frac{\varepsilon^{\infty}}{\kappa T_{s}}\right)=F_{s}^{0}\left(\frac{M_{p} c^{2}}{\kappa T_{s}}\right)\left(\frac{M_{p} c^{2}}{\kappa T_{v}}\right)^{1 / 2}\left(\frac{\pi \omega_{p i}^{2}}{8 \mu^{3} \alpha \omega_{s p}^{2}}\right) \\
\approx F_{s}^{0}\left(\frac{M_{p} c^{2}}{\kappa T}\right)^{3 / 2}\left(\frac{\pi \omega_{p i}^{2}}{8 \mu^{3} \alpha \omega_{s p}^{2}}\right)
\end{gathered}
$$

above its black-body thermal equilibrium value of $k T_{s} \approx k T_{v}=k T$.

\section{SOME GENERAL COMMENTS ON INSTABILITIES}

In sections IV and V we have examined the conditions for radiative instability and/or overstability and the subsequent quasilinear and/or the nonlinear time evolution of such instabilities toward the corresponding quasilinear and/or the nonlinear radiative steady state. However, the instabilities discussed here are a consequence of the induced emission exceeding the absorption which in turn is a consequence of the "inverted population" of the statistical states. Hence, these instabilities may be appropriately called the "causal or kinetic" instabilities. That is, the growth rates of these instabilities are a consequence of the causality principle and thus depend on the difference in population of states or on the slope of the particle distribution function. In other words, the growth rates of these instabilities are determined by the imaginary or the anti-Hermitian part of the causal dielectric tensor appropriate to the system under study. However, it should be emphasized that in contrast to the conventional inverse Landau damping which cepends on the slope of the particle distribution function in the direction of its wave vector $k$ at and around its phase velocity $v_{p}=\omega / k$, the inverse cyclotron damping under study does not deperid on the slope of the perpendicular distribution function $f_{\perp}\left(v_{\perp}\right)$ as can be seen by integrating by parts the expression for $\gamma$, but depends sensitively on the slope of only the parallel distribution function at and around the cyclotron phase velocity $v_{p} i_{z}=\left[\left(\omega-m \omega_{c i}\right) / k_{\|}\right] i_{z}$ via the conventional cyclotron overstability terms of the standard hot plasma theory. The physical reason for this, as seen from Eqs. (3) and (4), is that the particle-wave resonance condition is only on the parallel distribution function and is totally independent of the nature of the perpendicular distribution function. This again is a consequence of the fact that the Doppler shift is determined by the conservation of total energy and the conservation of only the parallel canonical momentum for the cyclotron emission and absorption processes under consideration.

These causal or kinetic instabilities can of course be convective or absolute in nature. ${ }^{54}$ In the formalism outlined here we have used the temporal photon master equation to describe the growth or damping in time of the electromagnetic waves everywhere in the plasma volume under 
consideration. Hence we are in principle assuming that the instability is absolute in nature, i.e., a growing disturbance that does not propagate. However, convective instabilities describing growing disturbances that propagate can also be analyzed using the spatial master equations of nonequilibrium statistical mechanics. In contrast to these causal or kinetic instabilities, which are determined by the anti-Hermitian part $K_{a}=(2 i)^{-1}\left[K-K^{+}\right]$of the system's dielectric tensor $K$, one can also have hydrodynamic instabilities which are determined only by the Hermitian part $\mathbf{K}_{\mathrm{h}}=(1 / 2)\left[\mathbf{K}+\mathbf{K}^{+}\right]$and the noncausal plasma dispersion relation

$$
\mu \times(\mu \times \mathbf{E})+\mathbf{K}_{\mathbf{h}} \cdot \mathbf{E}=0,
$$

where $\mu=c k / \omega$ is the vector index. of refraction of the system under study. These hydrodynamic instabilities can be obtained by setting the secular determinant of Eq. (78) equal to zero. In the cold plasma theory $K_{h}$ is the cold plasma dielectric tensor as found in Stix, ${ }^{24}$ for example. But in general, $\mathbf{K}_{\mathbf{h}}$ is the Hermitian part of the hot plasma dielectric tensor which contains all the finite Larmor radius effects. The Hermitian part $\mathbf{K}_{h}$ and the anti-Hermitian part $\mathbf{K}_{\mathbf{a}}$ are, of course, related by the well-known Kramers-Kronig relations so as to satisfy the laws of causality. In solving the noncausal dispersion relation of the secular determinant equal to zero of Eq. (78), we can either assume a real $k$ and look for complex $\omega$ or assume a real $\omega$ and solve for the complex $k$. These solutions in general will give either growing or damped waves and such growing sclutions are the ones that give rise to these hydrodynamic instabilities. These hydrodynamic instabilities depend only on the Hermitian part, $\mathbf{K}_{h}$, while the causal or kinetic instabilities depend only on the anti-Hermitian part, $\mathbf{K}_{\mathbf{a}}$, of the system's dielectric tensor $\mathbf{K}$. That is, these instabilities can be understood in terms of the hydrodynamic conservation law equations for the mass, momentum, energy density, etc., which are obtained by taking the various velocity moments of the equations of the kinetic theory, and are, hence, independent of the detailed nature such as the shapes and slopes of the particle distribution functions. Here again, these hydrodynamic instabilities can, of course, be either convective (i.e., a growing disturbance that propagates) or absolute (a growing disturbance that does not propagate) in nature. The well-known example of the distinction between the kinetic and hydrodynamic instability is in the nature of the familiar beam-plasma two stream instability. $55,56,45$ If the beam is a "gentle bump," then the instability is causal or kinetic and the growth rate is determined by the conventional inverse Landau damping 55 which is linear in the ratio of the beam density to the plasma density $\left(n_{b} / n_{e}\right)$. However, if the beam is monoenergetic and is not a "gentle bump," then the instability is hydrodynamic in nature 56 and the growth rate is proportional to $\left(n_{b} / n_{e}\right)^{1 / 3}$ and is not detęrmined by the familiar inverse Landau damping. It is shown in Ref. 56 that the hydrodynamic instability resulting from the interaction of a plasma with a monoenergetic beam in the course of time evolves into a kinetic or causal instability which then 
evolves towands the quasilineat ind/or the nonlinear steady state. That is, the feedback effect of the unstable oscillations retards the beam and increases its temperature and thus the initially monoenergetic beam evolves into a "gentle bump." The initial evolution of the monoenergetic beam-plasma system towards a gentle bump condition is given by a hydrodynamic description while the final evolution from this gentle bump condition towards the quasilinear steady state is given by the kinetic description. Thus the eventual steady state is the kinetic quasilinear and/or nonlinear steady state regardless of whether the initial instability is "hydrodynamic" or "kinetic or causal" in nature. Here we have only examined the causal or kinetic instabilities which are driven by the inverse cyclotron damping in $\mathbf{K}_{\mathrm{a}}$. In a subsequent paper we will try to examine the consequences of the hvdrodynamic instabilities of Eq. (78) due to $\mathbf{K}_{\mathbf{h}}$ in the neighborhood of ion cyclotron harmonics.

\section{SOME NUMERICAL ESTMMATES FOR TYPICAL TOKAMAK PARAMETERS}

We now wish to make some approximate numerical estimates of the power emission both by the fusion product protons and alpha particles, and by the hackground deruterium plasmas ions in their ion cyclotron range of frequencies and at the proton spin-tlip nsonance frequency for typical tokamak parameters such as those of TFTR and JET. In all the experiments done on 'TFTR, the plasma was dominated by the DD fusion reactions of Eq. (1). This is also true for the oldar JET experiments. However, in the recent JET experiments, the plasna was dominated by the DT fusion reactions of Eq. (2a), i.e., these discharges had a plasma composition ratio (T to $D+T$ density) of about 11\%. It is shown elsewhere 57 that for the present plasma conditions in TFTR and JET, the burnup fraction of $1 \mathrm{MeV}$ tritons is approximately 1\%. This implies that in DD fusion reaction dominated plasmas, the fusion product protons and tritons are of equal number density $\left(n_{p}=n_{t}\right)$, and the alpha particle density $n_{\alpha}=10^{-2} n_{p}$. Further, since the two primary reactions of Eq. (1) occur with equal probability, in these DD reaction dominated plasmas there will be an equal number of ${ }^{3}$ He particles. We will see later that it is only the protons and alpha particles that have velocities larger than the Alfven speed and, consequently, are capable of emitting equally intense barmonics at their corresponding cyclotron frequencies. Hence, for these DD fusion reaction dominated plasmas, the contribution to $\mathrm{PICE}_{\mathrm{I}}$ is mainly from the protons (at n $\omega_{\mathrm{Cp}}$ ) with a $1 \%$ contribution from the alpha particles (at $m \omega_{c \alpha}=m \omega_{c d}$ ). Since the ratio of the crosssections 58 for the reaction DT to those of the reaction DD is approximately equal to $10^{2}$, in DT fusion reaction dominated plasmas the main contribution to PICE is from the alpha particles (at $\left.m \omega_{c \alpha}=m \omega_{c d}\right)$ with about $1 \%$ contribution from the protons. That is, the ratio [PICE from alpha particles / PICE from protons] is about $10^{-2}$ for DD fusion reaction dominated plasmas (TFTR and 
the older JET data) and is about $10^{2}$ for the DT fusion reaction dominated plasmas (recent JET results). Further, it is apparent that if the observed broader background continuum component of the spectrum (of Fig. 1) is due to the proton spin-flip emission in the DD fusion reaction dominated plasmas, then such emission should be down by a factor of about $10^{4}$ in the corresponding DT fusion reaction dominated plasmas. That is, the ratio $\left[\mathrm{PBBCC}_{\mathrm{BB}} \mathrm{PICE}\right]$ for the $\mathrm{DT}$ fusion reaction dominated plasmas is about $10^{-4} \times\left[\mathrm{P}_{\mathrm{BBCC}} / \mathrm{P}_{\mathrm{ICE}}\right]$ of the corresponding $\mathrm{DD}$ fusion reaction dominated plasmas, where $\mathrm{P}_{\mathrm{BBCC}}$ is the total integrated power in the broader background continuum component of the emission spectrum. With this in mind, we will present our PICE estimates only for the fusion product protons since similar estimates apply equally well for the alpha particles, and the correct combination can easily be worked out for the DD and the DT fusion reaction dominated plasmas.

\section{A. Chosen typical plasma parameters and relevant geometrical considerations}

We take the following tokamak plasma parameter conditions: The background plasma is of deuterium ions with $n_{e}=n_{i}=n_{d}=5 \times 10^{13} \mathrm{~cm}^{-3}$ and $T_{\perp e}=T_{\| l}=T_{\perp i}=T_{\| i}=T=5 \mathrm{keV}$, the direc: $d$ energy of the newly born fusion protons $E_{p}=\left(M_{p} V_{p}^{2} / 2\right)=3 \mathrm{MeV}$ and the amount of thermal spread $K T_{p}$ in the fusion proton directed energy $E_{p}$ according to Brysk ${ }^{59}$ is $\left({ }^{\prime \prime 2} T_{p}\right)=\left(T_{i} E_{p}\right)^{1 / 2}=120 \mathrm{keV}$ for the background deuterium ion temperature $T_{i}=5 \mathrm{keV}$, the tokamaks major radius $R_{0}=2.65 \mathrm{~m}$, the plasma major radius $R_{p}=2.45 \mathrm{~m}$, the plasma minor radius $a_{p}=80 \mathrm{~cm}$, the minor radius of the tokamak's vacuum vessel af $=1.2 \mathrm{~m}$, the major radius of the resoriant iyclotron layer is $R_{c}=\left(R_{p}+a_{p}\right)=3.25 \mathrm{~m}$, the confining magnetic field $B=4.45 \mathrm{~T}=4.45 \times 1)^{4}$ Gauss at $R=R_{0}$, and the fraction of the newly born proton population is $\eta=\left(n_{p} / n_{i}\right)=\left[n_{p} /\left(n_{d}+n_{p}\right)\right]$. The scrape-off plasma layer radius $a_{s c}=\left(a_{p}+1.5\right) \mathrm{cm}=95 \mathrm{~cm}$, i.e., the scrape-off layer is from $a_{p}$ to $a_{s c}$. At the plasma radius $a_{p}$, $n_{e}=n_{i}=n_{d}=1.0 \times 10^{12} \mathrm{~cm}^{-3}$, and $T_{e}=T_{i}=100 \mathrm{eV}$. In the scrape-off plasma the density and temperature profiles are exponential with an e-folding length of about $2.5 \mathrm{~cm}$. In general, both in TFIR and JET, the experimentally observed cyclotron resonance layer $R_{c}$ is located in the scrapeoff layer plasma, i.e., $\left(R_{p}+a_{p}\right) \leq R_{c}<\left(R_{p}+a_{s c}\right)$. We will also examine the situation of the injection of a neutral beam of hydrogen of energy $E_{b}=100 \mathrm{keV}$ into this background deuterium plasma.

Thus at the plasma center $\left(R_{p}\right)$, the electron cyclotron frequency $\left(\omega_{c e} / 2 \pi\right) \approx 1.35 \times 10^{11} \mathrm{~Hz}$, the proton cyclotron frequency $\left(\omega_{c p} / 2 \pi\right)=73.34 \mathrm{MHz}$, the deuteron cyclotron frequency $\left(\omega_{\mathrm{cd}} / 2 \pi\right) \approx 36.67 \mathrm{MHz}$, the proton spm-flip resonant frequency $\left(\omega_{\mathrm{sp}} / 2 \pi\right)=\left(\mathrm{g}_{\mathrm{p}} \omega_{\mathrm{cp}} / 2\right) \approx$ $5.59 \times 36.67 \mathrm{MHz}=205 \mathrm{MHz}$. At the outer low-field side plama edge $\left(R_{c}=R_{p}+a_{p}\right)$, $\left(\omega_{c e} / 2 \pi\right) \approx 1.02 \times 10^{11} \mathrm{~Hz},\left(\omega_{c p} / 2 \pi\right) \approx 55.29 \mathrm{MHz},\left(\omega_{c d} / 2 \pi\right) \approx 27.64 \mathrm{MHz}$, and 
$\left(\omega_{\mathrm{sp}} / 2 \pi\right) \approx 154.5 \mathrm{MHz}$. The plasma dielectric coefficient for Alfven waves $C=K_{h}=\mu^{2} \approx\left(4 \pi n_{d} M_{d} c^{2} / B^{2}\right) \approx 815.7$, i.c., the Alfven wave index of refraction $\mu \approx 28.56$ and hence the Alfven wave phase velocity $V_{A}=(\omega / k) \approx 1.05 \times 10^{9} \mathrm{~cm} / \mathrm{sec}$. It should be noted that the "dressing" factor $19,20 \mathrm{C}=\mu^{2}$ comes from the eigenmode Fourier analysis of the electromagnetic (wave) field energy inside the whole tokamak plasma, i.e., the box of volume $\mathrm{L}^{3}$ under consideration in Eqs. (3), (4), and (21). Hence, the Alfven wave index of refraction $\mu$ and the corresponding Alfven wave phase velocity $\mathrm{V}_{\mathrm{A}}$ are to be taken as average values for the entire plasma column, regardless of the location of the resonant emitting layer. That is, even if the resonant layer is in the scrape-off region of the plasma where the ion density $\mathrm{n}_{\mathrm{d}}$ is very very low, we must use this average value of $\mu=28.56$, and not the locally evaluated value of $\mu \propto\left(n_{d}^{1 / 2} / B\right)$, for our emission calculations everywhere. Here, we are using a particle-orbit analysis, and the global features of the background medium enter via this "dressing" factor $C$. In the usual kinetic dispersion theory analysis, the Alfven wave dispersion $\omega=k V_{A}$ comes naturally as an average global quantity for the eigenmodes of the plasma column. The velocity of the $3 \mathrm{MeV}$ protons is $V_{p}=2.39 \times 10^{9} \mathrm{~cm} / \mathrm{sec}$, i.e., $\left(\mathrm{V}_{\mathrm{p}} / \mathrm{V}_{\mathrm{A}}\right)=2.28$, the velocity of the $3.6 \mathrm{MeV}$ alpha particle is $V_{\alpha}=1.31 \times 10^{9} \mathrm{~cm} / \mathrm{sec}$, i.e., $\left(V_{\alpha} / V_{A}\right)=1.25$, the velocity of $T_{i}=5 \mathrm{keV}$ deuterium plasma ions is $v_{d}=6.9 \times 10^{7} \mathrm{~cm} / \mathrm{sec}$, i.e., $\left(v_{d} / V_{A}\right)=0.0656$, the thermal spread speed of protons $v_{p}=\left(2 K T_{p} / M_{p}\right)^{1 / 2}=3.38 \times 10^{8} \mathrm{~cm} / \mathrm{sec}$ for $T_{p}=60 \mathrm{keV}$, i.e., $\left(v_{p} / V_{A}\right)=0.322$. The electron plasma frequency $\left(\omega_{\mathrm{p}} / 2 \pi\right) \approx 6.35 \times 10^{10} \mathrm{~Hz}$ for $\mathrm{n}_{e} \approx 5 \times 10^{13} \mathrm{~cm}^{-3}$, the deuterium ion plasma frequency $\left(\omega_{\mathrm{pd}} / 2 \pi\right)=1.048 \times 10^{9} \mathrm{~Hz}$, the proton ion plasma frequency $\left(\omega_{\mathrm{pp}} / 2 \pi\right) \approx 1.482$ $\times 10^{9} \times \eta^{1 / 2} \mathrm{~Hz}$, and we will make use of the relations $\left(M_{d} / 2\right)=M_{p}=1837.05 M_{c}$ and $\left(\mathrm{M}_{\mathrm{e}} \mathrm{C}^{2}\right)=0.511 \mathrm{MeV}$. The velocity of the neutral hydrogen beam at energy $\mathrm{E}_{b} \approx 100 \mathrm{keV}$ is $\mathrm{V}_{\mathrm{b}} \approx$ $4.364 \times 10^{8} \mathrm{~cm} / \mathrm{sec}$, i.e., $\left(\mathrm{V}_{\mathrm{b}} / \mathrm{V}_{\mathrm{A}}\right)=0.42$.

If $\Delta \omega$ is the full width at half maximum of the cyclotron emission lines, then the width in major radius of this emitting resonant layer is $\Delta R_{c} \approx R_{c}(\Delta \omega / \omega)$ since the cyclotron frequency $\omega_{c i} \propto B \propto R^{-1}$. The chordal vertical height of this resonant layer plasma $=2\left[a_{s c}^{2}-\left(R_{c}-R_{p}\right)^{2}\right]^{1 / 2}=2\left[a_{s c}^{2}-a_{p}^{2}\right]$, and the total circular length of this resonant layer - $2 \pi R_{c}$. Hence the emitting plasma volume contained in this resonant layer $\left(\Delta V_{0}.\right)=\left(2 \pi R_{c}\right)\left(\Delta R_{c}\right)\left(2\left[a_{s c}^{2}-a_{p}^{2}\right]^{1 / 2}\right\}=4 \pi R_{c}^{2}\left[a_{s c}^{2}-a_{p}^{2}\right]^{1 / 2}(\Delta \omega / \omega)=6.80 \times 10^{7} \times(\Delta \omega / \omega)$ $\mathrm{cm}^{3}$. The internal surface area of the tokamak's vacuum vessel $S=\left(2 \pi \mathrm{R}_{0}\right)\left(2 \pi \mathrm{a}_{0}\right)=1.26 \times 106$ $\mathrm{cm}^{2}$, and we will take the collecting area $(\Delta S)$ of the receiving antenna as $(\Delta S)=10 \mathrm{~cm}^{2}$. This value of $(\Delta S)$ seems reasonable for the TFTR data which was taken with small radio-frequency probes. However, the JET results yielding $\mathrm{P}_{\mathrm{ICE}}$ in the range $10^{-12} \leq \mathrm{P}_{\mathrm{ICE}} \leq 10^{-5} \mathrm{~W}$ reported in Refs. 3 and 4 was taken with the large ICRF heating antennas as the ICE receiving probes and hence for these JET data $(\Delta S)$ is probably a factor of $10^{3}$ or so higher, i.e., $(\Delta S)=10^{4} \mathrm{~cm}^{2}$. If 
$P(m)$ is the emitted power per unit volume from this resonant layer in the $m$-th harmonic, then the total power $\mathrm{P}_{\mathrm{A}}(\mathrm{m})$ collected by the receiving antenna for $\omega \approx m \omega_{c i}$ is $\mathrm{P}_{\mathrm{A}}(\mathrm{m}) \approx \mathrm{P}(\mathrm{m})(\Delta \mathrm{Vol}$.) $(\Delta S / S) \approx P(m) \times 5.40 \times 10^{2} \times(\Delta \omega / \omega)$ for TFTR and $P_{A}(m) \approx P(m) \times 5.40 \times 10^{5}(\Delta \omega / \omega)$ for JET. This analysis applies only for the volume emission processes such as the spontaneous emission when the optical depth $\tau \ll 1$. Further, since the free-space wave length of the radiation is large compared to the plasma diameter, we have assumed that the emitted radiation will fall evenly over the entire inner vacuum vessel surface.

If the optical depth $\tau \gg>1$ and if the system is in thermodynamic equilibrium, it is no longer a volume emission and the system emits as a black-body from the front surface of the resonant (black-boily) layer $\mathbf{R}_{c}$. Then the power received by the antenna 4 is $P_{A}(m)=\left[k T_{r}\left(\omega=m \omega_{c i}\right)\right]\left[1-p^{2}\right](\Delta f)(\Delta \Omega)(\Delta S)$, where $p$ is the voltage reflection coefficient at the antenna-plasma interface and $(\Delta \mathrm{P})(\Delta \Omega)$, and $(\Delta S)$ are the receiver bandwidth, antenna solid angle, and the antenna area, respectively. Here $T_{r}\left(\omega=m \omega_{c i}\right)$ is the effective radiation temperature, and as derived in Eq. (56) it is equal to $T_{\perp i}$ at complete thermodynamic equilibrium. This effective radiation temperature formula for $\mathrm{P}_{\mathrm{A}}(\mathrm{m})$ is also applicable to nonequilibrium systems as long as the emission under study is a surface emission and the optical depth $\tau$ remains very much larger than unity. However, if the system under study behaves as a laser or a maser it is not clear how the total power received by the antenna $P_{A}(m)$ is related to the total laser power $P_{L}(m)$ emitted by the system. Of course, one can always say that $P_{A}(m)$ has to be less than or equal to $P_{L}(m)$.

\section{B. Estimates of single dressed test particle spontaneous emission}

Let us first examine the predictions of our calculations of spontaneous emission. We must, of course, assume that $\tau \ll 1$ and this is a volume emission. According to our Eq. (10), for example, we find that the spontaneous emission from the fusion protons $P(1)=\eta \times 4.98 \times 10^{-8} \mathrm{~W} \mathrm{~cm}^{-3}$ for the region near $R_{c} \approx R_{p}+a_{p}$, or equivalently, $1.66 \times 10^{-20} \mathrm{~W}$ per proton. Since the emission is proportional to $\left\langle v_{\perp}^{2}\right\rangle$, we note that the equivalent alpha particle emission $\mathrm{P}_{\alpha}(\mathrm{m})$ can be obtained by multiplying the corresponding proton emission $P_{p}(m)$ by the factor $\left[(3.6 \mathrm{MeV} / 3 \mathrm{MeV})\left(\mathrm{M}_{\mathrm{p}} / \mathrm{M}_{\alpha}\right)\right]=0.3$. Here, $\theta=0, k=k_{\|} i_{z}$, $\omega \approx k V_{A}=k_{\|} V_{A}$, and the Doppler broadening $(\Delta \omega) \approx 2 k_{\|}\left(2 k T_{p} / M_{p}\right)^{1 / 2}$. That is, $(\Delta \omega / \omega)=2\left(2 \times T_{p} / M_{p} V_{A}^{2}\right)^{1 / 2} \approx 0.645$. Hence $P_{A}(1)=5.40 \times 10^{5} \times \eta \times 4.98 \times 10^{-8} \times 0.645 \mathrm{~W}$ $\approx \eta \times 1.73 \times 10^{-2} \mathrm{~W}$, and $P_{A}(m \geq 2)=0$ for JET and is a factor of $10^{3}$ lower for TFTR. Thus, for example, when the fractional proton (and/or alpha particle) population $\eta=10^{-4}$, then $P_{A}(1)=1.73 \times 10^{-6} \mathrm{~W}$. This amount of power is in reasonable agreement with those experimentally observed ${ }^{4}$ in JET but the theoretically expected Doppler width $(\Delta \omega / \omega)$ is larger than the experimentally observed line widths. Further, at $\theta=0, k_{\perp}=0$, and, hence, we can only 
account for the emission at the fundamental proton (and/or alpha particle) cyclotron frequency. There is no emission at higher harmonics without a finite value of $\mathrm{k}_{\perp}$. It should be noted that the result of Eq. (10) is exactly the classical electrodynamics formula for the total emission from an accelerated charge in a medium of index of refraction $\mu$ as $\left(\mu 2 \mathrm{q}_{1}^{2} \mathrm{a}^{2} / 3 \mathrm{c}^{3}\right)$, where for the circular cyclotron motion the acceleration $a=\left(v_{\perp}^{2} / \rho_{L}\right)=\left(v_{\perp} / \rho_{L}\right)\left(\rho_{L} \omega_{c i}\right)=\left(\omega_{c i} v_{\perp}\right)$. For finite $k_{\perp}=k \sin \theta$, it is seen from Eq. (12) that

$$
\frac{P(m+1)}{P(m)} \approx(m+1)^{-2}(m \sin \theta)^{2}\left[\left\langle\left(\frac{v_{1}}{V_{A}}\right)^{2}\right\rangle\right],
$$

where for $3 \mathrm{MeV}$ protons $\left[\left\langle\left(v_{\perp} N_{A}\right)^{2}\right\rangle\right]=\left(V_{p} / V_{A}\right)^{2}=(2.28)^{2}=5.20$. Thus from Eq. (79) we see that the approximate angle $\theta_{m}$ at which $P(m+1)=P(m)$ is given by

$$
\sin \theta_{\mathrm{m}} \approx \frac{\mathrm{m}+1}{2.28 \mathrm{~m}}
$$

Thus for $\mathrm{m}=1,2$, and $3, \theta_{\mathrm{m}}$ takes the approximate values $61.30^{\circ}, 41.15^{\circ}$, and $35.80^{\circ}$, respectively. A more rigorous estimate of these angles can be obtained from the results found in Eqs. (16) and (17). For these angles the second, third, and the fourth harmonics, respectively, are as intense as the fundamental. Indeed, it is seen from Eq. (79) that if $\left[\left\langle\left(v_{\perp} / N_{A}\right)^{2}\right\rangle\right]^{1 / 2}$ is larger than $[1+(1 / m)]$, there always exists a $\theta_{m}$ between zero and $\pi / 2$ for which the $(m+1)$-th harmonic will have the same emission intensity as that of the $m$-th harmonic. Thus we see that the spontaneous emission from the fusion product protons can account for all the cyclotron harmonic emission at frequencies $\omega \approx m \omega_{c p}$, where $1 \leq \mathrm{m} \leq \mathrm{m}_{0}$ such that mo $\omega_{c p}$ is less than the lower hybrid frequency $\omega_{L H}$. These conclusions can be verified and substantiated by the explicit results of Eqs. (13) to (17) and the plots of Fig.2.

Let us now use Eqs. (10) and (12) to estimate the spontaneous emission from the deuterium ions of the background plasma. From Eq. (10) we obtain that $P(1)=3.46 \times 10^{-10} \mathrm{~W} \mathrm{~cm}^{-3}$ and $(\Delta \omega / \omega)=2\left(v_{d} / V_{A}\right)=0.131$ for the background deuterium ions. Hence the total power collected by the receiving antenna at the fundamental deuterium cyclotron frequency $\omega_{c d}=\left(\omega_{c p} / 2\right)$ is $\mathrm{P}_{\mathrm{A}}(1)=5.40 \times 10^{-5} \times 3.46 \times 10^{-10} \times 0.131 \mathrm{~W}=2.45 \times 10^{-5} \mathrm{~W}$ and $\mathrm{P}_{\mathrm{A}}(\mathrm{m} \geq 2)=0$ for JET and is a factor of $10^{3}$ lower for TFTR. This amount of power is in reasonable agreement with that experimentally observed 4 in JET. However, for these deuterium ions of the background plasma $\left[<\left(v_{\perp} / v_{A}\right)^{2}>\right]^{1 / 2} \approx 0.0656$. Hence from Eq. $(79),[P(m+1) / P(m)] \approx(m+1)^{-2}(m \sin \theta)^{2}$ $(0.0656)^{2} \approx[\mathrm{m} \sin \theta /(\mathrm{m}+1)]^{2} \times 4.30 \times 10^{-3}<4.30 \times 10^{-3}$. Thus the intensity of successive harmonics of the deterium cyclotron emission should decrease by at least a factor of hundred or 
more. These conclusions can be verified and substantiated by the explicit results of Eqs. (12), (14), (17), (36), and (37). Thus the experimentally observed emission at $\omega \approx \omega_{\text {cd }}$ can be accounted for by the direct spontaneous emission by the deuterium ions of the background plasma. However, the emission at the second and higher harmonics of $\omega_{c d}$ cannot be accounted for by such direct spontaneous emission by the deuterium ions.

We now wish to make an estimate of the direct spontaneous spin-flip emission from these fusion product protons. For this purpose we will make use of Eq. (27), and since $\left\langle G_{1}\right\rangle=(1 / 4)\left\langle\left(v_{\perp} / V_{A}\right)^{2}\right\rangle=(1 / 4)(2.28)^{2}=1.30$, we find from Eq. (27) that $\left[P_{S}(+1) / P(1)\right] \approx 4.37 \times 10^{-24}$. That is, the direct spontaneous spin-flip emission by the fusion protons is a factor of at least $10^{-23}$ lower than the corresponding direct spontaneous fundamental cyclotron emission by these protons. Hence the direct spontaneous spin-flip emission from the fusion product protons cannot account for the experimentally observed broader background continuum component of the spectrum in TFTR, despite the fact that this continuum component exists roughly over the frequency range for which the proton spin-flip resonance is within the plasma, see Fig. 1.

\section{Estimates of black-body emission}

We will now turn our attention to numerical estimates of our theoretical formulae of Sec. III on the linearized theory of the radiative steady state. At thermodynamic equilibrium, this radiative steady state is the state of radiative equilibrium. That is, the radiation field inside the medium (i.e., the field oscillators) is in complete thermodynamic equilibrium with the radiating particles (i.e., the particle oscillators). At this steady state the classical equipartition theorem should be valid, i.e., the energy per degree of freedom should be equal to $\mathrm{kT} / 2$. Since the emission and absorption of these cyclotron harmonic waves is due to the finite size of the ion Larmor orbits, it is the perpendicular motion of the ions that is in radiative equilibrium with its own radiation field. This perpendicular ion motion has two degrees of freedom, one corresponding to motion along the $x$-axis and the other to the motion along the $y$-axis. The energy of these two degrees of freedom is $2\left(K T_{\perp i} / 2\right)=\kappa T_{\perp i}$. Hence according to the classical equipartition theorem the effective radiation temperature $T_{r}$ must equal $T_{\perp i}$ at this radiative equilibrium, i.e., the wave energy density $\varepsilon=\kappa T_{r}$ is in equipartition with the ion perpendicular energy density $k T_{L i}$. Similarly, at radiative equilibrium for spin-flip emission and absorption, the effective radiation temperature $T_{r}$ should be equal to its effective spin temperature $T_{S}$. These are the results found in our Eqs. (56) and (59), respectively. Also in Eqs. (49) and (52), we may write $\left(S_{m}^{-1} \partial\left\{\left[S_{m} J_{m}^{\prime}\right]^{2}\right\} / \partial S_{m}\right)=$ $\left(2 \partial\left[\left[S_{m} J_{m}^{\prime}\right]^{2}\right\} / \partial S_{m}^{2}\right) \approx\left\{\left(2 / S_{m}^{2}\right)\left[S_{m} J_{m}^{\prime}\right]^{2}\right\}$ and $\left(S_{m}^{-1} \partial\left\{\left[S_{m} J_{m}\right]^{2} / 2\right\} / \partial S_{m}\right) \approx\left\{\left(2 / S_{m}^{2}\right)\left[S_{m} J_{m}\right]^{2} / 2\right\}$, then the results of these Eqs. (49) and (52) both reduce to yield $\kappa T_{r} \approx\left(M_{i} V_{A}^{2} / m^{2}\right)\left(S_{m}^{2} / 2\right)=$ 
$\left(M_{i} V_{A}^{2} / m^{2}\right)\left(m^{2} V_{i}^{2} / 2 V_{A}^{2}\right)=\left(M_{i} V_{i}^{2} / 2\right)$, i.e., the radiation field energy is in equipartition with the particle kinetic energy.

If the system is in radiative equilibrium then the power it will emit into a receiving antenna with a bandwidth $\Delta f$ is $P_{A} \approx\left[\kappa T_{r} \Delta f\right]=\left[\left(\kappa T_{r} m \omega_{c i} / 2 \pi\right)(\Delta \omega / \omega)\right] \approx\left[1.602 \times 10^{-19} \times\left(m \omega_{c i} / 2 \pi\right)\right.$ $(\Delta \omega / \omega)] \mathrm{W}$ per $\mathrm{eV}$, where we have used the fact that $\omega \approx m \omega_{c i}$, and $\mathrm{m}$ is the harmonic number. Thus the black-body emission from the $3 \mathrm{MeV}$ protons with $\left(\omega_{\mathrm{ci}} / 2 \pi\right) \approx 55.29 \mathrm{MHz}$ is $P_{A} \approx\left[2.66 \times 10^{-5} \mathrm{~m}(\Delta \omega / \omega)\right] \mathrm{W}$. If we now take the experimental value of $(\Delta \omega / \omega) \approx 0.05$, then $P_{A} \approx\left[1.33 \times 10^{-6} \mathrm{~m}\right] \mathrm{W}$. This emission increases lineariy with the harmonic number $\mathrm{m}$ if $(\Delta \omega / \omega)$ is a constant. This is a huge amount of power compared to the early experimentally observed values ${ }^{4}$ from JET in the range $10^{-12} \leq P_{I C E} \leq 10^{-9} \mathrm{~W}$. But recent JET measurements reported by Sadler 4 gives this new range as $10^{-11} \leq \mathrm{P}_{\mathrm{ICE}} \leq 10^{-5} \mathrm{~W}$. However, for these recent measurements the total neutron source rate is also accordingly much higher. That is, $10^{12} \leq$ total neutron source rate $\leq 10^{18} \mathrm{sec}^{-1}$ for the recent JET measurements compared to their old range of $10^{10} \leq$ total neutron source rate $\leq 10^{13} \mathrm{sec}^{-1}$. Thus it appears that in the JET experiments the emission from the fusion product protons and alpha particles is a volume emission due to the single particle spontaneous emission which is proportional to the fusion product number density, and is not a collective black-body surface emission from the antenna viewing face of the resonant layer which only depends on the surface temperature and is totally independent of the fusion product number density. We wish to point out that although the JET results show a remarkable linear correlation between the measured second harmonic ICE peak power and the measured total neutron flux over six orders in magnitude, the TFTR results do not show such a clear cut correlation between the amplitude of the $\mathrm{n}=2 \mathrm{ICE}$ peak and the fusion product number density. However, for black-body emission from the $5 \mathrm{keV}$ deuterium ions of the background plasma we find that $\mathrm{P}_{\mathrm{A}}=\left[2.21 \times 10^{-8} \mathrm{~m}(\Delta \omega / \omega)\right] \mathrm{W}$ with $\left(\omega_{\mathrm{ci}} / 2 \pi\right) \approx 27.64 \mathrm{MHz}$. Thus on making use of the experimental value of $(\Delta \omega / \omega)=0.05$, we get $P_{A}=\left[1.105 \times 10^{-9} \mathrm{~m}\right] \mathrm{W}$, a value that is similar to the experimental observations. Now in the case of fusion product proton spin-flip emission, the spin temperature $T_{S}$ is most likely to be equal to its translational temperature $T_{V}$. Only in solid state physics $T_{S}$ can differ significantly from $T_{V}$. In general, in any decay processes, the daughter products has to inherit at least the thermal spread of their parent particles owing to the intrinsic conservation laws of both the energy and momentum in the microscopic level. However, Brysk 59 has shown that the mean thermal spread $k T_{p}$ in the fusion proton directed energy $E_{p} \approx 3 \mathrm{MeV}$ is given by $\left(2 T_{p}\right) \approx\left[4 M_{p} T_{i} E_{p} /\left(M_{p}+M_{T}\right)\right]^{1 / 2} \approx\left(T_{i} E_{p}\right)^{1 / 2} \approx 120 \mathrm{keV}$ for the reaction of Eq. (1), where $T_{i}$ is the temperature of the background deuterium plasma ions and $M_{p}$ and $M_{T} \approx 3 M_{p}$ are the masses of the proton and the triton, respectively. Hence, it follows from Eqs. (1) and (2) that the fusion product protons will carry the thermal spread $T_{p}$ of about $60 \mathrm{keV}$ (from the background 
deuterium plasma ions of $T_{i}=5 \mathrm{keV}$ ) superimposed on their directed energy of $3 \mathrm{MeV}$. That is, $T_{S} \approx T_{v} \approx T_{p} \approx 60 \mathrm{keV}$. Thus the black-body proton spin-flip emission will be almost $(60 / 5)=12$ times larger than the black-body cyclotron emission from the background deuterium plasma ions. Here from Eq. (18), $\omega_{s p}=g_{p} \omega_{c d}$, and experimentally one finds that for the background continuum spectrum $(\Delta \omega / \omega) \approx 1$, we obtain the black-body spin-flip emission power as $\mathrm{P}_{\mathrm{A}} \approx 1.48 \times 10^{-6} \mathrm{~W}$. By using a triangular approximation to the broader background continuum component of the TFTR spectrum of Fig. 1, we find that the total integrated power in this continuum component is $\mathrm{PBBCC}_{\mathrm{BB}} \approx\left[\left(2 \times 10^{-10} \mathrm{~W}\right)(600 \mathrm{MHz} / 2)\right] /(300 \mathrm{kHz})=2 \times 10^{-7} \mathrm{~W}$, where we have used the fact that the bandwidth $\Delta f$ of the TFTR receiver is $300 \mathrm{kHz}$. Thus we see that the black-body spin-flip emission power is somewhat larger than what is observed experimentally.

\section{Estimates of optical depths}

It is now of interest to us to carefully examine whether the systems studied above can indeed reach the state of radiative equilibrium. In general, any system can reach a radiative steady state if and only if the self- absorption of these radiations by the system is almost totally complete. That is, the optical depth $\tau$ of the system for these radiation fields must be very much larger than unity. Hence we now wish to make numerical estimates of $\tau$ of Eqs. (48), (51), (55), and (58), i.e., we need to know the values of $\tau$ for cyclotron harmonic emission both from the background deuterium ions and from the fusion product protons and also $\tau$ for the proton spin-flip emission. Since $\tau$ will take its largest value near the plasma center, we will only examine this case of $R_{c}=R_{s p} \approx R_{p}$. On making use of the parameter numbers presented earlier we find that $Y_{d}=Y=\left(\pi R_{c} \omega_{p i}^{2} / 2 \mu^{2} \omega_{c i} V_{A}\right)$ $\approx\left[1.121 \times 10^{2}\right]$ for the deuterium background plasma, and $Y_{p}=\eta Y=\left[1.121 \times 10^{2} \times \eta\right]$ for the fusion product protons. For $s_{m}^{2} \ll 1$, Eq. (36) yield $\left[\left\langle G_{m}>\right] \approx m^{2} I_{m}\left(s_{m}^{2} / 2\right)\left[1+\left(V_{z} / V_{A}\right)^{2}\right] \approx\right.$

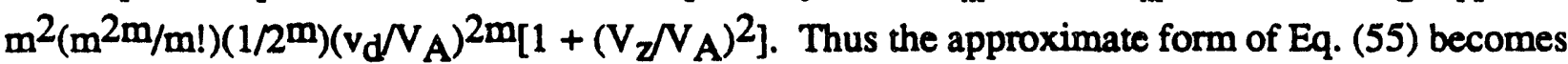
$\tau \approx\left(Y_{d} / m\right)\left(2 / m^{2}\right)\left(V_{A} / v_{d}\right)^{2}\left[<G_{m}>\right] \approx\left\{Y_{d}\left[m(2 m-1) / m ! 2^{(m-1)}\right]\left[\left(v_{d} / V_{A}\right)^{(2 m-2)}\right]\left[1+\left(V_{z} / V_{A}\right)^{2}\right]\right\}$. Hence assuming $V_{z} \ll V_{A}$ for $m=1, \tau \approx Y_{d} \approx 1.121 \times 10^{2}$; for $m=2, \tau \approx 2 Y_{d}(0.0656)^{2} \approx$ 0.965 ; and for $m=3, \tau \approx 3 Y_{d}(3 / 2)^{3}\left(v_{d} / V_{A}\right)^{4} \approx 2.10 \times 10^{-4}$. For the background deuterium plasma $s_{m}^{2}=\left(m_{d} / V_{A}\right)^{2} \approx m^{2}(0.0656)^{2} \approx 4.30 \mathrm{~m}^{2} \times 10^{-3} \ll 1$ for $m \ll(0.0656)^{-1} \approx 15.24$. Thus the background deuterium plasma is totally black and opaque for the fundamental, is gray for the second harmonic, 16 and is optically thin for the third and all other higher harmonics. In Eqs. (48) and (51), for our rough estimates, we may write $\left(S_{m}^{-1} \partial\left\{\left[S_{m} J_{m}^{\prime}\right]^{2}\right\} / \partial S_{m}\right)=\left(2 \partial\left\{\left[S_{m} J_{m}^{\prime}\right]^{2}\right\} / \partial S_{m}^{2}\right) \approx\left[\left(2 / S_{m}^{2}\right) \quad\left\{\left[S_{m} J_{m}^{\prime}\right]^{2}\right\}\right] \approx 2$ and $\left(S_{m}^{-1} \partial\left\{\left[S_{m} J_{m}\right]^{2} / 2\right\} / \partial S_{m}\right) \approx 1$, since the maximum values of $J_{m}$ and $J_{m}^{\prime}$ are of order one. Then for fusion product protons, we obtain from these equations that 
$\tau=\left(\mathrm{Y}_{\mathrm{p}} / \mathrm{m}\right)=(\eta \mathrm{Y} / \mathrm{m})=\left[1.121 \times 10^{2} \times \eta / \mathrm{m}\right]$. Thus for $\eta<10^{-2}, \tau<1$. Hence in present experiments, the contribution from fusion product protons to the optical depth $\tau$ can be neglected. In $\mathrm{E}_{\mathrm{i}}$. (58) the factor $\left[\left(\mathrm{g}_{\mathrm{p}}^{2} / 8\right)\left(\hbar \mathrm{k} / \mathrm{M}_{\mathrm{p}} \mathrm{V}_{\mathrm{A}}\right)\right] \approx\left[\left(\mu^{2} / 2\right)\left(\mathrm{g}_{\mathrm{p}} / 2\right)^{3}\left(\hbar \omega_{\mathrm{cp}} / \mathrm{M}_{\mathrm{p}} \mathrm{c}^{2}\right)\right] \approx 2.165 \times 10^{-12}$, where we have made use of Eq. (18) and the relation $\mu=c / V_{A}$. Thus we conclude from Eq. (58) that the optical depth $t$ for the proton spin-flip emission and absorption is always considerably less than unity. Hence the spin-flip emission cannot build up to its black-body value.

\section{E. Estimates of beam driven cyclotron overstability conditions}

It is clear from Eq. (61) that if the total distribution function $f\left(v_{\perp}, v_{\perp}\right)$ can be decomposed as a product $f_{\perp}\left(v_{\perp}\right) f_{\|}\left(v_{\|}\right)$, then by doing an integration by parts over $d v_{\perp}$ it is relatively easy to show that the sign of the net result for $\gamma$ is not at all sensitive to the sign of the slope $\left[\partial f_{\perp}\left(v_{\perp}\right) / \partial v_{\perp}\right]$ anywhere in the range $0 \leq v_{\perp} \leq \infty$. The physical reason for this is that the particle-wave resonance occurs only in the parallel velocity distribution function and not on the perpendicular velocity distribution function. Thus the instability condition of Eq. (62) primarily comes from the familiar cyclotron overstability terms 18,24 of the conventional hot plasma theory. 24 Thus we need large drift velocities along the parallel (and not the perpendicular) direction to produce overstability conditions. For $\omega \approx m \omega_{c i}$ or $m \omega_{s p}$, we need a large enough drift velocity $V_{z}$ such that $\left(V_{z} / V_{A}\right)>\left(T_{\| j} / T_{\perp i}\right)$ or $\left(T_{v} / T_{S}\right)$ for the cyclotron and the spin-flip instabilities, respectively. For example, with a tangential neutral $100 \mathrm{keV}$ hydrogen beam injection we have $\left(\mathrm{V}_{\mathrm{Z}} / \mathrm{V}_{\mathrm{A}}\right) \approx\left(\mathrm{V}_{\mathrm{b}} / \mathrm{V}_{\mathrm{A}}\right) \approx 0.42$. Then for cyclotron instability we need to have a temperature anisotropy such that $\left(\mathrm{T}_{\| \mathrm{i}} / \mathrm{T}_{\perp \mathrm{i}}\right)<0.42$. It is not clear whether such temperature anisotropies exist in Ohmic and/or auxiliary heated tokamak plasmas. A somewhat similar temperature anisotropy was previously observed in the Model C Stellarator plasmas. 60

\section{F. Estimates of spin-flip laser emission}

In the case of spin-flip resonance, one can have the spin-flip overstability due to drifts as in Eq. (66) or one can have an instability due to a direct inversion of the statistical population of the spinup and the spin-down states as in Eq. (63). Indeed at the quasilinear steady state $F_{S}(m)=\left[f_{S}\left(m_{S}\right)\right.$ $\left.-f_{S}\left(-m_{S}\right)\right] \approx 0$, i.e., the populations of the spin-up and the spin-down states are equal to each other, and the spin-flip excitation energy density is enhanced above its black-body thermal equilibrium value of $\mathrm{KT}$ by the factor $\beta$ of Eq. (77). For the fusion product protons with the thermal spread in energy $T_{p} \approx 60 \mathrm{keV}$, we obtain $\beta \approx \eta \times 1.96 \times 10^{5}$ if all these protons were initially statistically inverted, i.e., $F_{s}^{O} \approx 1$. Since the black-body thermal equilibrium value of $K T$ is independent of $\eta$, it is apparent that this spin-flip laser emission of $\beta \kappa T$ is linear in the fusion product proton density. This result is consistent with the TFTR experimental observation that the "broad continuum 
component" of the spectrum of Fig. 1 does follow the time evolution of the neutron flux over about 2.5 orders of magnitude. Further, we showed earlier that for $T_{S} \approx T_{V} \approx T_{p} \approx 60 \mathrm{keV}$ and $(\Delta \omega / \omega) \approx 1$, the black-body spin-flip emission power $P_{A} \approx 1.48 \times 10^{-6} \mathrm{~W}$. Hence, the spin-flip laser emission power $\mathrm{P}_{\mathrm{SL}} \approx \beta \mathrm{P}_{\mathrm{A}} \approx \eta \times 1.96 \times 1.48 \times 10^{-1} \mathrm{~W} \approx 0.29 \eta \mathrm{W}$. For an $\eta$ of 10-6, $P_{S L} \approx 2.9 \times 10^{-7} \mathrm{~W}$. This is in good agreement with the integrated power in the "broader background continuum component" of the TFTR spectrum of Fig. 1, i.e., we showed earlier that for this Fig. 1, $\mathrm{P}_{\mathrm{BBCC}} \approx 2 \times 10^{-7} \mathrm{~W}$.

It is apparent that there is also a broader background continuum component of the emission spectrum in the older JE'; data of Refs. 3 and 4 which were taken with DD fusion reaction dominated plasmas. Let us now specifically concentrate on the data in Fis 1 of Cottrell, Lallia, et. al. of Ref. 4. For this JET data, $B=2.6 \mathrm{~T}, \mathrm{n}_{\mathrm{d}}=\mathrm{n}_{\mathrm{e}}=1.5 \times 10^{13} \mathrm{~cm}^{-3}, \mathrm{~T}_{\mathrm{i}} \approx 2 \mathrm{keV}$, and $\eta \approx 2 \times 10^{-8}$; compared to the chosen values for the above TFTR estimate as: $B \approx 4.8 \mathrm{~T}$, $\mathrm{n}_{\mathrm{d}}=\mathrm{n}_{\mathrm{e}} \approx 5 \times 10^{13} \mathrm{~cm}^{-3}, \mathrm{~T}_{\mathrm{i}} \approx 5 \mathrm{keV}$, and $\eta \approx 10^{-6}$. It is reiatively easy to show from Eq. (77) that the spin-flip laser enhancement factor $\beta \propto\left(\eta B / n_{d} 1 / 2\right)$. Thus the theoretically expected spin-flip laser power for this JET data is $P_{S L}=\left(2.9 \times 10^{-7} \mathrm{~W}\right) \times\left(2 \times 10^{-8} / 10^{-6}\right)$ $(2.6 \mathrm{~T} / 4.81 \mathrm{~T})(5 / 1.5)^{1 / 2}=5.72 \times 10^{-9} \mathrm{~W}$. From the $2 \mathrm{keV}$ black-body plot of their Fig. 1 , we find that $28<2 \omega_{\mathrm{cd}}<65 \mathrm{MHz}$ and the bandwidth $\Delta \mathrm{f}$ of their receiver as $\Delta f=\left(5 \times 10^{-12} \mathrm{~W}\right) /\left[\left(1.6 \times 10^{-19}\right)\left(2 \times 10^{3}\right) / 2\right]=3.125 \times 10^{4} \mathrm{~Hz}$, where we have used their relation that the black-body power $\mathrm{P}_{\mathrm{BB}}=(1 / 2) \kappa \mathrm{T} \Delta \mathrm{f}$. Here the factor $1 / 2$ comes from the fact that their antenna receives only one polarization. Thus for their plasma conditions, the spinflip frequency $\omega_{\text {sp }}$ lies in the range $78<\left(\omega_{\text {sp }}=5.59 \omega_{\mathrm{cd}}\right)<182 \mathrm{MHz}$. Their data of Fig. 1 only goes up to $100 \mathrm{MHz}$. If we now extrapolate their data to $182 \mathrm{MHz}$ and make a triangular approximation to the broader background continuum component of the spectrum in the above $\omega_{\text {sp }}$ range, we find that the total integrated power $\mathrm{PBBCC}_{\mathrm{B}}=\left(5 \times 10^{-12} \mathrm{~W}\right) \times$ $[(182-78) \mathrm{MHz} / 2] /(\Delta \mathrm{f})=8.32 \times 10^{-9} \mathrm{~W}$. Thus the theoretically expected spin-flip laser power $P_{S L}$ is approximately the same as the extrapolation of their experimentally observed total integrated power $\mathrm{P}_{\mathrm{BBCC}}$ in the broader background continuum component of their spectrum for the spin-flip frequency range of $78<\omega_{\mathrm{sp}}<182 \mathrm{MHz}$. It should be noted that the Alfven wave index of refraction $\mu=c / V_{A} \propto\left(n_{d} 1 / 2 / B\right)$ is approximately the same for both the TFTR and the JET data. For this reason all our estimates apply equally well for both TFTR and JET results if we properly take account of the linear scaling differences arising from: (a) the receiving antenna sizes for single dressed test particle emission estimates, (b) the fractional proton and/or alpha particle population $\eta$ for single dressed test particle and proton spin-flip laser emission estimates, and (c) the deuterium ion temperature $T_{d}$ for black-body emission estimates. 
But in the recent JET data taken with DT fusion reaction dominated plasmas, as reported in Fig. 1 by Cottrell, Bhatnagar, et. al. of Ref. 4, there appears to be no such broader background continuum component up to about $6 \omega_{\mathrm{cd}}-\mathrm{E}$. Since the lowest value of the proton spin-flip frequency $\omega_{\mathrm{sp}} \approx 5.59 \omega_{\mathrm{cd}-\mathrm{E}}$, the proton spin-flip laser emission contribution to the background continuum will occur only for frequencies above about $6 \omega_{\mathrm{cd}}-\mathrm{E}$. Here they state that the central alpha particle density $n_{\alpha}$ is a Hence according to our earlier reasoning, the proton density $n_{p} \approx 10^{-4} n_{\alpha} \approx 10^{-8} n_{e}$. That is, the proton fraction $\eta_{\mathrm{p}} \approx 10^{-8}$. Note that this theoretical estimate is only a factor of two smaller than the experimental value used above for the older JET data. Thus the theoretically expected proton spin-flip laser power for this new JET data is $P_{\mathrm{SL}} \approx\left(5.72 \times 10^{-9} \mathrm{~W}\right) / 2=2.86 \times 10^{-9} \mathrm{~W}$. Unfortunately, it is not possible to get an experimental value for $\mathrm{P}_{\mathrm{SL}}$ from their data of Fig. 1. However, they do give the experimental value of $\mathrm{P}_{\mathrm{ICE}}$ as $3.1 \mu \mathrm{W}$. Hence using this theoretical value, we find that the ratio $\left[\mathrm{P}_{\mathrm{SL}}\right.$ due to protons/PICE due to alpha particles] $\approx\left[2.86 \times 10^{-9} \mathrm{~W} / 3.1 \times 10^{-6} \mathrm{~W}\right] \approx 9.2 \times 10^{-4}$. According to our earlier approximate reasoning based on the ratio [DT cross-section / DD cross-section] as $10^{2}$ and the burn up fraction of $1 \mathrm{MeV}$ tritons as $10^{-2}$, we expected this ratio $\left[\mathrm{P}_{\mathrm{SL}}\right.$ due to protons / $\mathrm{PICE}$ due to alpha particles] to be of the order of $10^{-4}$ for DT fusion reaction dominated plasmas. This approximate agreement between half theory and half experiment for this new JET data is comforting and illustrates the selfconsistency of our reasoning.

It may be noted from Eq. (23) that at thermodynamic equilibrium $F_{s}(m)=\left[f_{s}\left(m_{s}\right)-f_{s}\left(-m_{s}\right)\right]=$ $\left[2 \cosh \left(\hbar \omega_{s p} / 2 \kappa T_{s}\right)\right]^{-1}\left[\exp \left(-m_{s} \hbar \omega_{s p} / \kappa T_{s}\right)-\exp \left(m_{s} \hbar \omega_{s p} / \kappa T_{s}\right)\right]=(1 / 2)\left(-2 m_{s} \hbar \omega_{s p} / \kappa T_{s}\right)=$ $\left(-m_{s} \hbar \omega_{s p} / \kappa T_{s}\right)$ for $\hbar \omega_{s p} \ll x T_{s}$. Thus when $T_{s} \rightarrow \infty, F_{s}(m) \rightarrow 0$. It appears therefore at these large kinetic temperatures where the thermal spread in energy is extremely large compared to the spin-flip energy, the spin system under consideration can in principle flip-flop back and forth between its thermodynamic equilibrium state with $F_{s}(m) \approx\left(-m_{s} \hbar \omega_{s p} / \kappa T_{s}\right)$ and its quasilinear radiative steady state with $F_{s}(m) \approx 0$. Because of the extremely low value of ( $\left.\hbar \omega_{s p} / k T_{p}\right)$, such a flip-flop of the spin system between these two states of minimum entropy production is not unreasonable on the basis of the allowed energy fluctuations in a canonical ensemble and the concentration fluctuations in a grand canonical ensemble of statistical mechanics. 14 If these fluctuation laws of statistical thermodynamics allow such a flip-flop of the spin system, then this process can account for the observed broader background continuum component of the spectrum covering the frequency range for which the proton spin-flip frequency $\omega_{\mathrm{sp}}$ is within the plasma. For our case, the fusion product density $n_{p} \approx \eta \times 5 \times 10^{13} \mathrm{~cm}^{-3}$ and the resonant layer volume near the plasma center $\left(\Delta V_{0}.\right)=2 \pi R_{p} \Delta R_{p} 2 a_{s c}=4 \pi R_{p}^{2} a_{s c}(\Delta \omega / \omega)$ 
$\approx 7.17 \times 10^{7} \times(\Delta \omega / \omega) \mathrm{cm}^{3}$. For the TFTR data of Fig. 1, the experimental value of $(\Delta \omega / \omega) \approx 1$ for the background continuum spectrum. Thus the total number of fusion product protons in this resonant volume is $\mathrm{N} \approx \eta \times 3.585 \times 10^{20}$. Hence $\mathrm{N}^{-1 / 2} \approx \eta^{-1 / 2} \times 5.281 \times 10^{-9}$. The value of $\left(\hbar \omega_{\mathrm{sp}} / \mathrm{KT}_{\mathrm{p}}\right)=\left(\hbar \mathrm{g}_{\mathrm{p}} \omega_{\mathrm{cp}} / 2 \mathrm{KT} \mathrm{p}\right) \approx 1.42 \times 10^{-11}$ for $B \approx 4.813 T$ at $R_{p}$ and $K T_{p} \approx 60 \mathrm{keV}$. Assuming that these fusion product protons behave as a perfect classical gas, it is relatively easy to show 14 that the canonical ensemble energy fluctuations $f_{e}$ and the grand canonical ensemble concentration fluctuations $f_{n}$ are both given by $f=f_{e}=f_{n}=N^{-1 / 2}$. Since $\eta \ll 1$ we see that the statistical fluctuations $f$ in energy and concentration are both very much larger than the ratio $\left(\hbar \omega_{s p} / K T_{p}\right)$. Hence it appears that the fluctuations laws of statistical mechanics do indeed allow the possibility for the spin system under consideration to flip-flop between its thermodynamic equilibrium state and its quasilinear steady state. From a thermodynamic point of view, both these states are states of minimum entropy production.

\section{ACCESSIBILITY, MODE CONVERSION AND DECAY INSTABILITY}

In this section we now wish to make a few qualitative and semiquantitative remarks on wave accessibility, mode conversion, and parametric decay instabilities. The problem of interest to us here is the emission, absorption, and propagation of cyclotron harmonic electromagnetic waves with frequencies $\omega=\mathrm{m} \omega_{c i}$ which are less than the lower hybrid frequency $\omega_{L H}$. This $\omega_{L H}$ is given by

$$
\left(\frac{1}{\omega_{L H}^{2}}\right)=\left\{\left[\frac{1}{\left(\omega_{c i}^{2}+\omega_{p i}^{2}\right)}\right]+\left(\frac{1}{\omega_{c i} \omega_{c e}}\right)\right)
$$

Further, since the emission of intense harmonics of almost equal intensity for all harmonic numbers $m \leq m_{0}$ where $m_{0} \omega_{c i}=\omega_{L H}$ can only occur if and only if $\left(k_{\perp} \rho_{i}\right)=\left(k_{\perp}<v_{\perp}>/ \omega_{c i}\right)$ is larger than unity, we must primarily consider situations where $k_{\perp}>k_{\|}$. If $k_{\perp}=0$ exactly, then there is only fundamental emission at $\omega \approx \omega_{c i}$ and no emission at any harmonic with $m \geq 2$. Indeed, we are mainly interested in near perpendicular propagation. Thus for $k_{\perp}>k_{\| 1}$ and $\omega \leq \omega_{L H}$, the allowed electromagnetic waves are the fast Alfven waves with the dispersion relation $\omega \approx k V_{A}$. Thus our interest is in the near perpendicular propagating cyclotron harmonic fast Alfven waves. The first question one may ask is that, are these cyclotron harmonic fast Alfven waves accessible for an outside receiving antenna? The receiving antenna is located in a region of zero or low plasma density. The plasma is an inhomogeneous plasma which is located in the inhomogeneous 
(1/R) toroidal tokamak B-field, and the cyclotron resonance layer of interest appears in a region of relatively high density which is deep inside the plasma. We may then ask whether this cyclotron harmonic wave emitted deep inside the plasma will in fact reach the outside antenna or whether the wave will be reflected back into the plasma at some region of intermediate density and thus will never reach the receiving antenna located outside the plasma. If no such intermediate reflection occurs, we shall sagy that the emitting cyclotron resonance layer is accessible to our receiving antenna. Since our interest is for $\omega \leq \omega_{L H}$, we may make use of the lower hybrid accessibility criterion given by Golant 61 as

$$
\left(\frac{c k_{11}}{\omega}\right)^{2}>1+\left\{\frac{\omega_{p e}^{2}\left(\cos ^{2} \varphi+M_{d} / M_{d}\right)}{\left[\omega_{p e}^{2} \cos ^{2} \varphi+\omega_{c e}^{2}\left(\cos ^{2} \varphi+M_{d} / M_{d}\right)\right]}\right\} .
$$

Here, $\varphi$ is the angle between $B$ and $\nabla n$, and it is assumed that $\cos ^{2} \varphi \ll 1$. For $\cos ^{2} \varphi<M_{e} / M_{d}$, Eq. (82) reduces to

$$
\left(\frac{c k_{1}}{\omega}\right)^{2}>1+\left(\frac{\omega_{p e}^{2}}{\omega_{c e}^{2}}\right) \text {. }
$$

while with $\cos ^{2} \varphi>M_{e} / M_{d}$, the condition $\left(c k_{N} / \omega\right)^{2}>2$ becomes sufficient. Stix, 24 in his Eq. (3-39), gives a more stringent condition $\left(\mathrm{ck}_{\|} / \omega\right)^{2}>2\left(1+\omega_{\mathrm{pe}}^{2} / \omega_{\varepsilon e}^{2}\right)$ for $\cos ^{2} \varphi<\mathrm{M}_{\mathrm{e}} / \mathrm{M}_{\mathrm{d}}$, which according to Golant is not necessary.

For cyclotron harmonic fast Alfven waves $\omega \approx k V_{A}$, then Eq. (83) becomes

$$
\left(\frac{k_{\|}}{k}\right)^{2}>\left(\frac{V_{A}}{C}\right)\left(1+\frac{\omega_{p e}^{2}}{\omega_{c e}^{2}}\right) \approx\left(\frac{V_{A}}{C}\right) \approx\left(\frac{1}{\mu}\right) \text { for } \omega_{c e} \gg \omega_{p e} .
$$

For our plasma conditions, the refractive index of the background deuterium plasma is $\mu=28.56$. Hence, Eq. (83) yields $\cos \theta=k_{\|} / k>0.1871$, i.e., $\theta<79.2^{\circ}$. Thus it appears that we need a large enough $k_{\|}$to have the necessary accessibility while we need a large enough $k_{\perp}$ to have $k_{\perp} \rho_{i}$ larger than unity in order to generate almost equal intensity emissions at all harmonic numbers $\mathrm{m}$, where $1<\mathrm{m}<\mathrm{m}_{0} \approx \omega_{\mathrm{LH}} / \omega_{\mathrm{ci}}$. It is interesting to note that $\left(\mathrm{k}_{\|} / \mathrm{k}\right)>0.1871$ implies a Doppler line broadening $\left.(\Delta \omega / \omega)=2 \mathrm{k}_{\|}\left(2 \mathrm{KT} / \mathrm{M}_{\mathrm{p}}\right)^{1 / 2} / \mathrm{kV}_{\mathrm{A}}\right)>0.0851$ for $\mathrm{T}=\mathrm{T}_{\mathrm{p}} \approx 60 \mathrm{keV}$. The experimentally observed $^{3}$ line width in JET is $(\Delta \omega / \omega) \approx 0.05$ and in TFTR it is 0.005 . Thus, it appears that the observed emission may not have large enough $\mathrm{k} \|$ so as to satisfy the necessary condition for the accessibility of the lower hybrid resonance if the thermal spread of the fusion product protons is 
the Brysk's value $T_{p} \approx 60 \mathrm{keV}$. It may also be noted that for $3 \mathrm{MeV}$ protons in a toroidal field of $B \approx 3.628 T$ at $R_{c} \approx R_{p}+a_{p}$, the Larmor radius for TFTR is $P_{p} \approx 6.88 \mathrm{~cm}$. For JET with $B \approx 2.6 T, \rho_{p} \approx 9.6 \mathrm{~cm}$. Hence, the line width $(\Delta \omega / \omega)$ due to the $R^{-1}$ tokamak $B$-field inhomogeneity over the proton's Larmor orbit is $(\Delta \omega / \omega) \approx\left(2 \rho_{p} / R_{c}\right) \approx 0.0423$ since $R_{c} \approx 325 \mathrm{~cm}$ for TFTR. For JET with $R_{c} \approx 3.9 \mathrm{~m},(\Delta \omega / \omega) \approx 0.049$. Thus the observed line width in JET may also be due to this field inhomogeneity. However, in TFTR it is not clear how the observed line width can be lower than that caused by the field inhomogeity over the Larmor orbit.

For our background deuterium plasma conditions, $\left(\omega_{c d}\left(\omega_{c a}\right) \gg\left(\omega_{c d}^{2}+\omega_{p d}^{2}\right)\right.$, where we have used the suffix $d$ in place of the suffix $i$ to denote the deuterium ions. Hence from Eq. (81), $\omega_{\mathrm{LH}} \approx\left(\omega_{\mathrm{cd}}^{2}+\omega_{\mathrm{pd}}^{2}\right)^{1 / 2}$. For the proton cyclotron harmonic emission at the frequency $\omega \approx m \omega_{\mathrm{cp}}$ the emitting cyclotron resonance layer at $R_{c p}$ will be accessible to the receiving antenna in vacuum at $R_{A}$ if this $R_{c p}$-resonance layer is located between the antenna and the lower hybrid resonance layer $R_{L H d}$ for the background deuterium plasma, i.e., for an antenna located in the outer midplane lowfield edge side, $R_{L H d} \leq R_{c p}<R_{A}$. In general, for a cylindrical tokamak plasma (with $B \propto R^{-1}$ ), the emitting cyclotron resonance volume centered at the major and minor radius point $\left(R_{c}, r_{c}\right)$ will be accessible to the outside receiving antenna in vacuum if this resonance volume is located outside the lower hybrid layer, i.e., if the point $\left(R_{c}, r_{c}\right)$ lie in between the lower hybrid layer and the vacuum vessel. The major and minor radius point $\left(R_{c}, r_{c}\right)$ at which the ion density is such that the deuterium lower hybrid resonance layer is equal to the proton cyclotron resonance layer must satisfy the relation

$$
\left[\omega_{c p}\left(R_{c}, r_{c}\right)\right]^{2}=\left[2 \omega_{c d}\left(R_{c}, r_{c}\right)\right]^{2}=\omega_{L H}^{2}\left(R_{c}, r_{c}\right)=\left[\omega_{c d}^{2}\left(R_{c}, r_{c}\right)+\omega_{p d}^{2}\left(R_{c}, r_{c}\right)\right]
$$

Hence for near perpendicular (i.e., $\theta \approx \pi / 2$ ) emission to be accessible to the outside receiving antenna, most of the emission must come from the critical ion density layer $n_{i}\left(R_{c}, r_{c}\right)$ of Eq. (85). That is, this critical lower hybrid layer of the background plasma deuterium ions $\left(R_{c}, r_{c}\right)$ is such that

$$
3\left[\omega_{c d}\left(R_{c}, r_{c}\right)\right]^{2} \approx\left[\omega_{p d}\left(R_{c}, r_{c}\right)\right]^{2}
$$

Stated differently, the locus of this critical major and minor radius point $\left(R_{c}, r_{c}\right)$ of Eqs. (85) and (85a) defines an "approximately" cylindrical "closed surface shell" at which the background deuterium plasma dielectric coefficient for Alfven waves $K_{A}=\mu^{2} \approx\left[4 \pi n_{i}\left(R_{c}, r_{c}\right) M_{d} c^{2} / B^{2}\left(R_{c}\right)\right]=\left[\omega_{p d}\left(R_{c}, r_{c}\right) / \omega_{c d}\left(R_{c}\right)\right]^{2} \approx 3$. This is illustrated in Fig. 3 . All the near perpendicular emission from regions within this "closed surface shell" will not be 
accessible to the receiving antenna located in the outside vacuum, and only those near perpendicular emissions from plasma regions outside this "closed surface shell" are accessible to the receiving antenna. Thus it is quite possible that most of the observed emission comes from the regions near this "critical resonance layer closed surface shell" defined by the locus of the point $\left(R_{c}, r_{c}\right)$ as a black-body emission for the fundamental and as single particle spontaneous emission for harmonic numbers $m \geq 2$. Since for deuterium ions $K_{A}=\mu^{2} \approx 815.7$ for $n_{i}=5 \times 10^{13} \mathrm{~cm}^{-3}$ and $B=4.183 \mathrm{~T}$ at $R_{p}, \mu^{2} \approx 3$ implies that the outer midplane critical layer $R_{c}$ is at the very outer edge of the plasma at which the ion density has fallen to about $n_{i}\left(R_{c}\right)=1.05 \times 10^{11} \mathrm{~cm}^{-3}$. Here, we have used the fact that $\mu^{2} \propto\left[n_{i}\left(R_{c}, r_{c}\right) / B^{2}\left(R_{c}\right)\right] \propto\left[n_{i}\left(R_{c}, r_{c}\right) R_{c}^{2}\right]$. For example, taking the edge plasma density at $R_{p}+a_{p}$ as $n_{i} \approx 1.0 \times 10^{12} \mathrm{~cm}^{-3}$ and an e-folding distance of about $2.5 \mathrm{~cm}$ for the scrape-off layer plasma, $n_{i}\left(R_{c}\right)=1.05 \times 10^{11} \mathrm{~cm}^{-3}$ yield that the resonant layer is located inside the scrape-off region at about $5.65 \mathrm{~cm}$ from the outer midplane plasma edge. Indeed, for the TFTR data of Fig. 1, we find that the apparent emission location is about $4.8 \mathrm{~cm}$ on the low-field side of the outer midplane plasma edge, in agreement with the accessibility predictions of Eq. (85a). Of course, Eq. (85a) gives us the entire "critical resonance layer closed surface shell." But it does not tell us that the resonant layer should lie only in the outer low-field side midplane plasma edge.

We emphasize that according to Golant 61 and Stix, 24 all the radiations that are emitted by the fusion products located inside this "critical lower hybrid resonance closed shell" with $k_{\|} / k<0.1871$ (i.e., the near perpendicular radiations in the range of $79.2^{\circ}<\theta<90^{\circ}$ ) cannot come out of this closed shell, and are trapped inside this approximately cylindrical lower hybrid clostd shell. They crisscross the main body of the enclosed plasma interior undergoing multiple reflections at this shell boundary, and hence probably build up to the thermodynamic equilibrium black-body value even if $\tau<1$. However, they cannot escape out of this shell, and hence cannot reach the antenna. But the wide angle radiations with $k_{1 /} k>0.1871$ (i.e., $0<\theta<79.2^{\circ}$ ) will not be trapped and pass easily through this lower hybrid shell and reach the receiving antenna. It is well-known 16 that most of the cyclotron emission power or intensity is contained in the regions near $k_{\|} \approx 0$, and indeed, most of the cyclotron harmonic emission occurs with $k \approx k_{\perp}$. In essence, these wide angle radiations are simply the wings of the Doppler broadened near perpendicular emission lines. These escaping wide angle radiations will contribute to the "broader background continuum" component of the spectrum. Since $\tau<1$ for harmonic numbers $m>2$, these radiations are the wings of the single "dressed" test particle emission. Consequently, their contribution to the "broad background continuum" will also be linear with the fusion product density, in agreement with the experimental observations in TFTR. Any radiation that originates 
outside this lower hybrid resonance shell and propagates outward towards the vacuum plasma boundary will not encounter any lower hybrid accessibility problem.

But the outstanding question concerns the observed localization of the ICE source only at the outer midplane edge where the local fusion rate is negligible. It is shown elsewhere 62 that the radial birth profiles of the fusion products $n_{p}(r)$ are centrally peaked, and may be approximated as

$$
n_{p}(r)=n_{p}(0)\left(1-\frac{r}{a_{p}}\right)^{4 / 3} \exp \left[\left(\frac{-20}{3}\right)\left(\frac{r}{a_{p}}\right)^{2}\right]
$$

Hence there are no fusion products born in the scrape-off layer where $a_{p} \leq \mathrm{r} \leq \mathrm{a}_{2 c}$. However, according to Stringer, 62 for typical tokamak parameter conditions, about half the fusion products are formed with pitch angles in velocity space such that they are magnetically mirror trapped (i.e., with $v_{\perp}>v_{\|} \varepsilon^{1 / 2}$, where $\varepsilon=\Delta B / B=r / R$ is the mirror ratio). The radial drift excursions of the banana orbits of these trapped particles can exceed the width of the production profile by considerable amounts. Using Stringer's theory, very recent calculations ${ }^{4}$ for JET by Cottrell, Bhatnagar, et.al. reveal a class of centrally born fusion products (i.e., approximately 10\% centrally born within a narrow range of pitch angles just beyond the trapped-passing boundary) which make large radial excursions, sufficient to reach the outer midplane edge where the velocity distribution is anisotropic and nonmonotonic. These particles make drift excursions only to the low-field side edge. Thus in Fig. 3, the trapped banana orbits of about $10 \%$ of the centraily born fusion products pass through the outer midplane resonance volume $(\Delta \mathrm{Vol})_{0}$. The bottom volume $(\Delta \mathrm{Vol})_{\mathrm{b}}$ should see a small group of lost fusion products leaving the plasma, while the rest of the plasma volume outside the lower hybrid layer which is accessible to the (outside) receiving antenna in vacuum has no fusion products traversing them. That is, for example, the inner midplane volume $(\Delta \mathrm{Vol})_{\mathrm{i}}$, the top volume $(\Delta \mathrm{Vol})_{\mathrm{t}}$, etc. of Fig. 3 all have no fusion products drifting through them. Further, the volume of the background plasma contained in these regions are: $(\Delta V o l)_{0}=4 \pi\left(R_{p}+a_{p}\right)^{2}\left(a_{c}^{2}-a_{p}^{2}\right)^{1 / 2}(\Delta \omega / \omega) ;(\Delta V o l)_{i}=4 \pi\left(R_{p}-a_{p}\right)^{2}\left(a_{c}^{2}-a_{p}^{2}\right)^{1 / 2}(\Delta \omega / \omega) ;$ and $(\Delta \mathrm{Vol})_{\mathrm{t}}=(\Delta \mathrm{Vol})_{\mathrm{b}}=2 \pi \mathrm{R}_{\mathrm{p}}^{2}\left(\mathrm{a}_{\mathrm{ac}}-\mathrm{a}_{\mathrm{p}}\right)(\Delta \omega / \omega)$, respectively. For the tokamak parameters quoted earlier, we find that $(\Delta \mathrm{Vol})_{0}=3.88(\Delta \mathrm{Vol})_{\mathrm{i}}=12.02(\Delta \mathrm{Vol})_{\mathrm{t}}=12.02(\Delta \mathrm{Vol})_{\mathrm{b}}$. Thus the outer low-field side midplane volume ( $\Delta \mathrm{Vol})_{0}$ of Fig. 3 is not only the largest accessible volume, but is the only volume that has an appreciable portion (i.e., approximately 10\%) of the centrally bom fusion products traversing it, while all the other accessible volumes contain no significant amount of fusion products drifting through them. Thus, one can clearly understand the localized nature of ICE in tokamaks to the outer midplane edge. Further, we have shown earlier that the theoretically expected value of $R_{c}$ for the TFTR data of Fig. 1 is $R_{c} \approx\left(R_{p}+a_{p}+5.65\right) \mathrm{cm}$, while the 
experimentally measured value is $R_{c} \approx\left(R_{p}+a_{p}+4.80\right) \mathrm{cm}$, yielding good agreement between theory and experiment.

Based on such accessibility analysis of the lower hybrid resonance and the mode conversion calculations of Stix, 8 Ono ${ }^{10}$ has recently suggested that this ICE may be looked upon as an inverse problem to the familiar ion Bernstein wave heating (IBWH). His qualitative arguments are as follows: The slow, short wavelength electrostatic ion Bernstein waves (IBW) are excited by the hot ion population in the plasma interior which then propagate across the magnetic field towand the low field region of decreasing density. The wave then encounters the critical lower hybrid resonance layer at $\mathbf{R}_{\mathbf{c}}$ at which it undergoes a mode conversion into the fast, long wavelength electromagnetic ion cyclotron harmonic wave which then propagates towards the antenna. Therefore, he suggests that the tokamak IBW accessibility physics is acting as a filter to connect the externally measured electromagnetic signal to the IBW generated in the hot plasma interior. Since the IBW is generated in the interior where the B-field is higher, we must speculate that the received signal at the antenna at a given ion cyclotron harmonic frequency is actually generated in the plasma interior one major harmonic below in order to have the right frequency matching at the critical resonance layer $R_{c}$. This mode conversion interpretation may be appealing to explain the spatially localized nature of the observed emission. However, for ion Bernstein wave heating (IBWH), Ono then states that if the antenna is placed significantly away from the midplane (poloidal angle $\geq 30^{\circ}$ ), the launched parallel wave number increases as the wave propagates towards the midplane (toward decreasing magnetic field). Ray-tracing calculations indicate that a significant up-shift of the parallel wave number occurs in this situation causing the wave to be absorbed via electron Landau damping not too far from the plasma edge. By Kirchhoff's law, it would then appear that ICE should come from all around the plasma edge, and not necessarily localized to the outer midplane plasma edge. In any case, in order to excite these IBW to large amplitudes by the hot ion population in the plasma interior, we must satisfy the instability and/or overstability conditions of Eqs. (61) or (62) with the appropriate phase velocity of these IBW. These conditions may not be so easy to satisfy. Further, it is extremely difficult even to estimate the nonlinear saturated level of such unstable IBW. It is also not clear why such a modeconversion emission ICE power should vary linearly with the fusion product number density. Further, in our view, when appreciable tunneling is needed the present calculations of mode conversion efficiencies are not very reliable. In any case, more accurate mode conversion efficiency modelling is needed before we can say anything more about this. We also wish to point out that, if the fusion products excite the electrostatic IBW in the plasma interior, these electrostatic waves could be detected by microwave or laser scattering experiments in the near future. 63 
For the sake of completeness, we wish to mention that parametric decay instabilities can in principle also account for some of the observed ICE spectrum. Parametric instabilities have been observed in lower-hybrid, 64 ICRF, 65 and other 66 experiments. In general, these instabilities have a low power threshold and tend to occur in the plasma edge regions. In this decay instability the pump wave of frequency $\omega_{0}$ and wave vector $k_{0}$ usually decays into two daughter waves of frequencies $\omega_{1}$ and $\omega_{2}$ and wave vectors $k_{1}$ and $k_{2}$, respectively, such that the energy and momentum conservation relations

$$
\hbar \omega_{0}=\hbar \omega_{1}+\hbar \omega_{2}
$$

and

$$
\hbar k_{0}=h k_{1}+h k_{2}
$$

are simultaneously satisfied. Usually, the electrostatic waves have shorter wave lengths, thus larger $\mathbf{k}$ values, while the electromagnetic waves tend to have longer wave lengths, thus very small $\mathbf{k}$ values. Indeed, one can take the $\mathbf{k}$ value of the electromagnetic wave in the decay process as zero if the other two waves involved in the decay are electrostatic waves. Thus for example, a parent (i.e., pump) electrostatic ion Bernstein wave (ESIBW) can in principle decay into another daughter ESIBW with almost the same $k$ and an electromagnetic ion cyclotron harmonic fast Alfven wave with $k \approx 0$. Such a decay will be allowed by the conservation relations of Eq. (87) if the power threshold conditions are satisfied. Thus some portion of the observed electromagnetic ICE spectra may be a result of the decay of large amplitude ESIBW driven unstable by the hot ion population or by the injected neutral beams. Hence an unstable large amplitude ESIBW can give rise to the emission of an electromagnetic ion cyclotron harmonic wave either by the mode conversion process or by the parametric decay process. Without any analytic calculations, which are rather complex, it is not possible for us to say anything about the relative importance of these two competing processes at the present time.

We wish to point out that since the dispersion relation of these cyclotron harmonic fast Alfven waves is $\omega \neq k V_{A}$ a parent wave at the proton cyclotron frequency $\omega_{c p}$ can decay into two daughter waves both at the deuteron cyclotron frequency $\omega_{c d}$, i.e., $\omega_{c p} \rightarrow \omega_{c d}+\omega_{c d}$. Such a decay will satisfy the energy and momentum conservation relations of Eqs. (87a) and (87b), simultaneously. Similarly, the decays $2 \omega_{c p} \rightarrow 3 \omega_{c d}+\omega_{c d} n \omega_{c p} \rightarrow(2 n-1) \omega_{c d}+\omega_{c d}$ etc. are all allowed by Eqs. (87a) and (87b). This type of parametric decay may account for the sequence of peaks at frequencies $(n+1 / 2) \omega_{\text {cp-E }}$ in TFTR ohmic ICE experiments. Thus from the parent emission lines at $n \omega_{c p}$, one can obtain daughter emission lines not only at $(n+1 / 2) \omega_{c p}$, but also at 
n $\omega_{\text {dd }}$ by such cascading parametric decay processes. These decay processes are possible when the fusion product protons are immersed in the background deuterium plasma.

In the literature, other authors 65 have proposed various different physical mechanisms for the generation of cyclotron subharmonic frequency waves. Fredricks has considered a secondary peak in $f(v)$ as a function of $v_{\perp}$; that is a peak at $v_{\perp}=b>0$, can provide a mechanism for wave generation at $\omega=(n+1 / 2) \omega_{c}$. Gruber et.al. have shown that a bi-Maxwellian distribution having $T_{\perp} \gg T_{\|}$can drive the well-known Harris instability near $\omega=(n+1 / 2) \omega_{c}$. However, their work indicates that extremely large ratios $\left(T_{\perp} / T_{11} \geq 30\right)$ may be required. Porkolab has considered nonlinear ion-cyclotron (Landau) damping to explain the ion-Bernstein wave heating in magnetically confined fusion plasmas. Here, one of the beat waves is at the subharmonic of the ion cyclotron frequency. Abe et.al. have invoked particle trapping as the physical mechanism for resonant heating of plasmas due to cyclotron subharmonic frequency waves. According to Kirchhoff's law, if the plasma can absorb energy at the cyclotron subharmonic frequency, it must then be able to emit at these same frequencies.

\section{CONCLUSIONS AND SUMMARY}

In summary, we have presented a rather comprehensive analysis of the power emission by the fusion product protons, alpha particles, and/or the background deuterium plasma ions not only in their ion cyclotron range of frequencies (ICRF) but also at the proton spin-flip resonance frequency for some specific model velocity-space distribution functions. We have presented a complete theory of spontaneous emission (based on the Einstein A coefficient) from "dressed" test particles, and then examined in sufficient detail the linearized theory of the radiative steady state and/or radiative equilibrium. We have also derived the necessary and sufficient conditions for radiative instability and/or overstability. These conditions apply both for the electromagnetic cyclotron harmonic fast Alfven waves and for the slow electrostatic ion Bernstein waves. In the former case the phase velocity $(\omega / k)=V_{A}$ the Alfven velocity, while in the latter case the phase velocity $(\omega / k) \approx\left(m \omega_{c i} / k_{\perp}\right)=m\left(\omega_{c i} \rho_{i} / k_{\perp} \rho_{i}\right)=m\left(2 K T_{i} / M_{i}\right)^{1 / 2}\left(k_{\perp} \rho_{i}\right)^{-1} \approx m\left(2 \kappa T_{i} / M_{i}\right)^{1 / 2}$, since $k_{\perp} \rho_{i}$ is of order unity for IBW of interest. Then we give the conventional and rather satisfactory quasilinear and/or nonlinear theory of the radiative steady state. By conventional we mean the system is shown to evolve in time by a coupled set of nonlinear master equations, one of which describes the time evolution of the photon distribution function and the other of which describes the time evolution of the particle distribution function via a Fokker-Planck type equation containing the usual diffusion and the dynamical friction type terms. We make some general comments on the types of instabilities, mainly relating to the distinctions between the "kinetic or causal instabilities" 
and the "hydrodynamic instabilities." Indeed, our discussions of the necessary and sufficient conditions for instability and/or overstability are totally restricted to the kinetic instabilities only. We have also presented some numerical estimates for typical tokamak parameters. Here we show that the predictions of our theory are in reasonable agreement with the experimental observations in TFTR and JET. In all cases, we examine not only the ion cyclotron harmonic emission and absorption but also the fusion product proton's spin-flip emission and absorption. Here, we have made use of the well-known "master equation approach" of nonequilibrium quantum statistical mechanics which is based on the knowledge of the fundamental Einstein A and B coefficients and the principle of detailed balance. Finally, we have made some brief semiquantitative remarks on wave accessibility, mode conversion, and parametric decay instability. Based on Stix-Golant lower hybrid accessibility condition and Stringer's tokamak fusion products radial profile analysis, we are able to show that the experimentally observed spatial localization of the emitting and/or the mode conversion layer on the low B-field side plasma edge of the tokamak is also in reasonable agreement with the theoretical expectations.

In essence, the bottom line is as follows: The fundamental $(m=1)$ ICE is a black-body emission since its optical depth $\tau>1$ for typical tokamak parameters, and the second $(m=2)$ harmonic ICE is probably a "gray-body emission" since its $\tau$ is of order one, while the higher ( $m>2$ ) harmonics ICE should be single particle spontaneous emission since their $\tau<1$. Single "dressed" test particle emission is of course proportional to the number of emitters while blackbody emission is independent of the number density. The experimentally observed linear correlation between PICE and total neutron flux is consistent with the single "dressed" test particle emission picture. For the higher harmonics to be of equal intensity one must have $\left(k_{\perp} \rho_{i}\right)>1$. This is not true for the background plasma deuterium ions but is definitely satisfied for the fusion product protons and alpha particles, and is marginally true for the neutral beam energy of about $100 \mathrm{keV}$. For the usual tokamak parameters and neutral beam energies, the ion cyclotron harmonic instability and/or overstability conditions are only barely satisfied by the neutral beam ions but are reasonably satisfied by the fusion product protons. Stix-Golant lower hybrid accessibility condition yields that most of the ICE comes from regions where the local ion cyclotron frequency is of the same order as the local ion plasma frequency for the background deuterium plasma ions. For the usual tokamak conditions this implies that ICE originates from regions very near the plasma edge in agreement with the observations. Stringer's tokamak fusion products radial profile analysis reveal a class of centrally born (marginally mirror trapped) fusion products which make large radial excursions, sufficient to reash the outer midplane edge. Thus, on making use of Stix-Golant lower hybrid accessibility theory in conjunction with Stringer's tokamak radial profile analysis of the fusion products, one can clearly understand the localized 
nature of ICE in tokamaks to the outer low-field side midplane plasma edge. The experimentally observed line width is smaller than that predicted by the Doppler broadening from the minimum value of $k \|$ needed for the accessibility of the lower hybrid resonance by the outside receiving antenna for the proton thermal spread $T_{p}=60 \mathrm{keV}$. The observed fine structure (i.e., being split into doublets) of the ICE lines in JET is fully consistent with Trubnikov's emission theory of the ordinary mode. But the bulk of the emission is in the extraordinary mode. Because of the observed highly spatially localized nature of ICE, mode conversion and/or mode transformation and the parametric decay instability are also viable causes for ICE. However we have not presented any quantitative calculations of the power level to which the ESIBW can be excited by the allowed instabilities, mode conversion efficiencies, the required parametric decay instability thresholds, etc., to draw any detailed conclusions regarding the relative importance of these processes for the observed ICE spectrum. Single particle proton spin-flip spontaneous emission cannot account for the observed background continuum spectrum that exists roughly over the frequency range for which the proton spin-flip resonance is within the plasma. However, because of the high temperature of the spin system $\left(T_{p} \approx 60 \mathrm{keV}\right)$ such that $\left(\hbar \omega_{\text {sp }} / k T_{p}\right) \ll N^{-1 / 2}$ where $N$ is the total number of emitters, the thermodynamic fluctuation laws of statistical mechanics do indeed allow this spin system to flip-flop back and forth between the two allowed states of minimum entropy production (i.e., between the thermal equilibrium state and the quasilinear steady state of a spin-flip laser system). This spin-flip laser emission power is in good agreement with that observed in the broader background continuum spectrum of both the TFTR (Fig. 1) and the older JET data. Hence such a flip-flop of the spin system between these two states of minimum entropy production can, in principle at least, account for the observed background continuum spectrum peaking around $\omega_{\text {sp. }}$. This spin-flip laser emission is linear with the proton number density, in agreement with the TFTR observations. Further, unlike the ion cyclotron harmonic emission for which $k_{\perp} \gg k_{\|}$, this thermally excited spin-flip laser emission is not a near perpendicular emission (i.e., is a wide angle emission with $k_{\|} \gg k_{\perp}$ ) and is hence fully accessible to the outside receiving antenna. However, wide angle ICE escaping out of the lower hybrid layer may also play a significant role for the observed background continuum spectrum. This wide angle ICE power should also be linear with the fusion product number density.

Finally, we hope that the rather extensive and comprehensive analysis presented here will serve as an essential and necessary first step towards an eventual full understanding of the observed ICE and spin-flip emissions in tokamak plasmas, even though we recognize the fact that this ICE problem has an extremely close similarity to the theoretically evasive, but not yet fully understood grand old Landauer ECE problem. 11-13 


\section{ACKNOWLEDGMENTS}

This work was supported by the United States Department of energy under Contract No. DE-ACO2-76-CHO3073. The authors thank their experimental and theoretical colleagues at the Princeton Plasma Physics Laboratory for useful comments. Special thanks are due to Professor T.H. Stix, Drs. J. Strachan and S. Zweben for their invaluable comments. We also thank Professor H. Furth, Drs. M. Zarnstorff, R. Wilson, and K.M. Mc Guire for encouraging and clarifying remarks. 


\section{REFERENCES}

1TFR Group, Nucl. Fusion 23, 425 (1983); W. Clark and the TFR Group, Proceedings of the Fourth International Symposium on Heating in Toroidal Plasmas (Rome, 1984), Vol. 1, pp. 385-391.

2D. Buchenauer, D.Q. Hwang, K. McGuire, and R.J. Goldston, Proceedings of the Fourth International Symposium on Heating in Toroidal Plasmas (Rome, 1984), Vol. 1, pp. 111-118. ${ }^{3}$ G.A Cottrell and R.O. Dendy, Phys. Rev. Lett. 60, 33 (1988).

${ }^{4}$ G.A. Cottrell, P.P. Lallia, G. Sadler, and P. van Belle, Proceedings of the Thirteenth European Conference on Controlled Fusion and Plasma Heating (Schliersee, 1986), Vol.2, Part 1, pp 37-40; G. Sadler (private communication), also in The Third Workshop on Alpha Particle Physics, M.I.T., U.S.A. (May 28 - 29, 1992); G.A. Cottrell, V.P. Bhatnagar, O. da Costa, R.O. Dendy, A. Edwards, J. Jacquinot, M.F.F. Nave, M. Schmid, A. Sibley, P. Smeulders, and D.F.H. Start, Europhysics Conference Abstracts, 1992 International Conference on Plasma Physics (Innsbruck, 1992), Vol. 16C, part 2, Poster No. 1-100, vol. 1, p.327, (also private communication).

5G.J. Greene, P.L. Colestock, E.D. Fredrickson, et.al. , Proceedings of the Fifteenth European Conference on Controlled Fusion and Plasma Heating (Dubrovnik, 1988), Vol. 12B, Part 1, pp. 107-110; G.J. Greene and the TFTR Group, Proceedings of the Seventeenth European Conference on Controlled Fusion and Plasma Heating (Amsterdam, 1990), Vol. 14B, Part IV, pp. 1540-1543.

6P. Schild, G.A. Cottrell, and R.O. Dendy, Nucl. Fusion 29, 834 (1989).

${ }^{7}$ G.J. Greene, Proceedings of the Ninth Topical Conference on High Temperature Plasma Diagnostics (Santa Fe, New Mexico, March 15 - 19, 1992).

${ }^{8}$ T.H. Stix, Phys. Rev. Lett. 15, 878 (1965).

${ }^{9}$ D.G. Swanson and V.F. Shvets, Phys. Rev. Lett. 68, 3036 (1992); D.G. Swanson, Plasma Waves (Academic, Boston, 1989), Sec. 6.3.

10M. Ono, Princeton University Plasma Physics Laboratory Report PPPL-2832 (March, 1992).

11G. Landauer, J. Nucl. Energy Pt. C4, 395 (1962).

12G. Bekefi, J.D. Coccoli, E.B. Hooper, Jr., and S.J. Buchsbaum, Phys. Rev. Lett. 9, 6 (1962); S.J. Buchsbaum and A. Hasegawa, Phys. Rev. Lett. 12, 685 (1964).

${ }^{13} \mathrm{E}$. Canobbio and R. Croci, Proceedings of the Sixth International Conference on Ionization Phenomena in Gases, T. Hubert and E. Cremien, Eds. (Bureau des Editions, Centre d'Etudes Nucleaires de Saclay, Paris, 1964), Vol. 3, p. 269; A. Simon and M.N. Rosenbluth, Phys. Fluids 6, 1566 (1963); T.H. Stix, Ref. 8. In sharp contrast to Landauer's anomalous results of 
Ref 11, see the first hot plasma theory verification results of V. Arunasalam, E.B. Meservey, M.N. Gurnee, and R.C. Davidson, Phys. Fluids 11, 1076 (1968). This verification is the genesis of present day measurements of the electron temperature via measurements of the radiation temperature near the second harmonic of the electron cyclotron frequency.

${ }^{14}$ C. Kittel, Elementary Statistical Physics (John Wiley \& Sons, Inc, New York, 1958); Leo P. Kadanoff and G. Baym, Quantum Statistical Mechanics (Frontiers in Physics, W.A. Benjamin, Inc, New York, 1962).

15V. Arunasalam, J. Math. Phys. 10, 1305 (1969).

16G. Bekefi, Radiation Processes in Plasmas (Wiley, New York, 1966).

17B.A. Trubnikov, Magnetic Emission of High Temperature Plasmas, Dissertation, Moscow Institute of Engineering and Physics, Moscow (1958): USAEC Tech. Information Service AEC-tr-4073 (1960).

18V. Arunasalam, Phys. Fluids B 4, 1643 (1992).

19E.G. Harris, A Pedestrian Approach to Quantum Field Theory, (John Wiley \& Sons, New York, 1972), pp. 89 - 90 and $42-43$.

20V. Arunasalam, J. Phys. A7, 2211 (1974).

21 W.B. Thompson, "Kinetic Theory of Plasmas", in Advanced Plasma Theory, M.N. Rosenbluth, Ed. (Academic Press, New York, 1964), pp. 1 - 53.

22 B. Coppi, S. Cowley, R. Kulsrud, P. Detragiache, and F. Pegararo, Phys. Fluids 29, 4060 (1986); N.E. Lindgren, A. Bruce Langdon, and C.K. Birdsall, Phys. Fluids 19, 1026 (1976).

23 5. Tamor, Nucl. Fusion 18,229 (1978); V. Arunasalam, P.C. Efthimion, J.C. Hosea, H. Hsuan, and G. Taylor, Phys. Rev. A36, 3909 (1987); 37, 2063 (1988).

24T.H. Stix, Thie Theory of Plasma Waves, (Mc-Graw Hill, New York, 1962).

25N.A. Krall and A.W. Trivelpiece, Principles of Plasma Physics, (Mc-Graw Hill, New York, 1973), Secs. 8.14 and 11.19; D.C. Montgomery and D.A. Tidman, Plasma Kinetic Theory, (Mc-Graw Hill, New York, 1964), Secs. 10.6 and 14.1; D.G. Swanson, Plasma Waves, (Academic, Boston, 1989).

26J.D. Jackson, Classical Electrodynamics, (John Wiley \& Sons, New York, 1975); W.K.H. Panofsky and M. Phillips, Classical Electricity and Magnetism, (Addison-Wesley, Reading, Mass, 1956); J.L. Synge, Relativity: The Special Theory (North-Holland, Amsterdam, 1958).

27C. Chu and J.L. Sperling, J. Plasma Phys. 40, Pt. 3, 407 (1988).

28V. Arunasalam, Phys. Rev. 158, 234 (1967).

${ }^{29}$ R.O. Dendy, C.N. Lashmore-Davis, and K.F. Kam, AEA Fusion Culham Laboratory, (Euratom / UKAEA Fusion Association) Report, AEA-Fus-126. 
30J.A. Rome, D.G. Mc Alees, J.D. Callen, and R.H. Fowler, Nucl. Fusion 16, 55 (1976).

${ }^{31}$ G.N. Watson, Theory of Bessel Functions (Cambridge University Press, Cambridge, England, 1945) p. 395.

32S. Chandrasekhar, Plasma Physics (University of Chicago Press, Chicago, 1960).

${ }^{33}$ A.J. Dekker, Solid State Physics (Prentice-Hall, Inc, Englewoods Cliffs, New Jersey, 1957);

C. Kittel, Introduction to Solid State Physics (John Wiley \& Sons, Inc, New York, 1956);

F. Bloch, Phys. Rev. 70, 460 (1946).

34J. Schwinger, Quantum Electrodynamics (Dover Publications, Inc, New York, 1958).

35W. Heitler, The Quantum Theory of Radiation (Clarendon Press, Oxford, England, 1954).

36V. Arunasalam, Phys. Rev. A7, 1353 (1973).

37E.G. Harris, J. Nucl. Energy, Pt. C: Plasma Phys. 2, 138 (1961).

38R.C. Davidson and C.S Wu, Phys. Fluids 13, 1407 (1970).

39 J.D. Gaffey, W.B. Thompson, and C.S. Liu, J. Plasma Phys., Pt. 2, 7, 189 (1972).

40H.L. Berk, L.D. Perlstein, J.D. Callen, C.W. Horton, and M.N. Rosenbluth, Phys. Rev. Lett. 22, 876 (1969); A. Bers and S. Gruber, Appl. Phys. Lett. 6, 27 (1965).

41V. Arunasalam, Phys. Rev. Lett. 28, 1499 (1972).

42V. Arunasalam, J. Appl. Phys. 46, 3119 (1975).

${ }^{43}$ C.C. Grosjean, Formal Theory of Scattering Phenomena (Institut Interuniversitaire, Des Sciences Nucleaires, Bruxelles, Belgium, 1960), Monographie No. 7.

${ }^{44}$ C.B. Wharton, J.H. Malmberg, and T.M. O'Neil, Phys. Fluids 11, 1761 (1968); W.L. Kruer, J.M. Dawson, and R.N. Sudan, Phys. Rev. Lett. 23, 838 (1969).

45V. Arunasalam, M.A. Heald, and J. Sinnis, Phys. Fluids 14, 1194 (1971).

46R. Karplus and J. Schwinger, Phys. Rev. 73, 1020 (1948); M.W.P. Strandberg, Microwave Spectroscopy (Wiley, New York, 1954); V. Arunasalam, Phys. Fluids 16, 718 (1973); ibid, J. Appl. Phys. 43, 4838 (1972); 44, 1934 (1973).

47T.H. Dupree, Phys. Fluids 9, 1773 (1966).

48V. Arunasalam, Phys. Rev. 149, 102 (1966).

49V. Arunasalam, Phys. Rev. Lett. 37, 746 (1976).

50J. Busnardo-Neto, J. Dawson, T. Kamimura, and A.T. Lin, Phys. Rev. Lett. 36, 28 (1976).

51 C. da C. Rapozo, A.S. de Assis, and J. Busnardo-Neto, Phys. Rev. A 42, 989 (1990); ibid, Plasma Phys. and Controlled Fusion 33, 875 (1991).

52D. Pines and J.R. Schrieffer, Phys. Rev. 125, 804 (1962); ibid, 124, 1387 (1961); Vadim N.

Tsytovich, Nonlinear Effects in Plasma, (Plenum, New York, 1970).

53C.K.N. Patel and E.D. Shaw, Phys. Rev. Lett. 24, 451 (1970).

54J.E. Drummond, Plasma Physics (Mc Graw-Hill, New York, 1961), Ch. 4. 
55W.E. Drummond and D. Pines, Nucl. Fusion Suppl. Pt. 3, 1049 (1962); E. Frieman and P. Rutherford, Ann. Phys. (N.Y.) 28, 134 (1964); A. Vedenov, E. Velikhov, and A. Sagdeev, Nucl. Fusion Suppl. Pt. 2, 465 (1962).

56V.D. Shapiro, Zh. Eksp. Teor. Fiz. 44, 613 (1963) [Sov. Phys. JETP 17, 416 (1963)]; See also Ref. 42.

57P. Batistoni and Cris W. Barnes, Plasma Phys. Controlled Fusion 33, 1735 (1991).

58D.J. Rose and M. Clark, Jr., Plasmas and Controlled Fusion (John Wiley \& Sons, Inc., New York, 1961), Chs. 2 and 4; John Wesson, Tokamaks (Clarendon Press, Oxford, 1987), Ch. 1. 59 H. Brysk, Plasma Phys. 15, 611 (1973).

60 V. Arunasalam, E.B. Meservey, M.N. Gurnee, and R.C. Davidson, Phys. Fluids 11, 1076 (1968); E.B. Meservey and S.P. Schlesinger, Phys. Fluids 8, 500 (1965).

61 V.E. Golant, Sov. Phys.-Tech. Phys. 16, 1980 (1972).

62T.E. Stringer, Plasmas Phys. 16, 651 (1974); S. Glasstone and R.H. Lovberg, Controlled Thermonuclear Reactions (Van Nostrand, New York, 1960).

${ }^{63}$ H. Park, P.S. Lee, W.A. Peebles, and N.C. Luhmann, Jr., Nucl. Fusion 25, 1399 (1985); H. Park, N.C. Luhmann, Jr., W.A. Peebles, and R. Kirkwood, Phys. Rev. Lett. 52, 1609 (1984); N.C. Luhmann, Jr., in Infrared and Millimeter Waves, Vol. 2 (K.J. Button, Ed., Academic Press, New York, 1979); D.L. Brower, H.K. Park, W.A. Peebles, and N.C. Luhmann, Jr., in Topics in Millimeter Wave Technology, Vol. 2 (K.J. Button, Ed., Academic Press, New York, 1988); V.N. Budnikov, Nucl. Fusion 31, 611 (1991).

${ }^{64}$ M. Porkolab, S. Bernabei, W.M. Hooke, R.W. Motley, and T. Nagashima, Phys. Rev. Lett. 38, 230 (1977); K.L. Wong and M. Ono, Phys. Rev. Lett. 47, 842 (1981).

65 M. Ono, M. Porkolab, and R.P.H. Chang, Phys. Fluids 13, 1656 (1980); R. Van Nieuwenhove, G. Van Ooast, J.M. Beuken, et.al. on Controlled Fusion and Plasma Heating (Proc. 15th Eur. Conf. Dubrovnik, 1988), Vol. 12B, Pt. 2, European Physical Society 889 (1988).

66M. Porkolab, V. Arunasalam, and R.A. Ellis, Jr., Phys. Rev. Lett. 29, 1438 (1972);

M. Porkolab, V. Arunasalam, N.C. Luhmann, Jr., and J.P.M. Schmitt, Nucl. Fusion 16, 269 (1976); M. Porkolab, V. Arunasalam, and N.C. Luhman, Jr., Plasma Phys. 17, 405 (1975).

${ }^{67}$ R.W. Fredricks, J. Geophys. Res. 76, 5344 (1971); S. Gruber, M.W. Klein, and P.L. Auer, Phys. Fluids 8, 1504 (1965); M. Porkolab, Phys. Rev. Lett. 54, 434 (1985); H. Abe, H. Okada, R. Itatani, M. Ono, and H. Okuda, Phys. Rev. Lett. 53, 1153 (1984). 


\section{FIGURE CAPTIONS}

Fig.1. A typical TFTR spectrum showing the ICE lines riding on the top of the broader background continuum emission spectrum. Here, $26.4 \mathrm{MW}$ deuterium neutral beams were injected into a deuterium background plasma. The other associated plasma parameters were: $B=4.45 \mathrm{~T}$ at $\mathrm{R}_{0}=265 \mathrm{~cm}, \mathrm{I}_{\mathrm{p}}=1.78 \mathrm{MA}, \mathrm{R}_{\mathrm{p}}=245.2 \mathrm{~cm}$, and $\mathrm{a}_{\mathrm{p}}=80.1 \mathrm{~cm}$. The vertical axis is the ICE power PICE (in $\mathrm{dbm}$ ) deduced from the toroidal RF magnetic field $\delta B$ at the probe, and the analyzer bandwidth $\Delta f=300 \mathrm{kHz}$. The main sequence of harmonic peaks occur at multiples of $27.13 \mathrm{MHz}$, and the deuteron cyclotron frequency $\omega_{b d}$ at the outer midplane plasma edge, i.e., at $\left(R_{p}+a_{p}\right)$, is $\omega_{c d}=27.54 \mathrm{MHz}$. The apparent emission location in the scrape-off plasma is $4.8 \mathrm{~cm}$ on the midplane low-field side of the outer plasma edge.

Fig.2. A plot of $\left\langle\mathrm{G}_{\mathrm{m}}\right\rangle$ of Eq. (14) as a function of the harmonic number $m$ for the monoenergetic isotropic velocity space spherical shell distribution, i.e., with $\left\langle v_{\perp}\right\rangle=V_{i}$ and $\left\langle v_{\|}\right\rangle=(2)^{-1 / 2} V_{i}:$ Figs.2(a), 2(b), 2(c), 2(d), 2(e) and $2(f)$ are for $\left(V_{i} / V_{A}\right) \approx 0.5$, $1.0,1.5,2.0,2.5$, and 3.0, respectively. Here, $V_{A}$ is the Alfven speed in the background deuterium plasma.

Fig.3. A schematic plot of the plasma geometry showing the plasma region, the scrape-off layer region, the accessibility lower hybrid resonance layer where $\mu^{2}=3$, and four sample emission volumes. Here, $(\Delta \mathrm{Vol})_{0}$ is the outer low-field side midplane sample volume, $(\Delta \mathrm{Vol})_{\mathfrak{i}}$ is the inner high-field side midplane sample volume, $(\Delta \mathrm{Vol})_{t}$ is the top sample volume centered at $R_{p}$, and $(\Delta V o l)_{b}$ is the bottom sample volume centered at $R_{p}$, all for a fixed $\Delta \omega / \omega$ so that $\Delta R=R \Delta \omega / \omega$. 


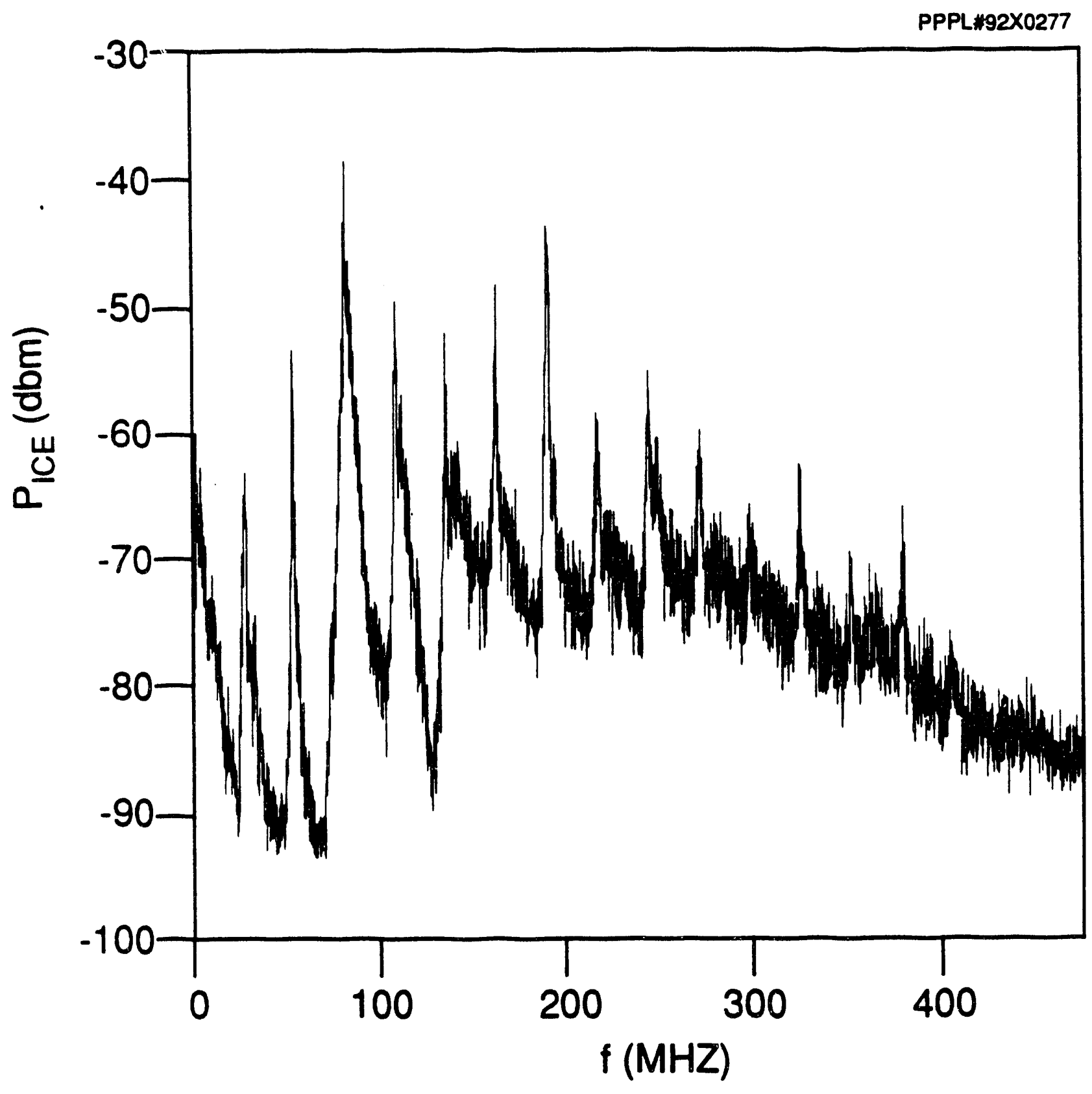

Fig. 1 


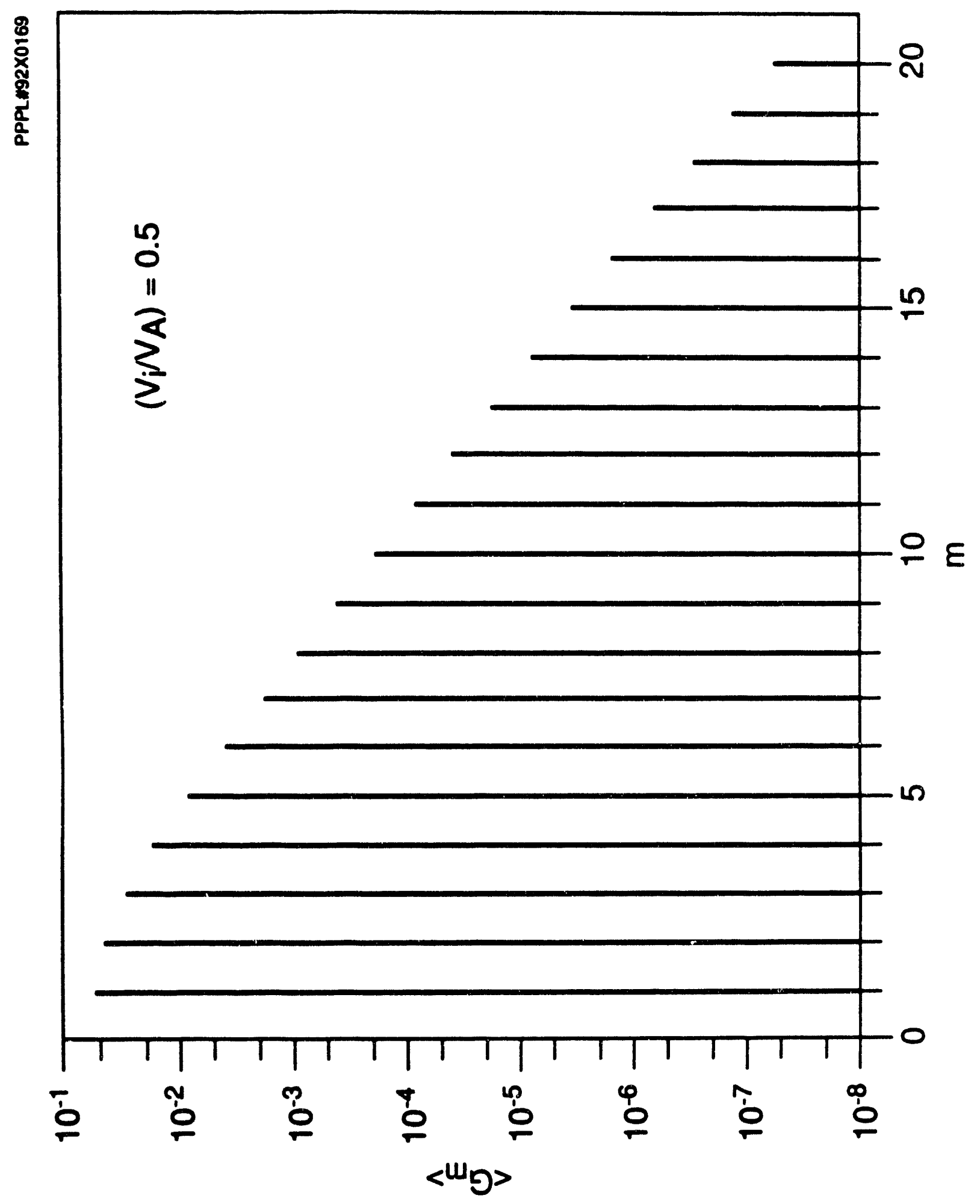

$\underset{2}{*}$ 


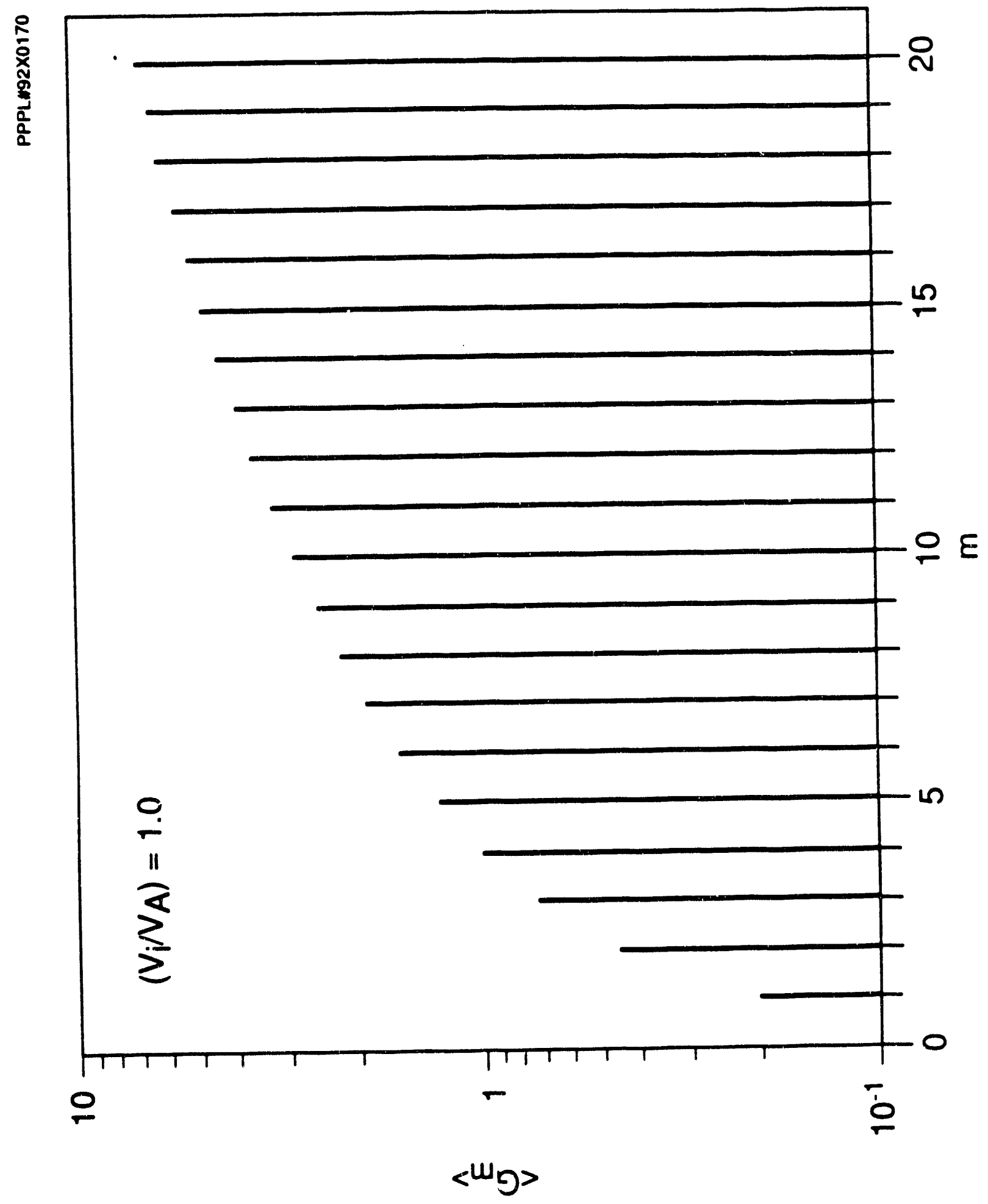




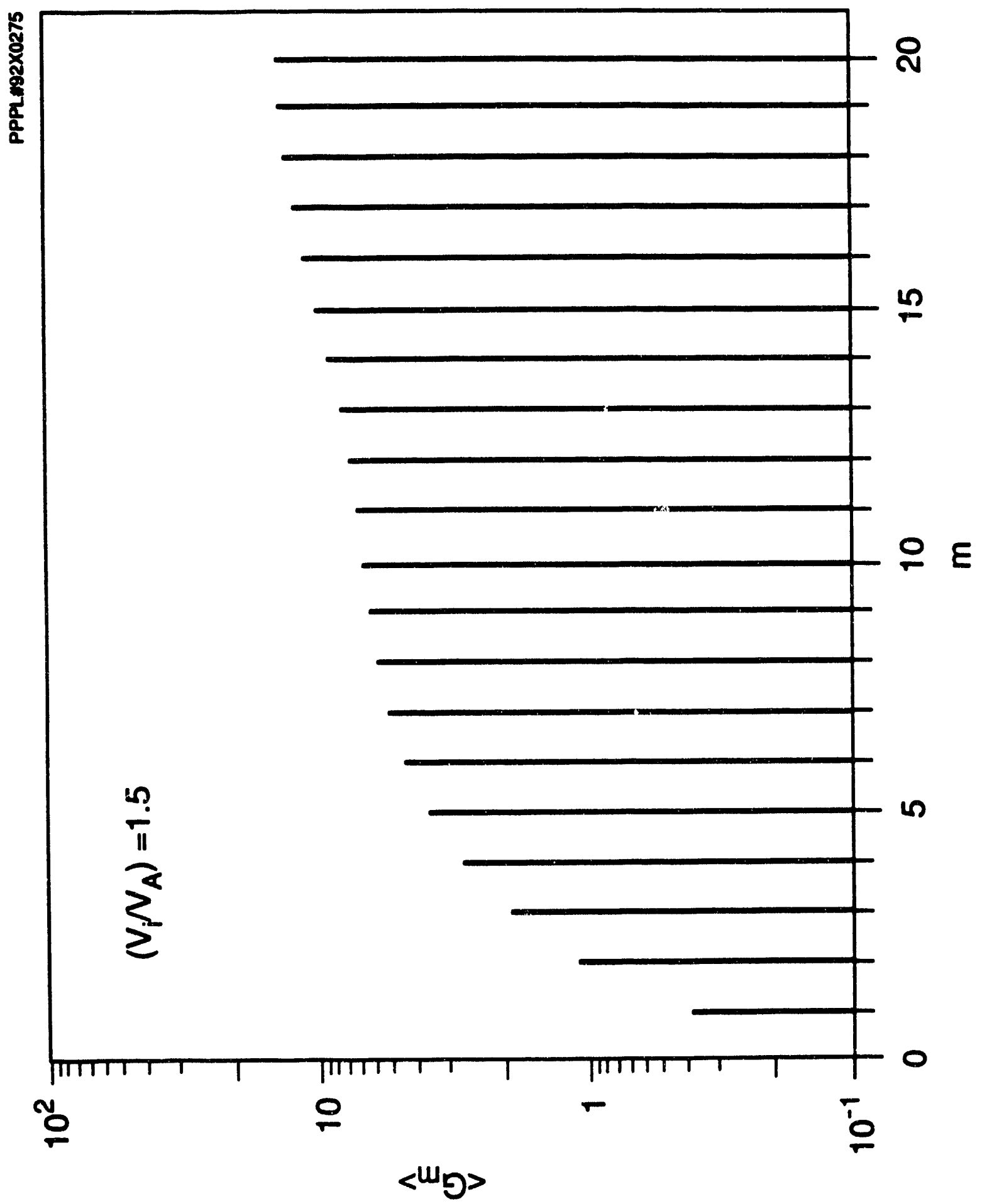



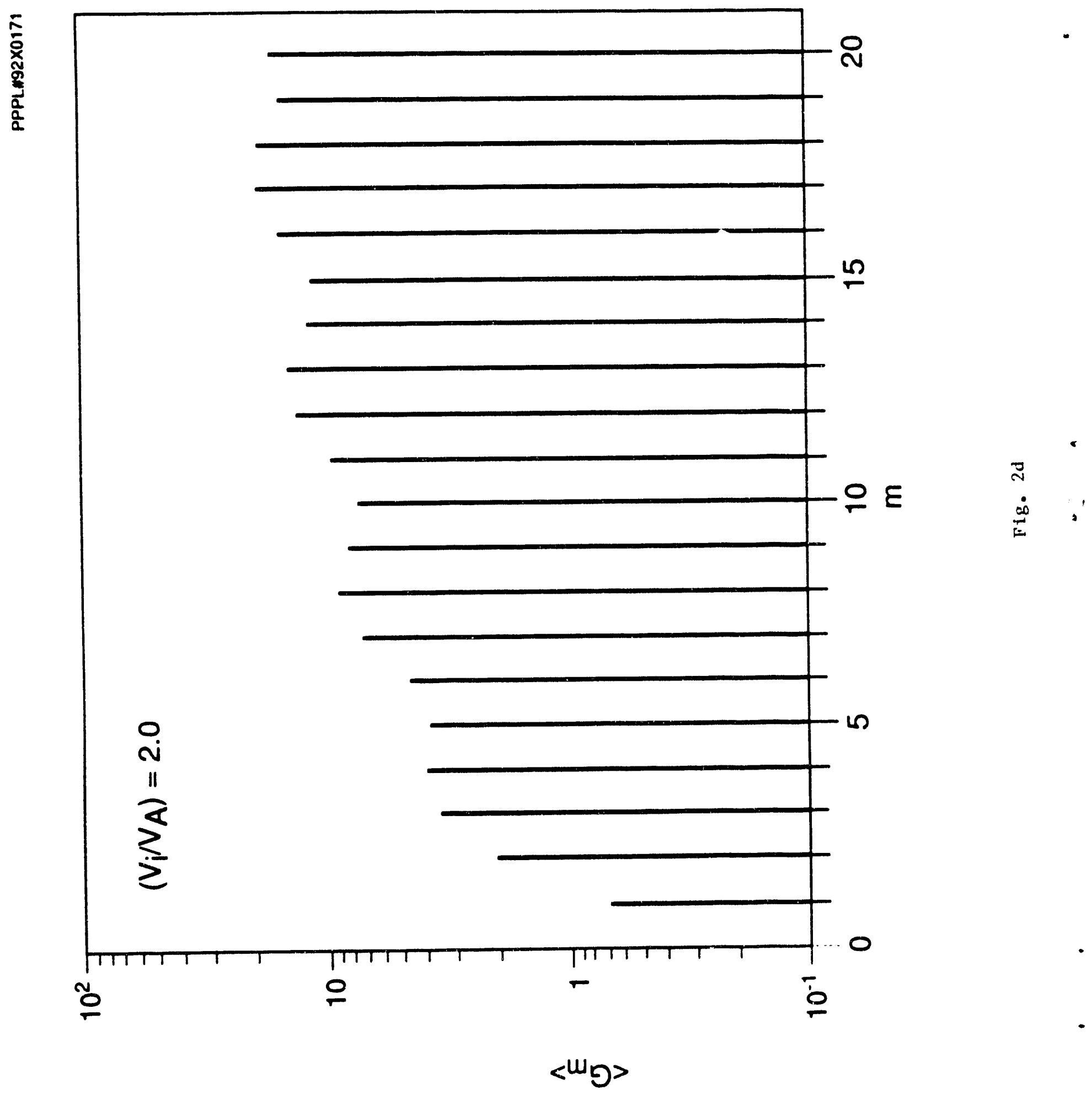


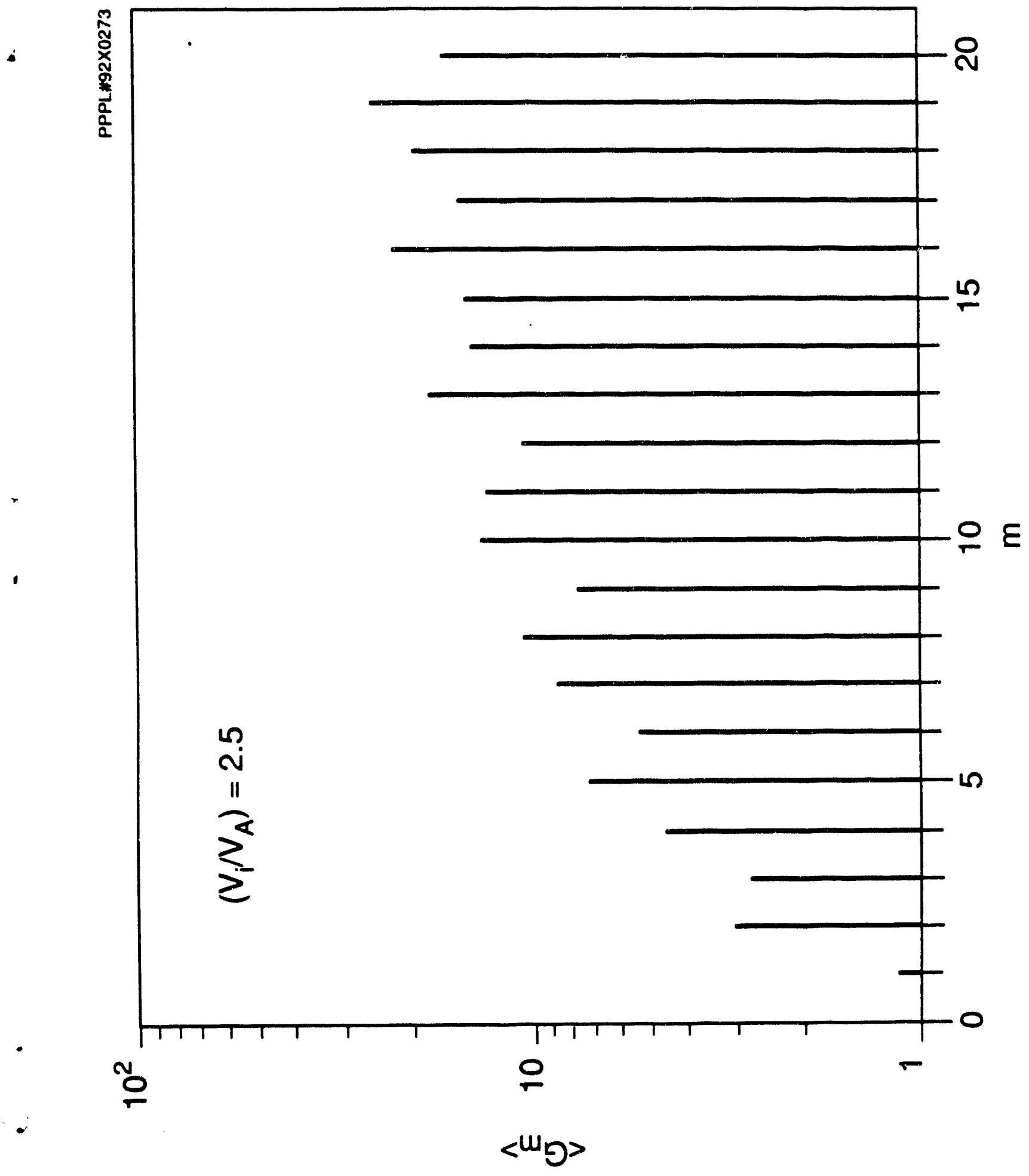

$\stackrel{0}{\sim}$ 


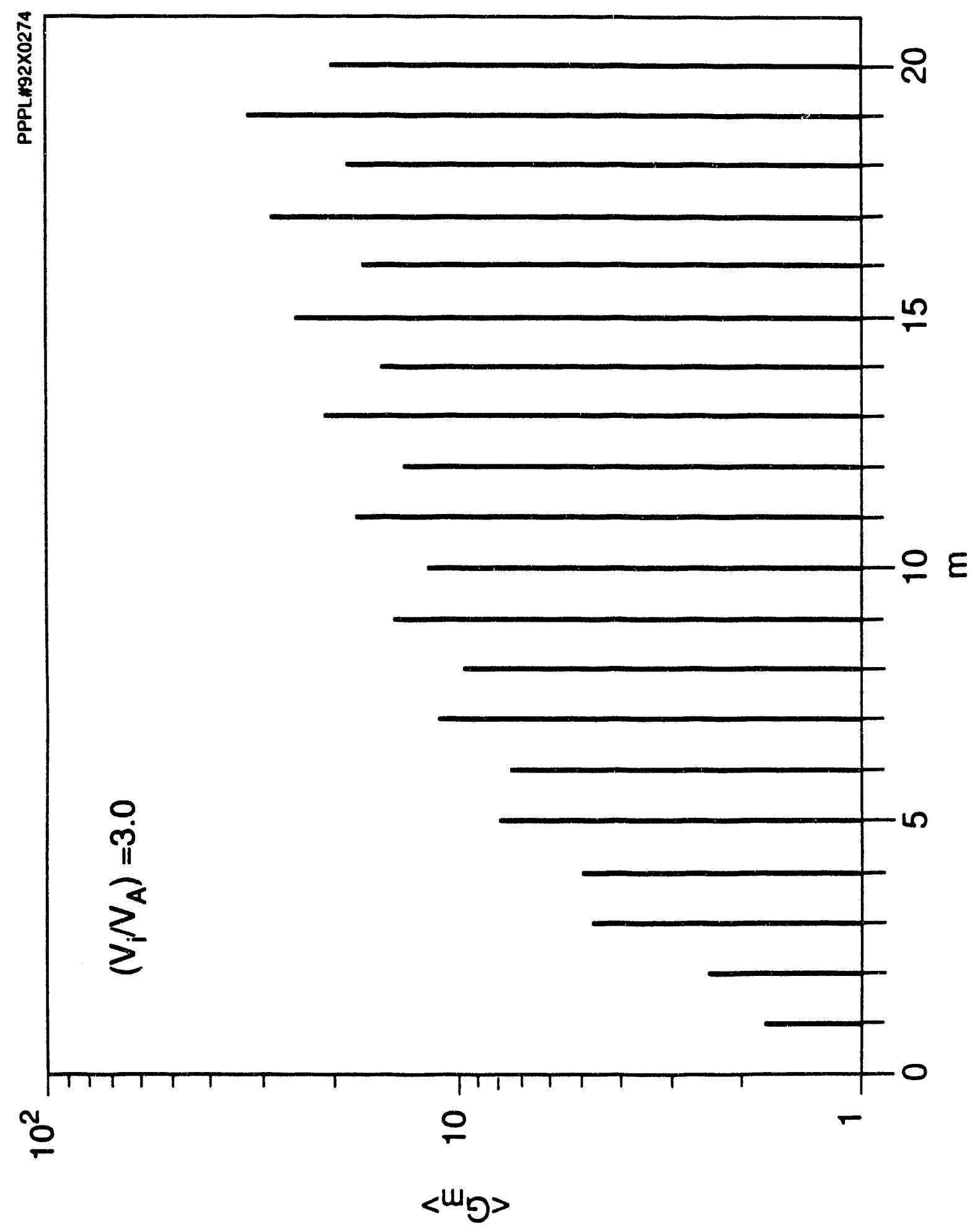

$\stackrel{4}{N}$ 

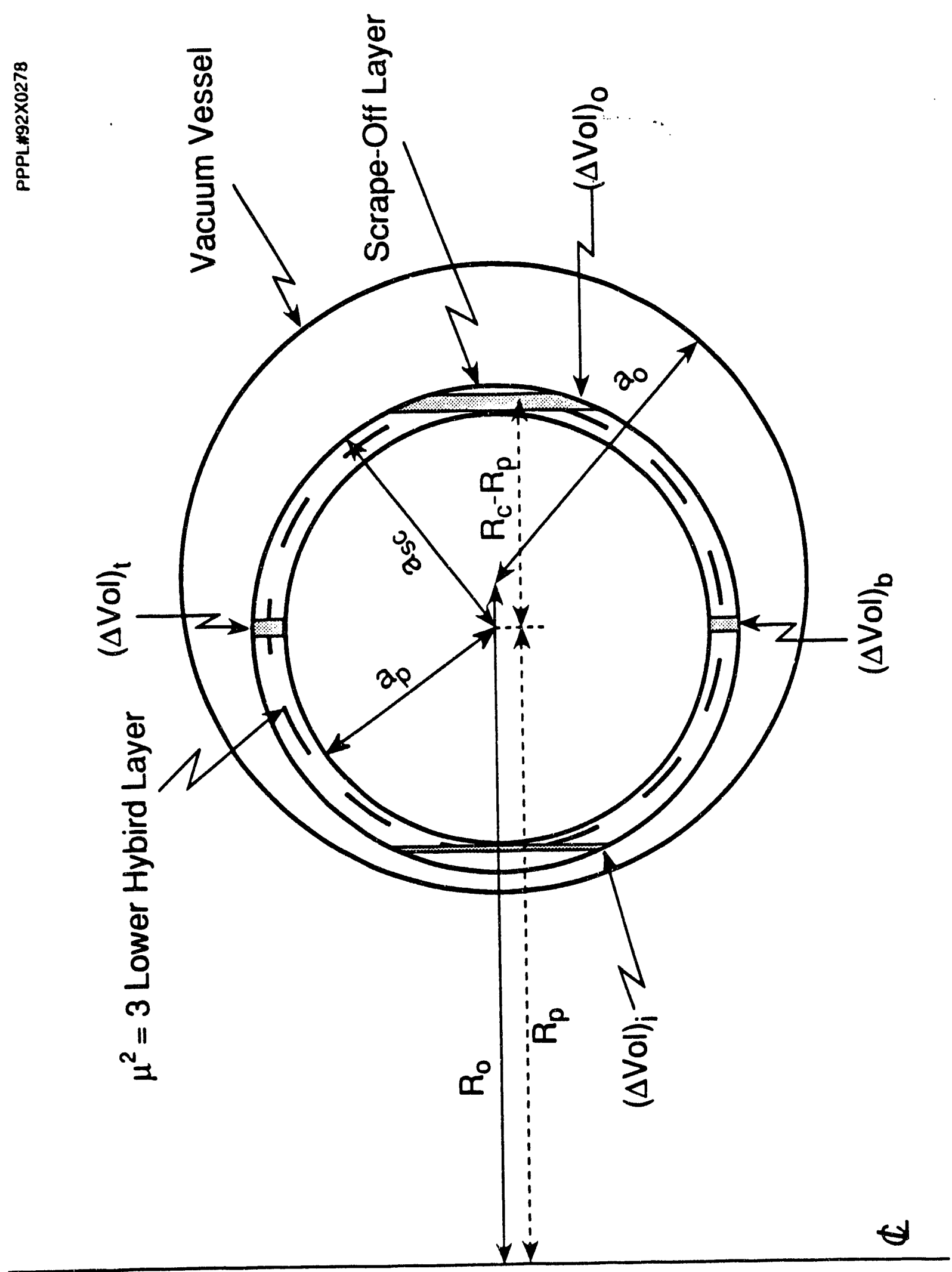
Dr. F. Paoloni, Univ. of Wollongong. AUSTRALIA

Prof. M.H. Brennan, Univ. of Sydney, AUSTRALIA

Plasma Rosearch Lob., Austrelien Nat. Univ., AUSTRALIA

Prof. I.R. Jones, Flinders Univ, AUSTRALIA

- Prof. F. Cap, Inst for Theoreticed Physics, AUSTRIA

Prot. M. Heindier, Inatius for Theorotische Phyeik, AUSTRIA

Prot. M. Goosecens, Aetronomicch Inctiwut, BELGIUM

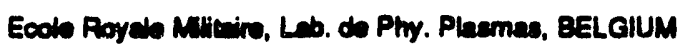

Commiscion-Europaen, DG. XIHFusion Proo., BELGIUM

Prof. R. Bouciqud, R"jkeuniverituit Gent, BELGUM

Dr. P.H. Sukenaka, Instiuto Ficice, BRNZIL

Inetiuto Neciond Do Porquieses Espacicie-INPE, BRUZIL

Documents Orifos, Alomic Eneroy of Cenada Lod., CAMADA

Dr. M.P. Bectyneki, MPB Technologies, Inc., CANADA

Dr. H.M. Skareand, Univ. of Sackatchewen, CANADA

Prof. J. Teichmam, Univ. of Montred, CAMAOA

Prot. S.R. Sreenivacen, Univ. of Colgary, CANADA

Prof. T.W. Jomitan, INASE Energio, CANADA

Dr. R. Boven, Contro canedien de tusion magnstique, CANAOA

Dr. C.R. James, Univ. of Alberta, CANADA

Dr. P. Lukte, Komenektho Untwerezith, CZECHOSLOVAKIA

The Librerien, Culham Laborabry, ENGLAND

Librery. R81, Puthertord Appletion Laboratory, ENGLAND

Mre. S.A. Hurchineon, JET Librery. ENGLAND

Dr. S.C. Shame, Univ. of South Pacific. FIJI ISLANDS

P. Mrtomen, Univ. of Helcinki, FNLAND

Prot. M.N. Busesce, Ecols Polymohniqus., FRANCE

C. Mound, Lot. do Phycique des Milioux loniads, FRANCE

J. Radal, CENCADARACHE - Bat 506, FRANCE

Prot. E. Economou, Univ. of Crom, GREECE

M. C. Rimi, Univ. of loamina, GREECE

Dr. T. Mud, Acadomy Ebliogrephic Sor., HONO KONO

Proprint Librey. Hungerian Acadeny of Sa., HUNGARY

Dr. B. Descuptia, Seha hase of Nuciser Physics, INDIA

Dr. P. Ken, inct. for Pleame Research, INDU

Dr. P. Pocenew, lened inst of Technology, ISRAEL

Libresien, Intemationel Cenver for Theo Phycies, ITALY

Mias C. Do Pab, Associazions EUPATOMENEA, ITALY

Dr. G. Groseo, latituto di Ficica del Plaema, ITALY

Prof. G. Postengni, Istiuns Gas lonizzesi Del Cnr, ITALY

Dr. H. Yemato, Tochiba Res a Dovel Center, JAPAN
Prof. I. Kawakami, Hiroshima Univ., JAPAN

Prof. K. Nishikawa, Hiroshima Univ., JAPAN

Director, Japen Alomic Energy Rosearch Inst, JAPAN

Prof. S. Ibh, Kyushu Univ., LAPAN

Rosearch Into. Cr., Nationd Instit. for Fusion Scienco, JAPAN

Prof. S. Tenaka, Kyob Univ., JAPAN

Librey, Kyoto Univ., JAPAN

Prot. N. Inows, Univ. of Tokyo, LAPAN

Secretery, Pleme Saction, Electrotechnicel Lab., JAPAN

S. Mori, Technicel Advisor, MAERI, LAPAN

Dr. O. Mind, Kumenow Inat of Technology. JAPAN

J. Hyoonsook, Korea Abomic Enorgy Posaarch Inst, KOREA

D.I. Chai, Tho Korea Adv. Inot. of Sai. \& Tech., KOREA

Prot. B.S. Liby, Univ. of We.delo, NEW ZEALAND

Inet of Phyrica, Chinese Aand SA PEOPLE'S REP. OF CHINA

Library, Inat of Plasma Physica, PEOPLE'S REP. OF CHINA

Teinghua Uniw. Libray, PEOPLE'S REPUBLIC OF CHINA

$Z$ Li, S.W. Inet Phycics, PEOPLE'S REPUBLIC OF CHINA

Prof. J.A.C. Contord, Inetuno Superior Tecnico, PORTUGAL

Dr. O. Potrus, Al I CUzA Univ., ROMANIA

Dr. J. do Vitiers, Fucion Sucties, AEC, S. AFRICA

Prot. M.A. Mellboro. Univ. of Natw, S. AFaICA

Prot. D.E. Kim, Pohang het. of Sai. 2 Tech., SO. KOAEA

Prof. C.I.E.MA.T, Fueion Division Libray, SPAIN

Dr. L Simnio, Univ. of UMEA, SWEDEN

Librery, Poyd inct of Technology, SWEDEN

Prof. H. Wrthelmeon, Chalmere Univ. of Tech., SWEDEN

Comse Phys. Dese Plasmas, Ecole Potylech. SWITZERLAND

Bibliotheck, Ince. Vocr Plawne-Fyeica, THE NETHERLANDS

Aage Prot. Dr. S. Cekir, Mudd East Tech. Univ., TURKEY

Dr. V.A. Chuthint,Sa. Aas. Inat Enctrophys.I Apparenus, USSR

Dr. D.D. Pyutov, Siberion Brench of Academy of Sa., USSR

Dr. GA. Eisenev, I.V. Kurchatov Inst, USSA

Librerion, The Uwr.SSA Acaderny of Sciencos, USSR

Dr. LM. Kowiathnyth, Inet. of Conerd Phycics, USSA

Kemionechungesedeos GmbH, Zentrabibliothek, W. GERMANY

Eibliotsele, Inet For Plasmatorechung. W. GERMANY

Prof. K. Schinder, Ruhr-Univereitu Bochum, W. GERMANY

Dr. F. Wogrex, (ASOEX), Max-Planck-Inetitut, W. GERMANY

Librevien, Max-Pbenck-Institut, W. GERMANY

Prot. R.K. Janew, Inst of Ptycics, YUcosLAVIA 

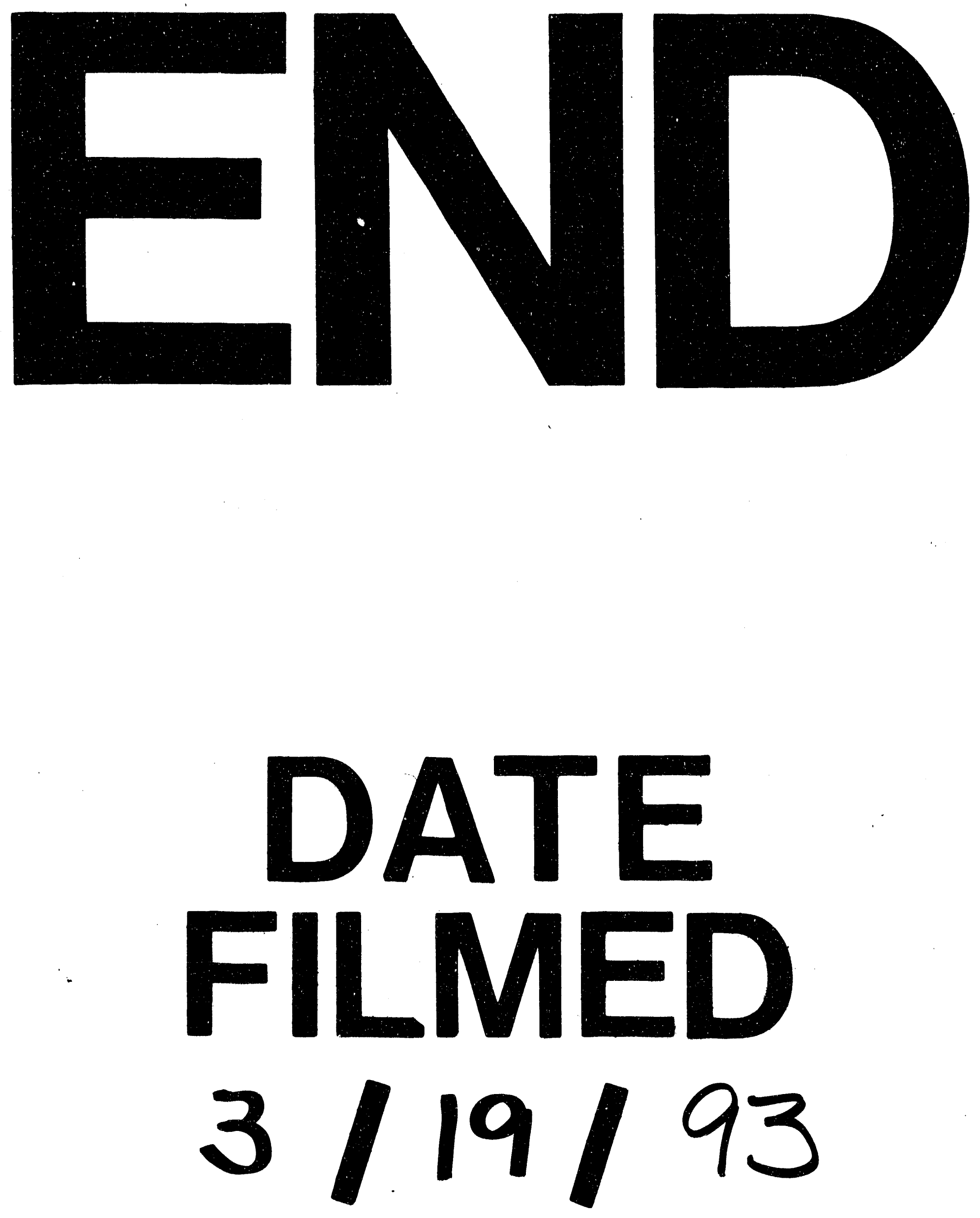

1 
